\title{
POLARIZATION CONTROL BY TERAHERTZ
}

METAMATERIALS

言

CONG LONGQING

$\stackrel{\sim}{\stackrel{0}{\sigma}}$

SCHOOL OF PHYSICAL AND MATHEMATICAL

SCIENCES 



\section{POLARIZATION CONTROL BY TERAHERTZ METAMATERIALS}

CONG LONGQING

School of Physical and Mathematical Sciences

A thesis submitted to the Nanyang Technological University in fulfillment of the requirement for the degree of Doctor of Philosophy 



\section{Acknowledgements}

The completion of this work requires the helps and supports from different sources. Here, I would like to thank all the sources that have supported and helped me on pursuing my $\mathrm{PhD}$ degree.

First and foremost, I would like to thank my supervisor Prof. Ranjan Singh for providing plenty of guidance on research topics, fruitful discussion on the proposal and results, unconditional financial and facility supports on the projects and the ample freedom to explore my own ideas.

I would also like to thank my co-supervisor Prof. Zhang Baile for his supporting and supervision.

I am sincerely grateful to my collaborators, Prof. Zhang Weili and Dr. Xu Ningning, for their helps on some measurements and the preparation of some samples.

I thank my colleague Mr. Yogesh Kumar Srivastava for his patient and timely supporting on preparation of samples.

I express my gratitude to Prof. Han Jiaguang and his group at Tianjin University for the supporting of facilities and valuable discussion;

I thank my collaborators Prof. Lee Chengkuo and Dr. Prakash Pitchappa for the supporting of MEMS samples, and Prof. Yang Hyunsoo and Dr. Wu Yang for their helps on reflection measurements.

I would also like to thank Nanyang Technological University for providing me the financial supporting on my $\mathrm{PhD}$ project. It was a great honor to work in the ultrafast terahertz 
photonics lab and center for disruptive photonic technologies with the well-equipped environment and state-of-art facilities.

I especially thank all of my colleagues at ultrafast terahertz photonics lab and center for disruptive photonic technologies, Mr. Manukumara Manjappa, Mr. Yogesh Kumar Srivastava, Mr. Han Song, Dr. Govind Dayal, and Mr. Manoj Gupta for their kind understanding and immense helps.

Finally, I sincerely thank my family for their unconditional supports of my decision on pursuing $\mathrm{PhD}$ degree, and my fiancée, Ms. Zhou Ye, for her understanding and supports.

Cong Longqing at SPMS building, Nanyang Technological University September 2016 


\section{Table of Content}

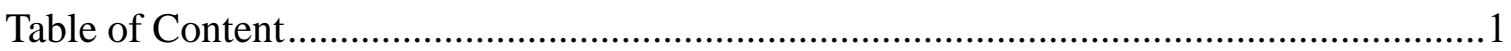

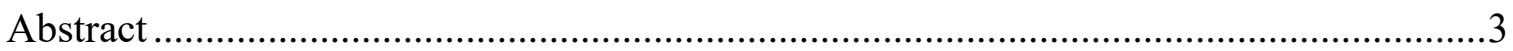

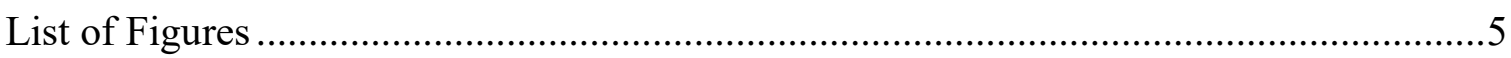

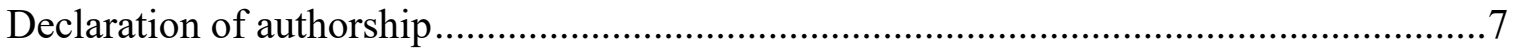

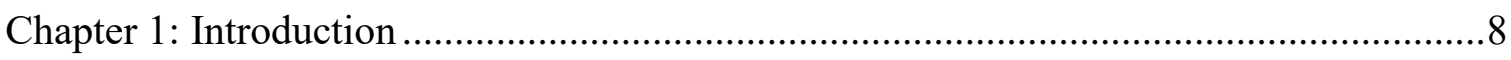

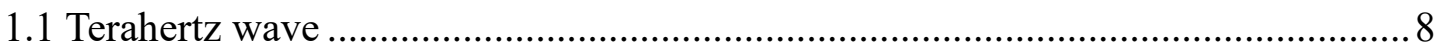

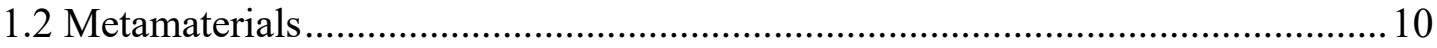

1.3 Polarization manipulation by metamaterials ..................................................... 12

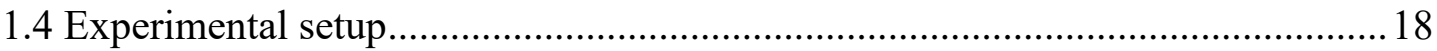

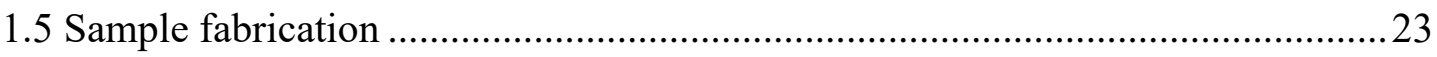

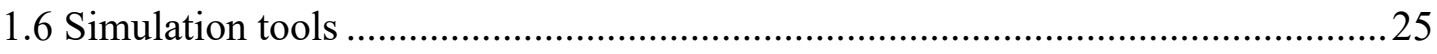

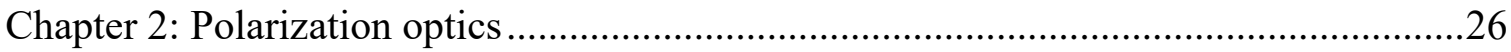

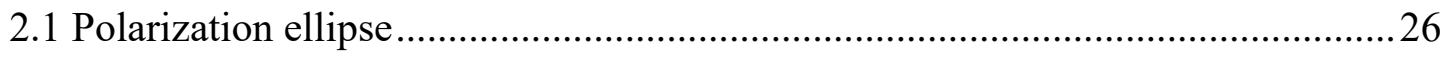

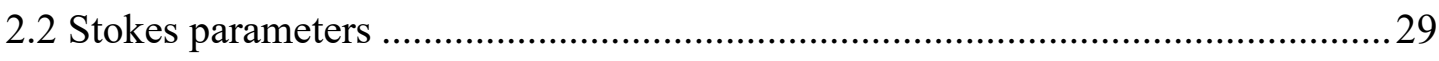

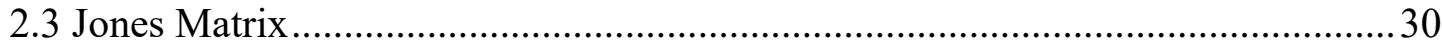

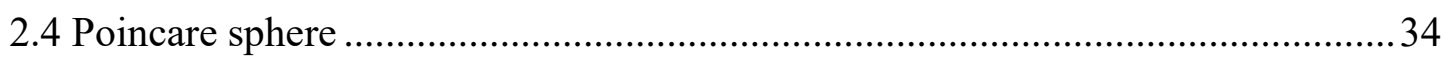

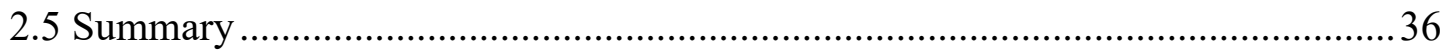

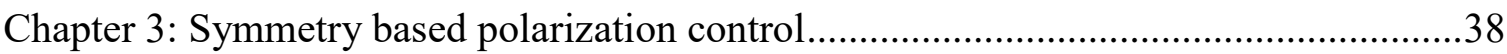

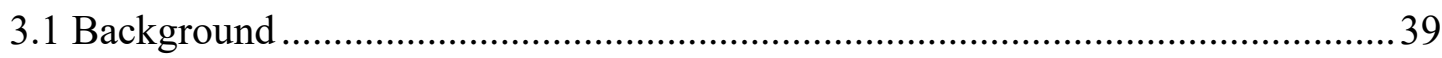

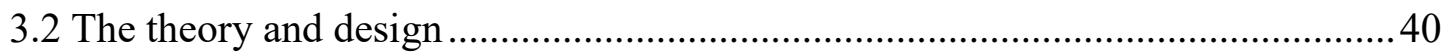

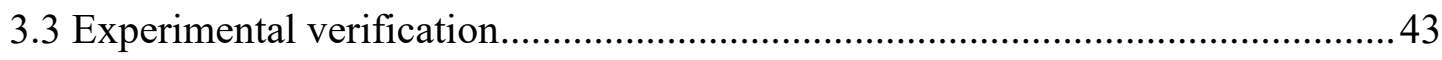

3.4 Explanation from electric field distributions .................................................. 46

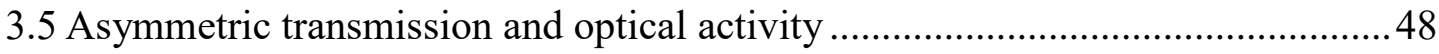

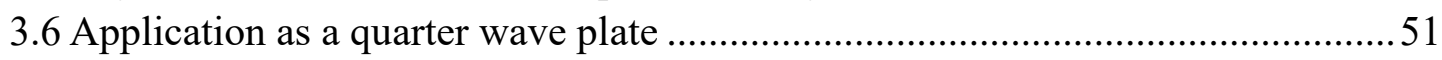

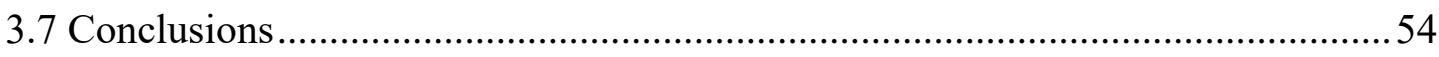

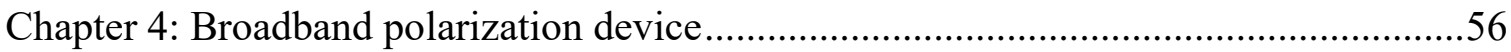

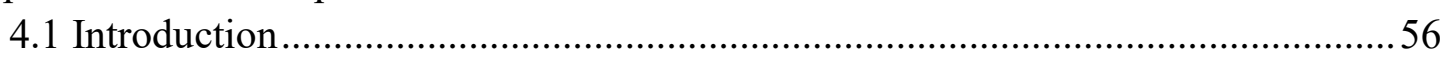

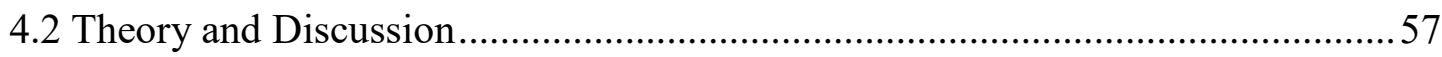

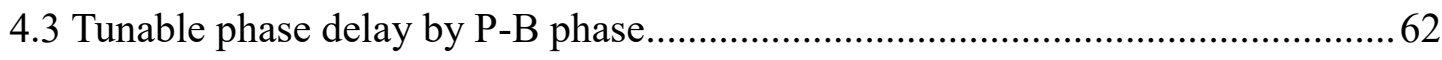

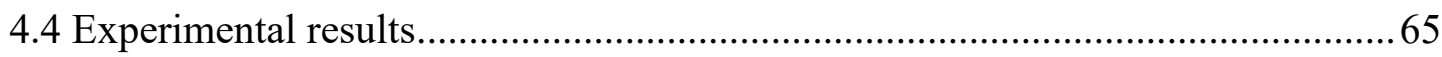

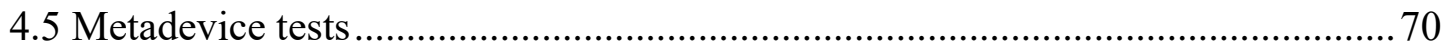

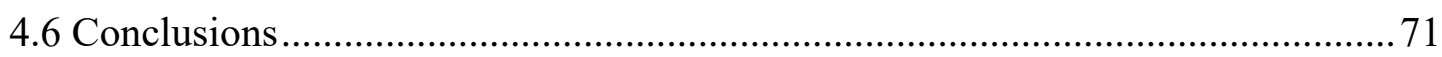

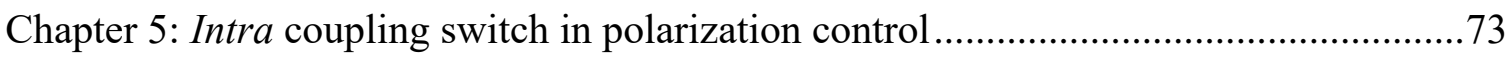

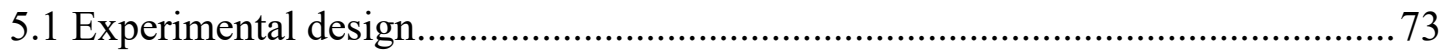

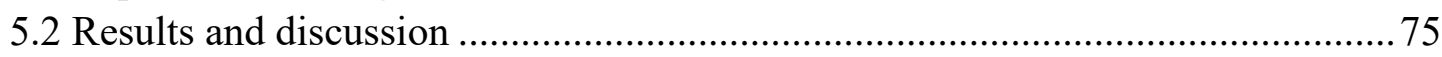

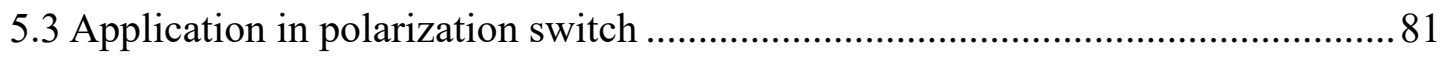

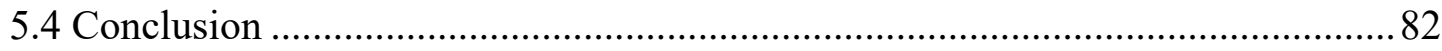

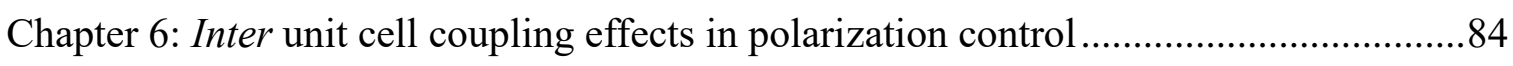

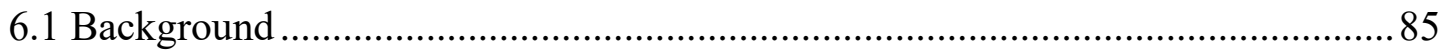

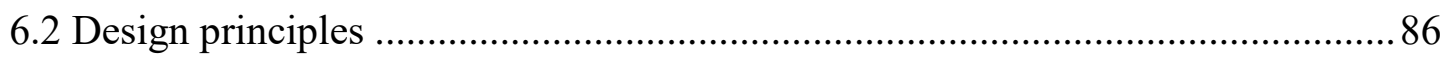

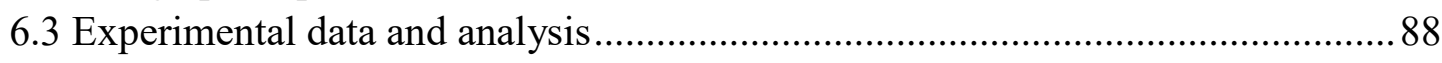

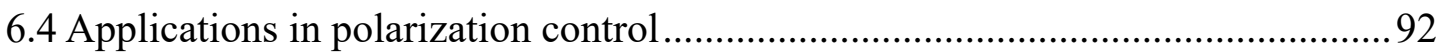




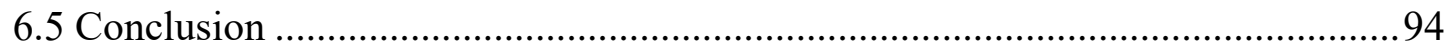

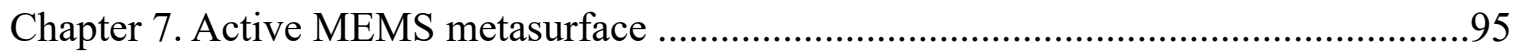

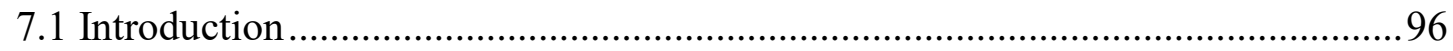

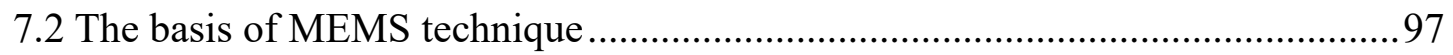

7.3 Disturbance theory and applications in MEMS metadevices .............................100

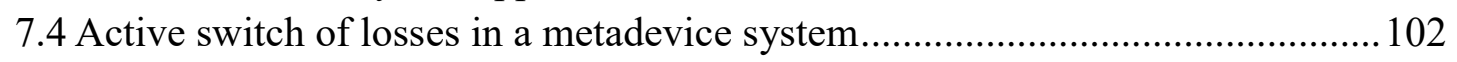

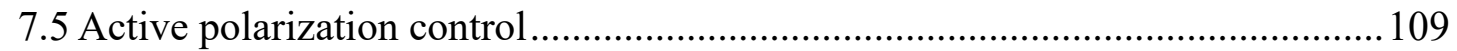

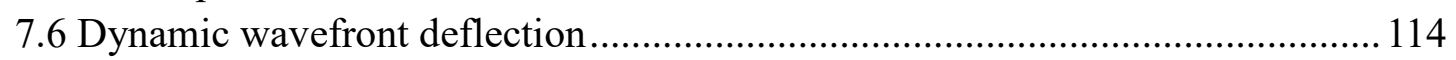

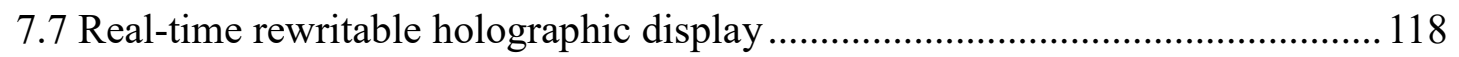

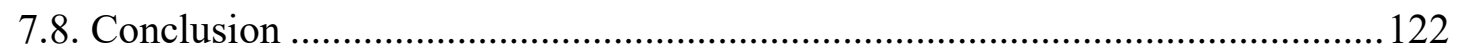

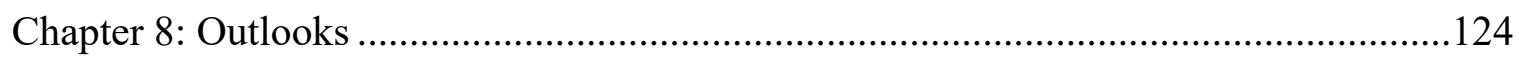

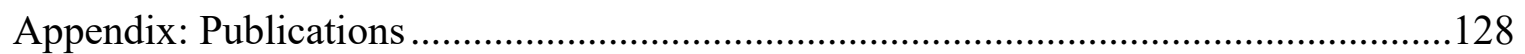

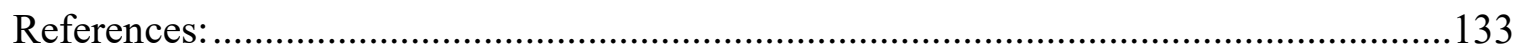




\section{Abstract}

Terahertz wave has emerged to be an extremely useful and important tool for applications in sensing, security check and space communications, however, the development is limited due to its weak interaction with the natural occurring materials. The artificially engineered metamaterials are able to provide an excellent platform to manipulate the properties of terahertz radiation. Among all the properties of light, polarization is one of the most important characterizations together with its intensity and wavelength. In this thesis, I will focus on the manipulation of polarization state of terahertz radiation using metamaterials from the aspect of applications as real polarizing devices. With the design freedom and the extraordinary optical properties of metamaterials, in this thesis I discussed the intrinsic symmetry dependence in the unit cell and coupling mechanism in the array for the highefficiency and broadband operation (Chapter 3, $5 \& 6$ ); and proposed an achromatic terahertz quarter wave plate and an active polarizing device using micro-electro-mechanical system (MEMS) technique (Chapter 4 \& 7).

This thesis is organized in such a way. In Chapter 1, I give a brief introduction of the fundamental knowledge for terahertz radiation and metamaterials, and discuss the progress of polarization control using metamaterials in detail. The experimental setup, sample fabrication and simulation methods are also presented in this Chapter.

In Chapter 2, I introduce the fundamental polarization optics and show the parameters to describe the polarization states of light. Polarization ellipse is the most straightforward 
method to describe the states, and Stokes parameters provide a measurable approach to experimentally present the states. More complex Jones Matrix connects the output and input ports by a $2 \times 2$ matrix that reveals the properties of the media interacting with the incident light. Finally, Poincare sphere is briefly introduced to describe the states.

In Chapter 3, polarization conversion behavior is discussed in terms of symmetry in the unit cell of the metamaterial array. A flexible metadevice is proposed in Chapter 4 that operates as a quarter wave plate in a broadband terahertz regime, and the phase response is modulated based on the P-B phase. Intra and inter unit cell coupling in a planar metamaterial always exists in the resonator array, and the effects originating from the coupling are discussed in Chapter 5 and 6 . In addition to the passive way to control the polarization states, I also introduce an active approach by combining the MEMS technique into metamaterial systems in Chapter 7. Polarization states are dynamically modulated by applying external voltage on the MEMS based metadevices. More polarization related applications are also presented using the MEMS based metadevices.

Finally, the outlook in Chapter 8 gives an overview of my future plans and the possible research directions related to polarization optics and metamaterials. 


\section{List of Figures}

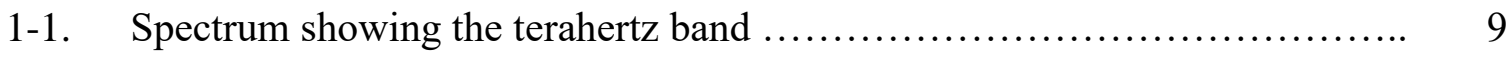

1-2. Metamaterial related pioneer works................................. 11

1-3. Chirality effects for polarization manipulation .......................... 13

1-4. Polarization control related works using metamaterials ................... 17

1-5. A typical $4-f$ setup for $\mathrm{THz}$ generation and detection with femtosecond pulses ....

1-6. Schemes of generation and detection of $\mathrm{THz}$ radiation by optical pulse

1-7. Schematic illustration for the measurements of Jones Matrix elements

2-1. Polarization ellipse.

2-2. Schematic diagram of stereographic projection for the polarization ellipse represented on a complex plane to a sphere surface.

3-1. Images of samples with broken of a certain symmetry by varying the gap positions of the double-gap split ring resonator array....

3-2. Experimental and simulated results of cross-polarized transmission signals at different symmetry configurations.

3-3. Underlying mechanism of cross-polarized component excitation ............ 47

3-4. Study of circularly polarized light transmission behaviors for $r$-SRR......... $\quad 50$

3-5. Performance of $r$-SRR to function as a quarter wave plate.................. 52

3-6. Measured transmission elements of Jones matrix ......................... 53

4-1. Transmission engineering of grating................................... 59

4-2. Approach of dispersion compensation with two-layer metasurfaces........... 61

4-3. Measured and simulated transmission amplitude, phase response and ellipticity of two orthogonal polarization components............................. 62

4-4. P-B phase interpretation and application ............................. 64

4-5. Fabricated sample and measured transmission response ................... 66

4-6. Investigation of the metadevice performance in details................... 68

4-7. Tests of the metadevice on the flexibility and stability performance......... 70

5-1. Experimental design ............................................ $\quad 74$

5-2. Measured and simulated transmission spectra of MM1 and MM2 ......... 76

5-3. Cross-polarized spectra and understanding of angle-dependent intra coupling switch ........................................................ 78

5-4. Polarization modulation performance of the metasurface .................. 81

6-1. Resonator excited with LC resonance at different lattices ................... 84

6-2. $\quad$ Design principle to study the inter coupling effects .................... 87

6-3. Far-field transmission spectra of the two meta-molecular arrays.............. 88

6-4. Detailed amplitude transmission spectra with varied $p_{y} \ldots \ldots \ldots \ldots \ldots \ldots \ldots . . \quad 90$

6-5. Potential applications in polarization control........................... 92

7-1. Fundamental resonance profile of the simulated deformation cantilevers.... 99

7-2. Anisotropic metadevice design..................................... 101 
7-3. Metal-insulator-metal (MIM) system described using the coupled-mode theory

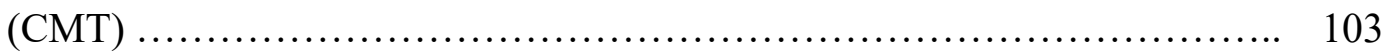

7-4. Active switch of operation state by a MEMS metadevice................. 105

7-5. Experimental and simulated results of active switch in operation state........ 107

7-6. Active polarization control performance of the MEMS metadevice ........... 111

7-7. Proposed scheme of the dynamic reflection wavefront deflection ............... 115

7-8. Proposal of a real-time holographic display .............................. 119 


\section{Declaration of authorship}

I, Cong Longqing, declare that the thesis entitled "Polarization control in terahertz metamaterials" and the work presented in the thesis are proposed by my own as the results of the original research. I would like to confirm that:

- This work was done entirely in the period of candidature for a research $\mathrm{PhD}$ at Nanyang Technological University;

- It has been clearly stated where any part of the thesis has previously submitted for any other qualification;

- It has been clearly attributed with the sources given where I consulted others' published works;

- I have acknowledged all the helps from different sources;

- I have clearly stated what was done by others and what was contributed by myself for the jointly performed works;

- I have listed the published papers in the journals or conferences in the Appendix.

Signed: Cong Longqing

Date: 20-Jan-2017 


\section{Chapter 1: Introduction}

\subsection{Terahertz wave}

Terahertz (THz) is a commonly used term to refer to the band of frequency lying between the microwave and infrared regions in the electromagnetic spectrum. Despite of the invisibility of the THz radiation, we can feel the warmth of it since it is also in the farinfrared region. Although the $\mathrm{THz}$ radiation exists everywhere in space, the technical difficulties in developing high-efficient $\mathrm{THz}$ sources and detectors lead to this frequency region least explored. In recently two decades, more and more research efforts have been devoted into diminishing the so-call "THz gap" from two approaches: optical techniques from high frequency side by optical rectification and difference frequency generation; and microwave techniques from the low frequency side by frequency multiplication of microwaves. In practice, different units are used to describe the $\mathrm{THz}$ radiation where the frequently used units at $1 \mathrm{THz}$ are listed below:

- Frequency: $f=1 \mathrm{THz}=1000 \mathrm{GHz}$

- Wavelength: $\lambda=c / f=300 \mu \mathrm{m}$

- Period: $\mathrm{T}=1 / f=1 \mathrm{ps}$

- Angular frequency: $\omega=2 \pi f=6.28 \mathrm{THz}$

- Photon energy: $h v=4.14 \mathrm{meV}$

where $c$ is the vacuum speed of light and $h$ is the Plank's constant. Here the THz band is usually defined between 0.1 to $10 \mathrm{THz}$ as illustrated in Figure 1-1 which connects the highest radio frequency band and far-IR band. 


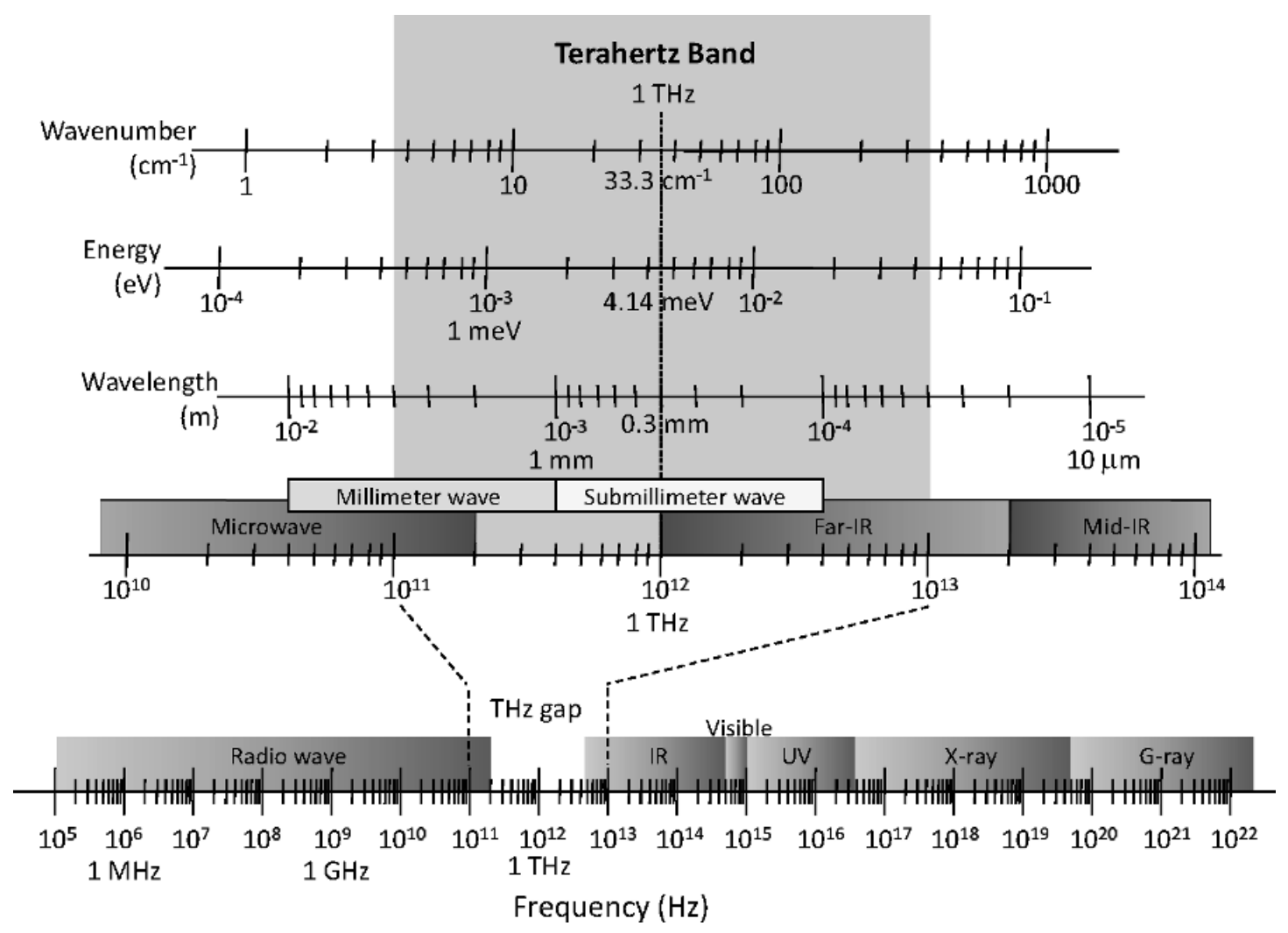

Figure 1-1. Spectrum showing the terahertz band. ${ }^{1}$

Innumerable spectral features of fundamental physical processes gather in $\mathrm{THz}$ region such as vibrational motion of organic compounds, lattice vibrations in solids, energy gaps in superconductors, intraband transitions in semiconductors and rotational transitions of molecules. Each molecular species would have distinctive spectral line shapes that provide the crucial information of the microscopic mechanisms of molecular collisions for the identification of an unknown specimen. The Earths' atmosphere and interstellar medium are being monitored by using the high-resolution $\mathrm{THz}$ spectroscopy. In biology, the molecular dynamics associating with vibrational motions and inter molecular interactions can be monitored from the spectral signatures by $\mathrm{THz}$ spectroscopy. The detection of explosives and illicit drugs, testing of pharmaceutical products and protein conformation, 
etc, are also feasible by $\mathrm{THz}$ spectroscopy. For condensed matter in natural world, three types are grouped according to the $\mathrm{THz}$ optical properties including water, metal and dielectric. ${ }^{1}$ In THz region, water is highly absorptive due to the strong polarity whose spectrum reveals distinctive features; metals would reflect all the radiation with the high conductivity; and dielectrics such as clothes, wood and ceramics that are opaque at visible range are usually transparent in the $\mathrm{THz}$ band. Many imaging applications ${ }^{2}$ ranging from basic scientific missions to commercial projects are possible with these stark contrasts of different media in $\mathrm{THz}$ band such as nondestructive testing for sealed packages, security applications and medical applications. THz radiation can penetrate through the package that is usually made of dielectrics and detect the inclusions inside. It's easy to differentiate the hydrated substances from dried ones due to the high absorption $\mathrm{THz}$ spectrum of water. Metal objects are identified with the high reflectivity in order to detect weapons. In medical applications, the sensitivity of water in $\mathrm{THz}$ spectrum would provide the useful tool to inspect the water content that is crucial for the quality of drugs and biological systems.

\subsection{Metamaterials}

Metamaterials are the assemblies of several elements arranged in a repeating pattern with the unit cell at scale smaller than the operating wavelength. Through precisely engineering the size, shape, geometry, orientation and arrangement of the unit cells, metamaterials would enable the manipulation of electromagnetic waves by absorbing, ${ }^{3-5}$ enhancing, ${ }^{6}$ or bending waves ${ }^{7}$ to realize properties that are beyond the capability of natural materials as shown in Figure 1-2. The Metamaterials have the ability to realize the phenomena that are 
not capable by natural materials such as negative refractive index media, ${ }^{8-10}$ enhanced lightmatter interaction, ${ }^{11-14}$ extraordinary transmission ${ }^{6}$ and designer planar optical devices ${ }^{15,16}$ with the artificially designed unit cells.
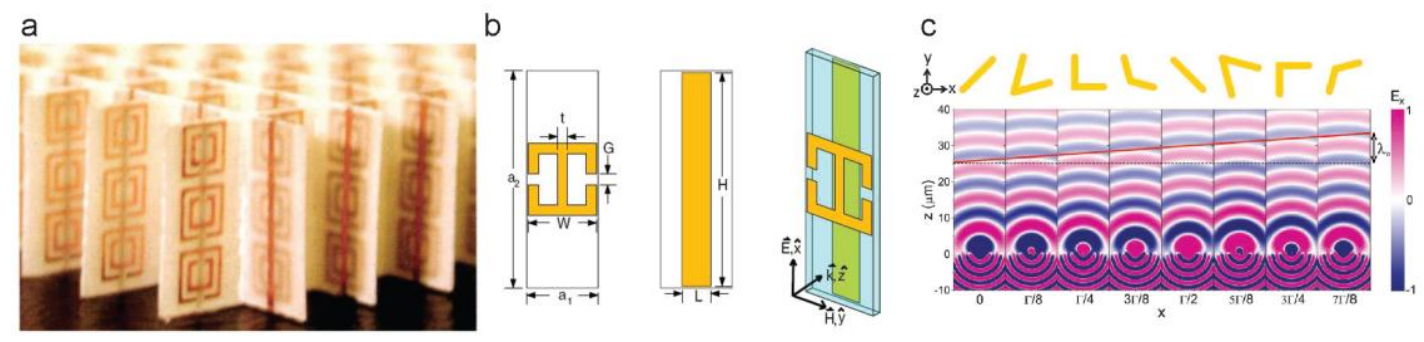

Figure 1-2. Metamaterial related pioneer works. (a) The first experimental demonstration of negative refractive index. (b) The first metamaterial perfect absorber design. (c) The first work in metasurface with engineered phase gradient.

The theory of negative refractive index materials was initially proposed by Victor Veselago in 1967 who stated the phase velocity of light anti-parallel to the direction of Poynting vector propagating in a such kind of medium. ${ }^{17}$ John Pendry proposed a practical way by left-handed metamaterials in order to convey energy against the phase velocity using the metallic wires that provide the negative permittivity and split ring resonators that provide the negative permeability. ${ }^{18}$ The first experimental demonstration of negative index metamaterials was reported by Smith et al. with design as shown in Figure 1-2(a). ${ }^{8}$ Metamaterial perfect absorbers ${ }^{5}$ are useful for photodetection and solar photovoltaic applications, and the concept and experimental design were first proposed by N. Landy et al with the design shown in Figure 1-2(b). Most recently, planar metamaterials with engineered phase gradient ${ }^{7}$ was proposed by N. Yu et al as shown in Figure 1-2(c) that 
opens up the study of metasurface for planar optics in applications of flat lenses, ${ }^{19}, 20$ holograms, ${ }^{21,22}$ cloaking $^{23}$ and encoded metasurfaces. ${ }^{24,25}$

\subsection{Polarization manipulation by metamaterials}

The superiority of artificially designed metamaterials reflects on the realization of phenomena that natural materials cannot obtain, and the extraordinary manipulation of polarization has also been discussed and investigated in metamaterial community. For example, optical activity is a commonly used parameter to measure the chirality of materials, and it describes the rotation of the incident linearly polarized plane by the materials. Optical activity is normally observed in gases or solutions with chiral molecules such as sugars, proteins and liquid crystals, however, the rotation is usually extremely weak from these natural materials, and thus is very difficult to be experimentally observed. One of the earliest discussion was proposed by Sir John Pendry in realizing the negative refractive index with a chiral route theoretically ${ }^{10}$ and subsequently experimentally verified by Shuang Zhang. ${ }^{26}$ Several extraordinary effects have been reported to be obtained by metamaterials as shown in Figure 1-3(a), such as the strongly enhanced optical activity using the artificially designed chiral meta-molecules ${ }^{27}$ and the extrinsic optical activity by planar meta-molecule without intrinsic chirality in the unit cell itself through tilting the incidence vector. ${ }^{28}$ Another approach to rotate the polarization plane is through the Faraday effect that requires applying external static magnetic field, however, this is a magnetooptical phenomenon but not due to the optical activity of chiral molecules. The polarization plane rotation due to Faraday effect is also extremely weak with the natural magnetic 
materials, and it was reported to be enhanced by almost an order by integrating the magnetic materials with plasmonic meta-molecules as shown in Figure 1-3(b). ${ }^{29}$ The handness of chirality in various natural materials has been discovered to be switched by external stimuli such as organic molecules. ${ }^{30,31}$ However, the substantial weak effect of optical activity in natural materials limits the contrast of chirality switching. The enhanced contrast of chirality switching was firstly demonstrated in terahertz region by the optical pump method with a three-dimensional metamaterial design as shown in Figure 1-3(c). ${ }^{32}$ Most recently, another giant chirality switching approach was reported by a MEMS based spiral metamaterial in terahertz regime, and the switching is realized by the forces from the air pressure difference. ${ }^{33}$

a
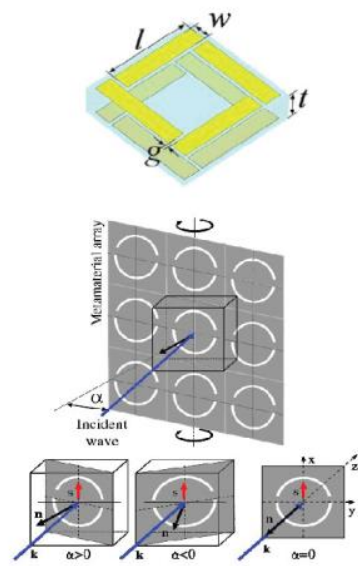

d

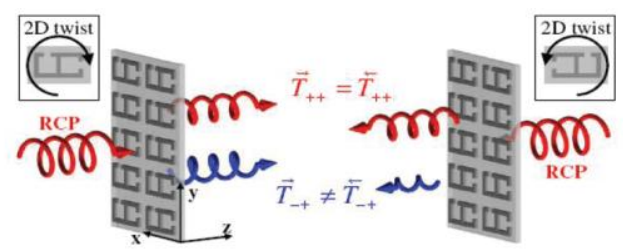

b

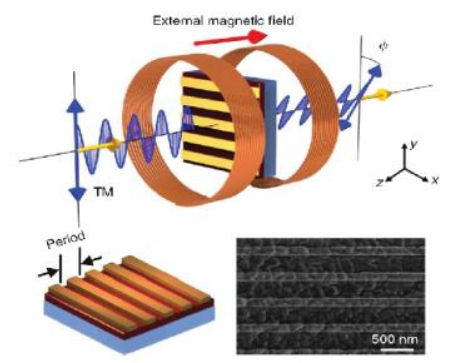

C

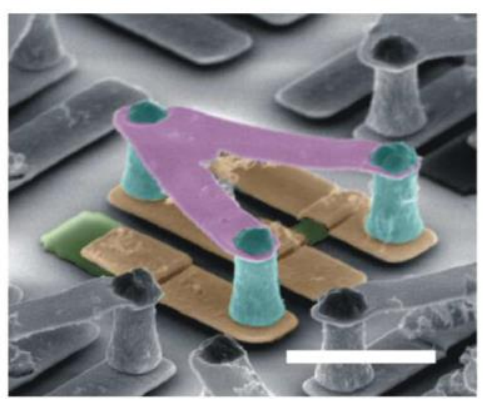

e

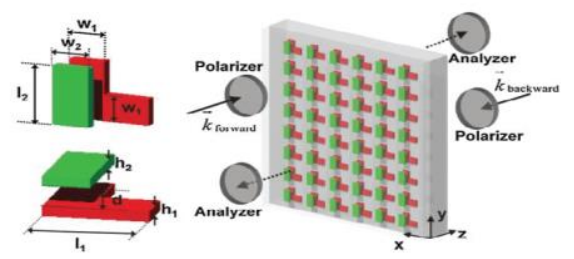

Figure 1-3. Chirality effects for polarization manipulation. (a) Optical activity by intrinsic chirality and extrinsic chirality. (b) Faraday rotation effect enhancement by 
integrating with plasmonic meta-molecules. (c) Active switching of intrinsic chirality by optical pump. (d) Asymmetric transmission of circularly polarized light with planar chiral meta-molecules and (e) asymmetric transmission of linearly polarized light by breaking the symmetry of meta-molecules along wave propagating direction.

An interesting phenomenon related to the planar chiral metamaterials was discovered that exhibited the asymmetric transmission for cross-polarized component of circularly polarized light when light transmitted from front to back and from back to front with the meta-molecules as illustrated in Figure 1-3(d). ${ }^{34,35}$ This phenomenon occurs in the lossy anisotropic planar metamaterials whose eigen states are two co-rotating elliptical polarizations, and it is different from the gyrotropy in the bulk chiral materials and the Faraday effect. For such a planar metamaterial with extrinsic chirality, asymmetric transmission is only applicable for circularly polarized light but not linearly polarized one. In order to obtain the asymmetric transmission of linearly polarized light, the symmetry of meta-molecule along the propagation direction of light (wave vector) requires to be broken and a three dimensional or multilayer metamaterials are necessary in order to obtain the distinct linearly cross-polarized components induced by the metamaterials, i.e. $\tilde{t}_{x y} \neq \tilde{t}_{y x} \cdot{ }^{36}$ The first demonstration of asymmetric transmission for linearly polarized light with doublelayer metamaterial is as illustrated in Figure 1-3(e).

The most important applications of polarization control are for devices that can be applied as components in optical setups, communication systems and displays. Nowadays, the 
commonly used polarization components are wire-grid polarizers as a linear polarizer or analyzer in THz systems, and a birefringent crystal based wave plate such as a quarter wave plate for generation of circularly polarized light and a half wave plate for orthogonal polarization rotation in optics systems. It is commonly known, by employing the birefringence of natural materials, the operation will be limited at one single specific wavelength while cutting the crystal with a designated thickness. This intrinsic issue due to the frequency dispersion in the natural materials extensively restrict the applications. The solutions for broadband operation to obtain circularly or linearly polarized light were discovered by using metamaterials. The first demonstration of the broadband circular polarizer was by the three-dimensional gold helix meta-molecules that realized the strong circular dichroism in a broadband frequency range with two pitches of helixes as shown in Figure 1-4(a). ${ }^{11}$ Although this design reveals excellent performance of circular dichroism, the three-dimensional nano-helix requires extremely complicated and rigid fabrication technique. One alternative is by stacking several layers of metasurfaces in order to avoid the three-dimensional structures as shown in Figure 1-4(b) where the circular dichroism is obtained by the relative rotation of the resonator in the longitudinally neighboring metasurfaces. The performance of circular dichroism and operation bandwidth are both improved by stacking more layers of metasurfaces, however, the transmission efficiency will be reduced thereupon, and the alignment of the metasurfaces would be another challenge in fabrication. In this thesis, I will propose a solution for the high-efficient and broadband generation of circularly polarized light with only two layers of metasurface in

\section{Chapter 4.}


For a linear polarizer, the smartest approach is by using the wire grid grating that shows the broadband and high-efficient performance. The commercially available polarizer usually uses tungsten as the material of wires, however, it is very fragile and requires the substrate as a support. One substitution was proposed as the wires for the linear polarizer that is shown in Figure 1-4(c) where carbon nanotube was employed in a free-standing fashion and it also revealed excellent performance as a linear polarizer. ${ }^{37}$ Another component for linear polarization control is the polarization rotator. Orthogonal polarization rotation is achievable by a half wave plate in a single wavelength for the traditional polarizing devices. The operation bandwidth is also demonstrated to be broadened using a metameterial design with one feasible way as illustrated in Figure 1-4(d) in reflection mode using the high-index dielectric resonator with reduced losses. 
a

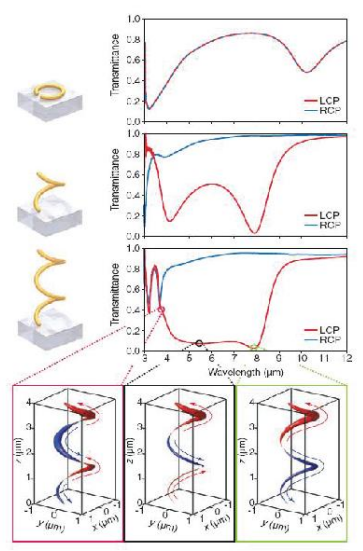

C

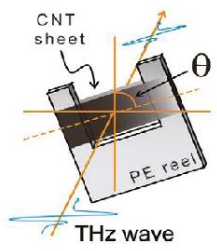

e
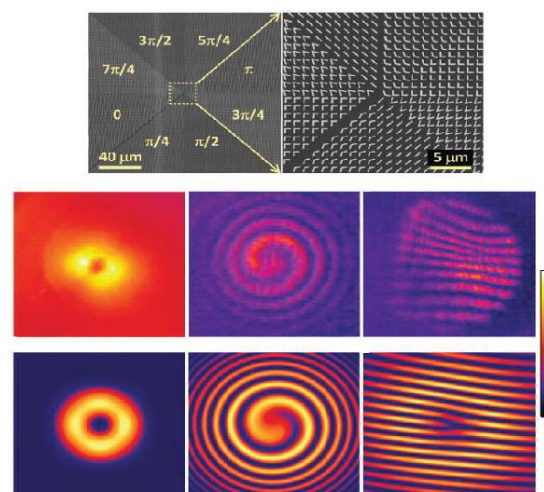

b
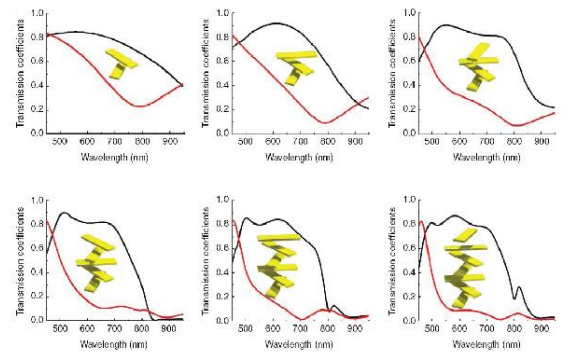

d
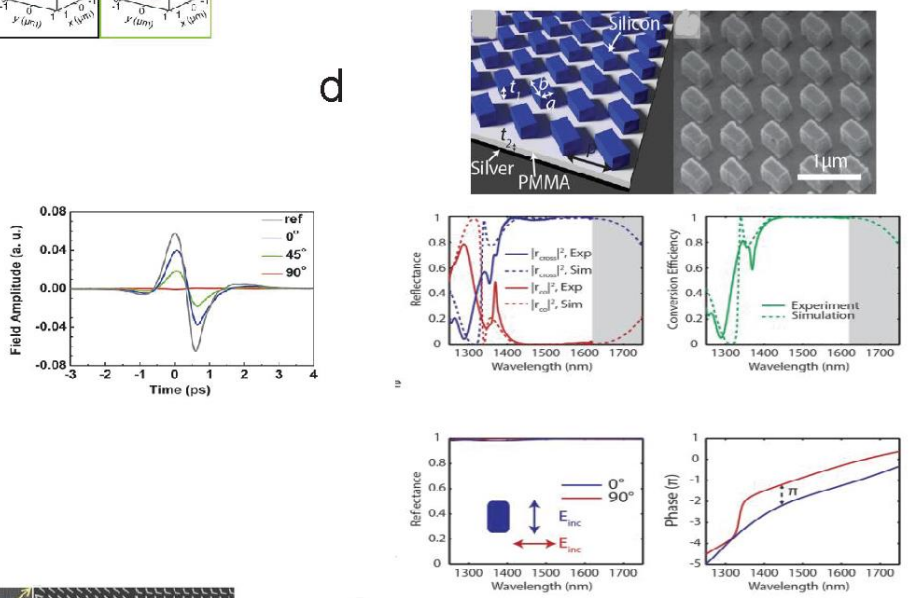

f
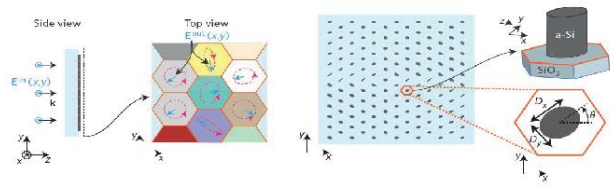

Figure 1-4. Polarization control related works using metamaterials. (a) 3D gold helix metamaterials as a broadband circular polarizer. (b) The multilayer metasurfaces with rotated orientation of resonators as a broadband circular polarizer. (c) A reel-wound broadband linear polarizer using carbon nanotube for terahertz wave. (d) Broadband polarization rotator operating in reflection mode. (e) Optical vortex generation by metasurface. (f) A complete control of polarization using dielectric metasurfaces. 
The recently developed planar metasurface concept in metamaterial community enables the generation and detection of optical vortex as shown in Figure 1-4(e) where the local phase responses are tailored by carefully designing the meta-molecules, and the unit cell of the metamaterials are no longer repeated in the whole array but in a more complicated supercell or arranged in a designated fashion following the functional equations. A complete control of phase and polarization in the subwavelength resolution has been reported by the dielectric metasurfaces for applications in focusing, imaging and vector beam generations with high efficiency as shown in Figure 1-4(f). ${ }^{38}$

\subsection{Experimental setup}

Terahertz time-domain spectroscopy (THz-TDS) system was employed to characterize the response of metamaterial samples in all of my experiments. Most transmission measurements were done using an 8-f antenna based terahertz system in this thesis with the schematic setup shown in Figure 1-5. For simplicity, we present a $4-f$ setup in the illustration where two parabolic mirrors are employed to focus the $\mathrm{THz}$ beam, and the parallel beam will interact with the samples. In practice, an $8-f$ system is more commonly employed with four parabolic mirrors that focus the $\mathrm{THz}$ beam at a smaller point at which the sample locates in order to enhance the light-matter interaction. Several approaches have been reported to generate and detect $\mathrm{THz}$ radiation such as photoconductive antennas, optical rectification with nonlinear crystals and air plasma generated by high-power laser pulse. The most efficient and convenient way is by photoconductive antenna method which is also 
the approach employed in my experiments. We will then mainly introduce and discuss the basis of photoconductive switching antenna in this section. For more detailed information of different $\mathrm{THz}$ radiation generation and detection methods, please refer to Ref. $1 .^{1}$

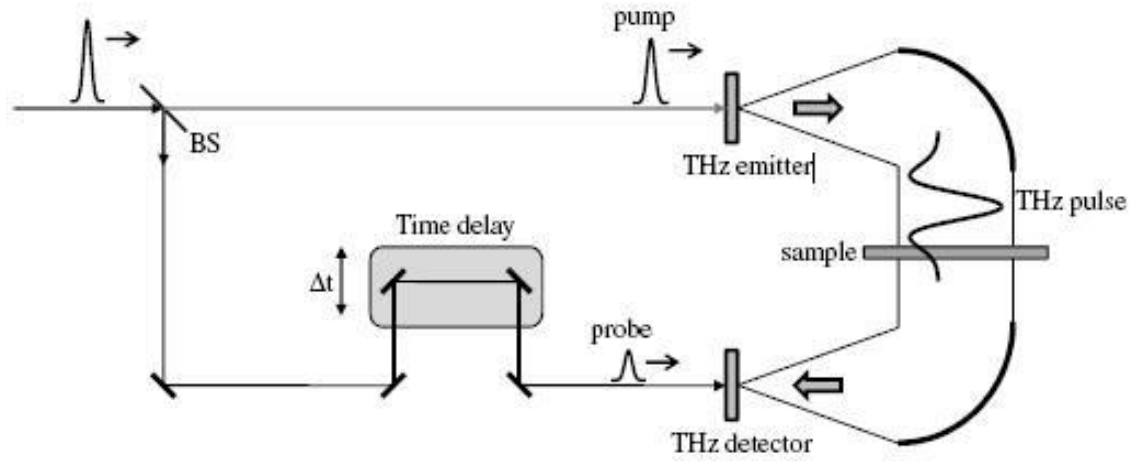

Figure 1-5. A typical 4- $f$ setup for THz generation and detection with femtosecond pulses. ${ }^{1}$

When the photoconductive antenna, that is in fact an electrical switch, is exposed to light, the electrical conductivity of semiconductors increases leading to an increase of free carriers generated by photons with energy higher than the bandgap. The switching behavior is required to occur in the subpicosecond time scale in order to generate and detect $\mathrm{THz}$ radiation where the switch-on time depends on the laser pulse duration and the switch-off time is determined by the relaxation time of photoexcited carriers in the substrate of antenna. Therefore, the semiconductor substrate requires a short lifetime, and the higher carrier mobility and breakdown voltage also help to improve the $\mathrm{THz}$ radiation quality. Several materials have been reported for THz applications such as low-temperature grown gallium arsenide (LT-GaAs), amorphous silicon, chromium-doped gallium arsenide (Cr-GaAs), radiation-damaged silicon-on-sapphire (RD-SOS) and indium phosphide (InP) where LT- 
GaAs and RD-SOS are the most commonly used ones. Here in this thesis, we used LTGaAs antenna in the experiments. Molecular beam epitaxy (MBE) was used to grow the LT-GaAs in low temperature of $\sim 200^{\circ} \mathrm{C}$ followed by a rapid thermal annealing. The shortest lifetime is achieved at the grown temperature around $200^{\circ} \mathrm{C}$ that is $\sim 0.2 \mathrm{ps}$ and the effective carrier mobilities are $\sim 200-400 \mathrm{~cm}^{2} / \mathrm{V} \cdot \mathrm{s}$.

a

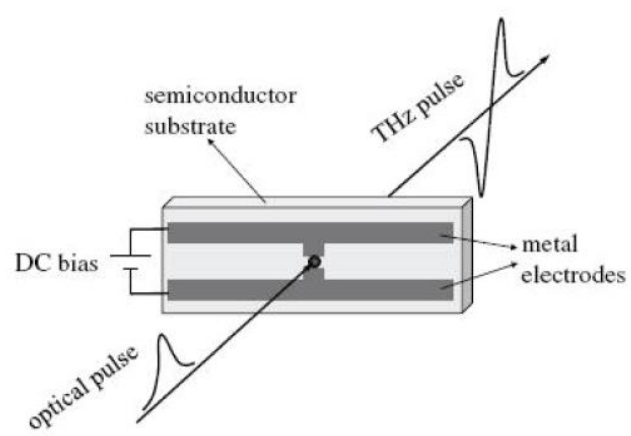

b Detection

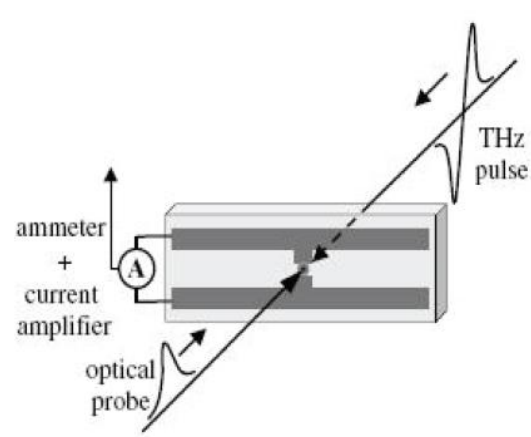

Figure 1-6. Schemes of generation and detection of $\mathrm{THz}$ radiation by optical pulse. ${ }^{1}$

With a pattern of metal electrodes as shown in Figure 1-6(a), external DC bias can be applied between the electrodes that accelerates the free carriers generated by the femtosecond laser pulse $(\lambda \sim 800 \mathrm{~nm})$. On the time scale of carrier lifetimes, the density of accelerated free carriers declines primarily by trapping in defect sites which forms the impulse current radiating the subpicosecond pulses of electromagnetic waves. The detection of $\mathrm{THz}$ radiation is almost identical to that of $\mathrm{THz}$ generation. As shown in Figure 1-6(b), the photocarriers injected by the optical probe pulse will be induced a current in the gap of electrodes without external bias applied. This detection technique is time-resolved and thus it requires the lifetime of photocurrent carriers much shorter than the $\mathrm{THz}$ pulse 
duration. The photocurrent intensity is proportional to the $\mathrm{THz}$ pulse amplitude and thus the THz waveform is mapped by recording the photocurrent in the time domain at varied time delay between the pump and probe beam. In practice, a current amplifier is required to enhance the sub-nanoamp current to the measurable voltages and a lock-in amplifier synchronized with an optical chopper is required to improve the signal-to-noise ratio.

The home-build free-space laser pump and probe system is only available for the transmission measurements. For the reflection measurements that is discussed in $\underline{\text { Chapter }}$ 7, a commercial available fiber based THz-TDS system (TeraView, TPS Spectra 3000) was used whose antenna is flexible to be configured for transmission and reflection measurements. In all the measurements, dry nitrogen or dry air were inflated into the $\mathrm{THz}$ beam path space in order to avoid the influence of vapour. With the time-domain waveform of samples and references (signals from silicon substrate of sample or dry air for transmission experiments, and an aluminum plate for reflection experiments), Fourier transform was then used to retrieve the frequency domain spectra with both phase and amplitude information. The amplitude and phase spectra are normalized by $\tilde{t}(\omega)=\tilde{t}^{\operatorname{Sam}}(\omega) / \tilde{t}^{\text {Ref }}(\omega)$ where $\tilde{t}^{\text {Sam }}(\omega)$ is the spectra of samples and $\tilde{t}^{\text {Ref }}(\omega)$ is the spectra of references. 


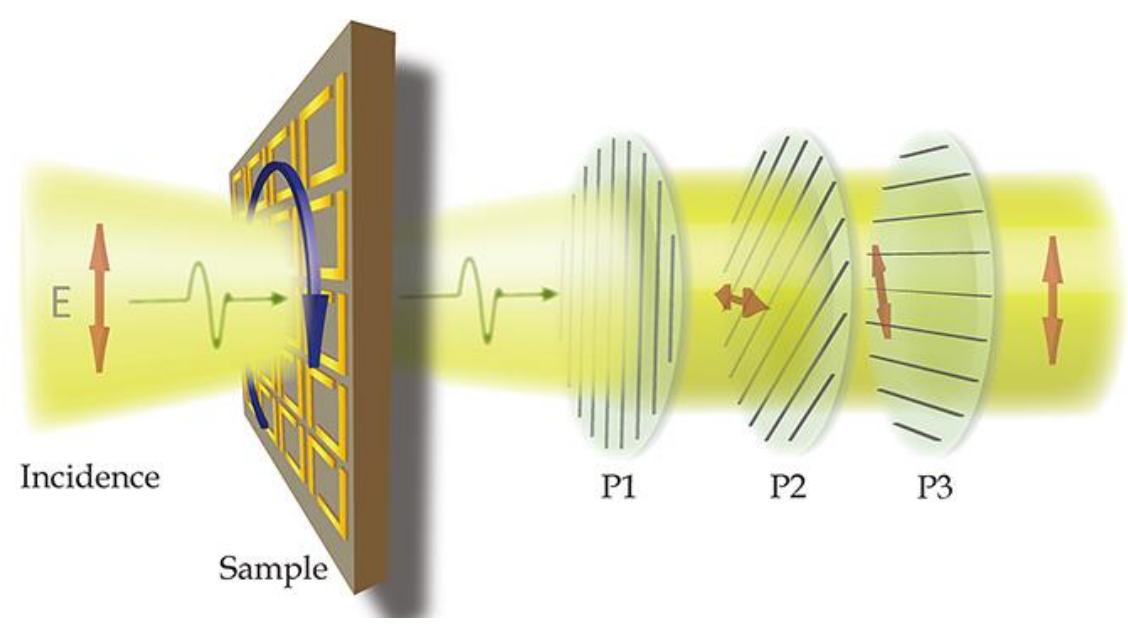

Figure 1-7. Schematic illustration for the measurements of Jones Matrix elements.

The photoconductive antennas are polarization sensitive, and in order to measure all the four elements $\left(\tilde{t}_{x x}, \tilde{t}_{y x}, \tilde{t}_{x y}\right.$ and $\left.\tilde{t}_{y y}\right)$ of Jones Matrix (details can be found in Chapter 2), the wire grid polarizers are needed to coherently obtain them with arrangement as shown in Figure 1-7. Three polarizers are inserted after samples that are named as P1, P2 and P3 with $\mathrm{P} 1$ as an analyzer, and $\mathrm{P} 2$ and $\mathrm{P} 3$ together as the rotator. In a system where the transmitter emits linearly polarized radiation along $y$-direction as show in the graph and the receiver is only sensitive to the components along $y$-direction, $\mathrm{P} 1$ functions to block all the co-polarized signals ( $y$-polarized) with orientation of grating along the $y$-direction and thus only the cross-polarized ( $x$-polarized) component transmits. However, the cross-polarized component polarized along $x$-direction is not measurable by the receiver so that $\mathrm{P} 2$ and $\mathrm{P} 3$ combine to rotate the transmitting component to polarize along $y$-direction that is measurable by the receiver. In this way, the cross-polarized component $\tilde{t}_{x y}$ is measured. For the other cross-polarized element $\tilde{t}_{y x}$, we just simply rotate the sample by $180^{\circ}$ and measure again. We note that the rotation of sample will introduce an extra $\pi$ phase change 
which will be removed in subsequent data processing. For the co-polarized elements, we can coherently measure them by the same way through rotating $\mathrm{P} 1$ by $90^{\circ}$.

\subsection{Sample fabrication}

For the normal planar metamaterials usually consisting of metal unit cells and highresistivity silicon substrate, the conventional photolithography and thermal metallization were used for the fabrication. For the photolithography process, the positive photoresist was first spun with thickness of around $1.5 \mu \mathrm{m}$ by the $3000 \mathrm{rpm}$ speed for $30 \mathrm{~s}$. The wafer with photoresist was then soft baked in thermal oven at $115^{\circ} \mathrm{C}$ for 15 mins in order to evaporate the solvent. After the preparation of the wafer, the exposure was ready to start by combining the mask with predesigned pattern and UV light transmitted through the mask will interact with the photoresist. We can then start the post exposure bake at $115^{\circ} \mathrm{C}$ for 5 mins followed by the development which will dissolve the exposed photoresist and leave the complimentary photoresist pattern on the wafer. Another step of hard bake was required to evaporate the development solution and solidify the photoresist pattern. The photolithography part has finished with the predesigned pattern on the substrate. The sample will be finalized by the next step of metallization using thermal evaporation, and metal will directly deposits on the substrate at the location without photoresist that forms the desired patter and the remaining part of metal that deposits on the photoresist will be lifted off in the final step by dissolving the photoresist in acetone.

For the MEMS metamaterials introduced in Chapter 7, a CMOS compatible process was 
used as described below. First, we need to clean a silicon wafer as the substrate for the sputtering deposition of around $1 \mu \mathrm{m}$ thick aluminum reflector layer (only for the metalinsulator-metal sandwich structure). After this, we start the deposition of a $5 \mu \mathrm{m}$ thick silicon oxide $\left(\mathrm{SiO}_{2}\right)$ spacer using plasma enhanced chemical vapor deposition (PECVD) method. Following this, a $100 \mathrm{~nm}$ aluminum oxide $\left(\mathrm{Al}_{2} \mathrm{O}_{3}\right)$ layer was deposited using atomic layer deposition (ALD) process that is for preventing the etching of spacer $\mathrm{SiO}_{2}$ during the release process. On top of this layer, we deposited a $100 \mathrm{~nm}$ thick $\mathrm{SiO}_{2}$ sacrificial layer using PECVD method.

Second, we move to pattern the anchors and cantilevers structures by the conventional photolithography process. With the predesigned pattern, the sacrificial $\mathrm{SiO}_{2}$ parts for anchor regions were first dry etched. After this, ALD process was used to deposit a $50 \mathrm{~nm}$ thick $\mathrm{Al}_{2} \mathrm{O}_{3}$ layer, followed by the sputter deposition process of $500 \mathrm{~nm}$ thick $\mathrm{Al}$. Note that the bimorph layers $\left(\mathrm{Al} / \mathrm{Al}_{2} \mathrm{O}_{3}\right)$ are in physical contact with bottom $\mathrm{Al}_{2} \mathrm{O}_{3}$ layer at the anchor region, and in the remaining part of the wafer, it is on top of $\mathrm{SiO}_{2}$ layer.

Third, another photolithography process was conducted to form the metal cantilever structures of metadevice patterns. After this, both $\mathrm{Al}$ and $\mathrm{Al}_{2} \mathrm{O}_{3}$ layer are dry etched to form the designed metadevice pattern. Finally, the $\mathrm{SiO}_{2}$ sacrificial layer was etched away using vapor hydrofluoric acid (VHF), thereby suspending the cantilevers over the spacer with an air gap between them due to the stress difference. Since the bimorphs are in physical contact with spacer layer at the anchor region, the VHF release process is not time-controlled which 
ensures higher yield of the devices.

\subsection{Simulation tools}

Most results require to be repeated and verified in the numerical simulation tools, and the underlying mechanism is easily to be understood by analyzing the field distributions captured by the simulation tools. The commercially available software CST Microwave Studio was used to do the simulations by the frequency domain solver and time domain solver according to the requirements of analysis. In the frequency domain solver, unit cell boundary condition is applied for the model, and ports with 18 modes are set for the highest accuracy. Tetrahedral mesh should be chosen for frequency domain solver with the default mesh size, and higher mesh value will be required for extremely fine structure that will consume more memory as the price. The frequency domain solver provides a better resolution that can capture most of the fine features in the spectrum. In the time-domain solver, periodic boundary condition is applied for the unit cell model. Plane wave source and probes are needed to be set with the chosen polarization states according to the requirements. Hexahedral mesh should be chosen for time domain solver with the default mesh size, and also higher mesh size value is required for finer structures. The time domain solver better reproduces experimental progress in the time domain THz-TDs system and the scan length is also tunable for the requirement of different resolutions. 


\section{Chapter 2: Polarization optics}

Polarization together with intensity, coherence and frequency are fundamental properties of light. The remarkable properties of polarization have led to a deeper understanding of light and rendered a series of applications. Starting from the discovery of the double refraction in calcite crystal in $1669,{ }^{39}$ polarization of light obtained a long-term development by the pioneer works of Christian Huygens, Isaac Newton, Thomas Young, Etienne-louis Malus and Sir David Brewster. With the emergence of wave theory and wave equation, it is possible to describe the behaviors of light at the interface of two mediums, and from which we can understand the very interesting properties of light, polarization.

\subsection{Polarization ellipse}

Polarization, the vectorial nature of light, should be described by three independent oscillations along the Cartesian coordinate: $u_{x}(\overrightarrow{\mathrm{r}}, t), u_{y}(\overrightarrow{\mathrm{r}}, t)$ and $u_{z}(\overrightarrow{\mathrm{r}}, t)$, and thus the propagation of the oscillations requires three wave equations written as: ${ }^{40}$

$$
\nabla^{2} u_{i}(\overrightarrow{\mathrm{r}}, t)=\frac{1}{v^{2}} \frac{\partial^{2} u_{i}(\overrightarrow{\mathrm{r}}, t)}{\partial t^{2}} \quad i=x, y, z
$$

where $v$ is the velocity of light and $\vec{r}=\vec{r}(x, y, z)$. The components along $x$ and $y$ directions are regarded as the transverse components and that along $z$ direction is regarded as the longitudinal component when the light propagates in the $z$ direction. By solving Maxwell's equations, only the transverse components of light exist while propagating in free space and thus the optical field is written as: 
$u_{x}(z, t)=u_{0 x} \cos \left(\omega t-k z+\delta_{x}\right)$

$u_{y}(z, t)=u_{0 y} \cos \left(\omega t-k z+\delta_{y}\right)$

where $u_{0 x}$ and $u_{0 y}$ are the amplitude and $\delta_{x}$ and $\delta_{y}$ are the arbitrary phase items. To present the electromagnetic wave, we consider the electric component for simplicity and the transverse components are rewritten as:

$$
\begin{aligned}
& \frac{E_{x}}{E_{0 x}}=\cos (\omega t-k z) \cos \delta_{x}-\sin (\omega t-k z) \sin \delta_{x} \\
& \frac{E_{y}}{E_{0 y}}=\cos (\omega t-k z) \cos \delta_{y}-\sin (\omega t-k z) \sin \delta_{y} .
\end{aligned}
$$

We can then obtain the equations

$$
\begin{aligned}
& \frac{E_{x}}{E_{0 x}} \sin \delta_{y}-\frac{E_{y}}{E_{0 y}} \sin \delta_{x}=\cos (\omega t-k z) \sin \left(\delta_{y}-\delta_{x}\right) \\
& \frac{E_{x}}{E_{0 x}} \cos \delta_{y}-\frac{E_{y}}{E_{0 y}} \cos \delta_{x}=\sin (\omega t-k z) \sin \left(\delta_{y}-\delta_{x}\right)
\end{aligned}
$$

The addition of squared equations (2-6) and (2-7) forms

$$
\left(\frac{E_{x}}{E_{0 x}}\right)^{2}+\left(\frac{E_{y}}{E_{0 y}}\right)^{2}-2 \frac{E_{x}}{E_{0 x}} \frac{E_{y}}{E_{0 y}} \cos \delta=\sin ^{2} \delta
$$

where $\delta$ is defined as the phase difference, $\delta=\delta_{y}-\delta_{x}$. It is known that equation (2-8) describes an ellipse which means that the locus of points of the electric field is an ellipse as it propagates along $z$ direction. This is the so-called optical polarization, and the equation $(2-8)$ is the polarization ellipse. 


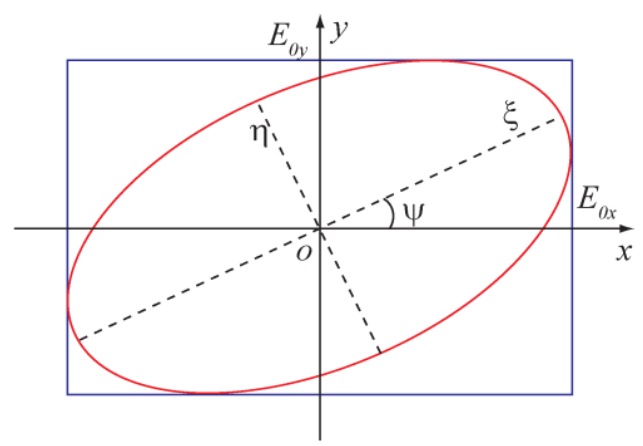

Figure 2-1. Polarization ellipse.

As shown in Figure 2-1 where the polarization ellipse inscribes within a rectangle with lengths $2 E_{0 x}$ and $2 E_{0 y}$, the ellipse is rotated by an angle $\psi$ with respect to the coordinate originating from the cross term $\left(E_{x} E_{y}\right)$ in equation (2-8). Based on the equation, we can deduce that $\tan 2 \psi=\frac{2 E_{0 x} E_{0 y} \cos \delta}{E_{0 x}^{2}-E_{0 y}^{2}}$ where $\psi$ is the angle of rotation. Another important parameter to describe the ellipse is named as the angle of ellipticity, $\chi$, that is defined as the ratio between the short axis and long axis $\left(\tan \chi=\frac{ \pm \eta}{\xi},-\frac{\pi}{4} \leq \chi \leq \frac{\pi}{4}\right)$ and calculated by $\sin 2 \chi=\frac{2 E_{0 x} E_{0 y} \sin \delta}{E_{0 x}^{2}+E_{0 y}^{2}}$. The polarization states are described either by the pellucid parameters, angle of orientation $\psi$ and ellipticity $\chi$, or $E_{0 x}, E_{0 y}$ and $\delta$ that are easy to be measured in an experimental THz-TDS system.

We here define the light as right-handed circularly polarized (RCP) if the end point of electric field forms the clockwise trajectory while looking in the direction where the light comes and vice versa. From the aspect of values, the RCP corresponds to the case of 
$0<\chi \leq \frac{\pi}{4}$ or

$\sin \delta>0$ and LCP (left-handed circular polarization) corresponds to the case of $-\frac{\pi}{4} \leq \chi<0$ or $\sin \delta<0$.

\subsection{Stokes parameters}

In nature, light is usually unpolarized or partially polarized such as sunlight and light from a bulb, and the polarization ellipse is no longer applicable to describe the behavior of such kind of light. Additionally, the electric vector of light traces out the polarization ellipse in a time interval of the order $10^{-15} \mathrm{sec}$ that prevents us to follow and observe the polarization evolution. An alternative method is therefore demanded to break the limitations by using average values of optical field in terms of observables that was discovered by Sir George Gabriel Stokes. ${ }^{41}$ Four measurable quantities known as the Stokes parameters are defined to depict any state of light including unpolarized and partially polarized light.

Considering the instantaneous amplitude of light, the polarization ellipse is written as:

$$
\left(\frac{E_{x}(t)}{E_{0 x}}\right)^{2}+\left(\frac{E_{y}(t)}{E_{0 y}}\right)^{2}-2 \frac{E_{x}(t)}{E_{0 x}} \frac{E_{y}(t)}{E_{0 y}} \cos \delta=\sin ^{2} \delta
$$

In order to present the equation from observables, we take the average value over time of the oscillation where $\left\langle E_{i}(t) E_{j}(t)\right\rangle=\lim _{T \rightarrow \infty} \frac{1}{T} \int_{0}^{T} E_{i}(t) E_{j}(t) d t, i, j=x, y \cdot{ }^{40}$ Finally, we get:

$$
\left(E_{0 x}^{2}+E_{0 y}^{2}\right)^{2}-\left(E_{0 x}^{2}-E_{0 y}^{2}\right)^{2}-\left(2 E_{0 x} E_{0 y} \cos \delta\right)^{2}=\left(2 E_{0 x} E_{0 y} \sin \delta\right)^{2}
$$

Through defining: 


$$
\begin{aligned}
& S_{0}=E_{0 x}^{2}+E_{0 y}^{2} \\
& S_{1}=E_{0 x}^{2}-E_{0 y}^{2} \\
& S_{2}=2 E_{0 x} E_{0 y} \cos \delta \\
& S_{3}=2 E_{0 x} E_{0 y} \sin \delta
\end{aligned}
$$

the equation is rewritten as $S_{0}{ }^{2}=S_{1}{ }^{2}+S_{2}{ }^{2}+S_{3}{ }^{2}$. The four parameters defined here is the so-called Stokes parameters described only by quantities with $S_{0}$ the intensity of light, $S_{1}$ the intensity of horizontal or vertical linear polarization, $S_{2}$ the intensity of $+45^{\circ}$ or $-45^{\circ}$ linear polarization and $S_{3}$ the intensity of right-handed or left-handed circular polarization (RCP or LCP). For the partially polarized light, the relations are rewritten as $S_{0}{ }^{2} \geq S_{1}^{2}+S_{2}^{2}+S_{3}^{2}$ and completely polarized light corresponds to the equality.

For the polarization ellipse, the two featured parameters can also be represented by Stokes parameters:

$$
\begin{aligned}
& \tan 2 \psi=\frac{2 E_{0 x} E_{0 y} \cos \delta}{E_{0 x}^{2}-E_{0 y}^{2}}=\frac{S_{2}}{S_{1}} \\
& \sin 2 \chi=\frac{2 E_{0 x} E_{0 y} \sin \delta}{E_{0 x}^{2}+E_{0 y}^{2}}=\frac{S_{3}}{S_{0}}
\end{aligned}
$$

With the Stokes parameters, we can also describe the degree of polarization $(P)$ for partially polarized or unpolarized light by

$$
P=\frac{I_{p o l}}{I_{t o t}}=\frac{\left(S_{1}^{2}+S_{2}^{2}+S_{3}^{2}\right)^{1 / 2}}{S_{0}} \quad 0 \leq P \leq 1
$$

where $I_{p o l}$ is intensity of the polarized components and $I_{t o t}$ is the total intensity of the light.

\subsection{Jones Matrix}

The propagation of the plane wave components along $z$-axis is usually presented as: 


$$
\begin{aligned}
& E_{x}(z, t)=E_{0 x} e^{i\left(\omega t-k z+\delta_{x}\right)} \\
& E_{y}(z, t)=E_{0 y} e^{i\left(\omega t-k z+\delta_{y}\right)}
\end{aligned}
$$

For simplicity, we rearrange equations $(2-15)$ in a column matrix:

$\vec{E}=\left(\begin{array}{l}E_{x} \\ E_{y}\end{array}\right)$

that is named as the Jones column matrix (Jones vector). ${ }^{42}$ In the matrix, the maximum amplitude $E_{0 x}$ and $E_{0 y}$ are real quantities and the exponent part indicates the phase item which causes the complex quantities of the plane wave. In order to clearly state the Jones matrix, we first find the normalization of it by the following steps. First, the total intensity of the plane wave is calculated by

$I=\left(\begin{array}{ll}E_{x}^{*} & E_{y}^{*}\end{array}\right)\left(\begin{array}{l}E_{x} \\ E_{y}\end{array}\right)=E_{x} E_{x}^{*}+E_{y} E_{y}^{*}$

where $\left(\begin{array}{ll}E_{x}^{*} & E_{y}^{*}\end{array}\right)$ is the complex transpose of the Jones matrix. Second, we can get $I=E_{0 x}^{2}+E_{0 y}^{2}=E_{0}^{2}$ and usually it is customary to define that $E_{0}^{2}=1$ in order to normalize the Jones matrix. From the Jones matrix elements, we can know that only completely polarized light can be presented. According to the Jones matrix, several specific polarization states (normalized) can be expressed in the Table 2-1 below:

Table 2-1. Specific polarization states presented by Jones vector

\begin{tabular}{lcccccc}
\hline \hline Polarization & LHP & LVP & LP $\left(+45^{\circ}\right)$ & LP $\left(-45^{\circ}\right)$ & RCP & LCP \\
\hline Jones vector & $\left(\begin{array}{l}1 \\
0\end{array}\right)$ & $\left(\begin{array}{l}0 \\
1\end{array}\right)$ & $\frac{1}{\sqrt{2}}\left(\begin{array}{l}1 \\
1\end{array}\right)$ & $\frac{1}{\sqrt{2}}\left(\begin{array}{c}1 \\
-1\end{array}\right)$ & $\frac{1}{\sqrt{2}}\left(\begin{array}{l}1 \\
i\end{array}\right)$ & $\frac{1}{\sqrt{2}}\left(\begin{array}{c}1 \\
-i\end{array}\right)$ \\
\hline \hline
\end{tabular}

The Jones vector has another important property that we can simply judge whether the 
components are orthogonal by doing the calculation $A^{*} B$ of the corresponding Jones vectors, and if $A^{*} B=0$, the two components are orthogonal with each other, for example, RCP and LCP $\frac{1}{\sqrt{2}}\left(\begin{array}{ll}1 & i\end{array}\right)^{*} \frac{1}{\sqrt{2}}\left(\begin{array}{c}1 \\ -i\end{array}\right)=0$. Regarding the coherent components, we can employ Jones vector to superpose the amplitudes, for example, the superposition of LHP and LVP,

$$
E=E_{H}+E_{V}=\left(\begin{array}{c}
E_{0 x} e^{i\left(\omega t-k z+\delta_{x}\right)} \\
0
\end{array}\right)+\left(\begin{array}{c}
0 \\
E_{0 y} e^{i\left(\omega t-k z+\delta_{y}\right)}
\end{array}\right)=\left(\begin{array}{c}
E_{0 x} e^{i\left(\omega t-k z+\delta_{x}\right)} \\
E_{0 y} e^{i\left(\omega t-k z+\delta_{y}\right)}
\end{array}\right)
$$

which describes the elliptically polarized light.

Jones matrix is usually used to describe the behavior of some polarization devices, such as polarizers, wave plate and rotators. Such devices response to incident light linearly without any nonlinear materials included and thus we can relate the emergent components to incident ones by:

$$
\begin{aligned}
& E_{x}^{T}=j_{x x} E_{x}^{i}+j_{x y} E_{y}^{i} \\
& E_{y}^{T}=j_{y x} E_{x}^{i}+j_{y y} E_{y}^{i}
\end{aligned}
$$

where $E_{x}^{T}$ and $E_{y}^{T}$ are the emergent components from the polarization devices and $E_{x}^{i}$ $E_{y}^{i}$ are the polarization components of incident light. The parameters $j_{i k}, i, k=x, y$ are the linear factors to link emergent and incident light. Transforming the equations (2-19) into matrix form, we can get:

$$
\left(\begin{array}{c}
E_{x}^{T} \\
E_{y}^{T}
\end{array}\right)=\left(\begin{array}{cc}
j_{x x} & j_{x y} \\
j_{y x} & j_{y y}
\end{array}\right)\left(\begin{array}{c}
E_{x}^{i} \\
E_{y}^{i}
\end{array}\right)
$$

where the $2 \times 2$ matrix $J=\left(\begin{array}{ll}j_{x x} & j_{x y} \\ j_{y x} & j_{y y}\end{array}\right)$ is named as the Jones matrix to describe the 
properties of the polarization devices.

For an ideal linear polarizer with rotated by an angle $\theta$ relative to horizontal plane, the Jones matrix is presented by $J_{P}(\theta)=\left(\begin{array}{cc}\cos ^{2} \theta & \sin \theta \cos \theta \\ \sin \theta \cos \theta & \sin ^{2} \theta\end{array}\right)$. For an ideal wave plate, it is related to the phase item of the light where the phase increases by $\varphi / 2$ along the fast axis of the crystal and retards by $-\varphi / 2$ along the slow axis. Therefore, the corresponding Jones matrix is written as $J_{W}(\varphi)=\left(\begin{array}{cc}e^{i \varphi / 2} & 0 \\ 0 & e^{-i \varphi / 2}\end{array}\right)$. When the total phase delay $\varphi$ is equal to $90^{\circ}$ or $180^{\circ}$, they correspond to a quarter wave plate and half wave plate, respectively. The other polarization device is a rotator who can rotate the incident polarization ellipse by an angle $\beta$ whose Jones matrix is written as $J_{R}=\left(\begin{array}{cc}\cos \beta & \sin \beta \\ -\sin \beta & \cos \beta\end{array}\right)$. The Jones matrix for the typical polarization devices are summarized in Table 2-2.

Table 2-2. Jones matrix for typical polarization devices.

\begin{tabular}{lccc}
\hline \hline Devices & Linear polarizer & Wave plate & Rotator \\
\hline Jones & $\left(\begin{array}{cc}\cos ^{2} \theta & \sin \theta \cos \theta \\
\sin \theta \cos \theta & \sin ^{2} \theta\end{array}\right)$ & $\left(\begin{array}{cc}e^{i \varphi / 2} & 0 \\
0 & e^{-i \varphi / 2}\end{array}\right)$ & $\left(\begin{array}{cc}\cos \beta & \sin \beta \\
-\sin \beta & \cos \beta\end{array}\right)$ \\
matrix &
\end{tabular}

The advances of Jones matrix reflect in the simplicity to present the polarization operation.

With the given Jones matrix expression $J$ for a polarizing device, the Jones matrix for the new configuration of the device is calculated through the simple operation:

$J_{\text {New }}=D^{-1} J D$ 
where matrix $D$ is the operator for the specific action. For example, the operator would be $D_{\theta}=\left(\begin{array}{cc}\cos \theta & \sin \theta \\ -\sin \theta & \cos \theta\end{array}\right)$ if we want to find the Jones matrix of the device that is rotated by an angle $\theta$ relative to $z$-axis (the wave propagation axis); and the operator would be $D_{M}=\left(\begin{array}{cc}1 & 0 \\ 0 & -1\end{array}\right)$ if we consider the Jones matrix of the mirror image of the primary device.

\subsection{Poincare sphere}

To map the polarization parameters to a sphere, it is necessary to do a coordinate transformation from Cartesian coordinate to Sphere coordinate. ${ }^{43,}{ }^{44}$ We first build a unit sphere with diameter equal to 1 that is tangential to the complex plane of the Cartesian coordinate at point $O$ as shown in Figure 2-2. $O O^{\prime}$ forms the diameter of the sphere and points $P_{1}$ and $P_{2}$ are projections of $p_{1}$ and $p_{2}$, respectively. According to the principles of stereographic projection, ${ }^{45}$ the arc $\overline{O M}$ with length $2 v$ and spherical angle of $\overline{M O O} \delta$ on the sphere is the projection of vector Om on the Cartesian coordinate where the point $O^{\prime}$ is the antipode of the sphere. We now take the center of the sphere as origin of the coordinate, so the coordinates of $m$ are transferred as:

$$
\begin{aligned}
& x=-\frac{1}{2} \\
& y=\tan v \cos \delta \\
& z=\tan v \sin \delta
\end{aligned}
$$




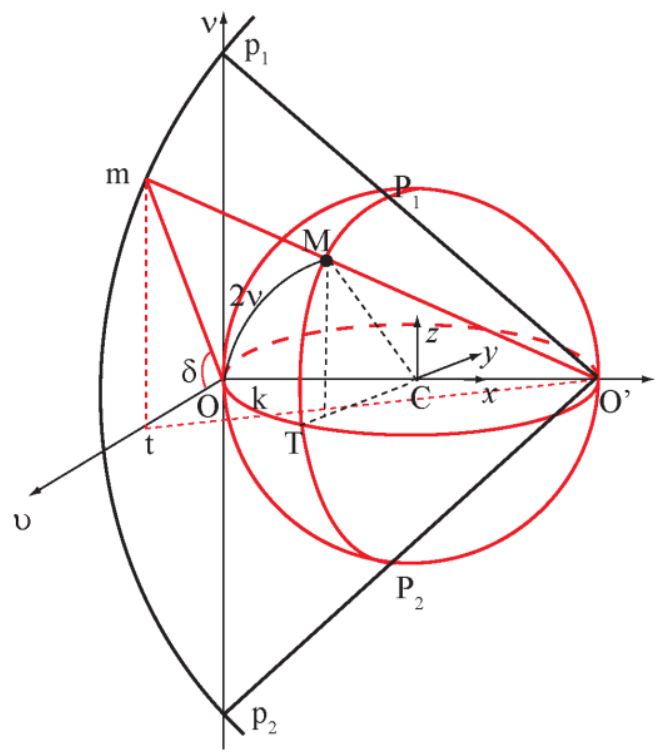

Figure 2-2. Schematic diagram of stereographic projection for the polarization ellipse represented on a complex plane to a sphere surface. Any elliptically polarized light is presented at point $m$ on the complex plane with vector $\mathbf{O m}$ and angle $\delta$. The corresponding data are projected on the sphere as point $\mathrm{M}$ with arc $\overline{\mathrm{OM}}$ of length $2 v$ and angle of $\overline{\mathrm{MOO}^{\prime}}$. From Figure 2-2, we can see that point $M$ is in fact the projection through the straight line $m M O^{\prime}$ and the sphere is written as $x^{2}+y^{2}+z^{2}=\left(\frac{1}{2}\right)^{2}$. The point $M$ in the $x y z$ coordinate is finally calculated as (calculation details can be found at Chapter 12.3 in Ref. 1):

$$
\begin{aligned}
& x=\frac{1}{2} \cos 2 v \\
& y=\frac{1}{2} \sin 2 v \cos \delta \\
& z=\frac{1}{2} \sin 2 v \sin \delta
\end{aligned}
$$

Recalling equation (2-11) where we give the expressions of Stokes parameters, we set $S_{0}=A^{2}+B^{2}=C^{2}$ where $A=E_{0 x}, \quad B=E_{0 y}, \quad \tan v=\frac{E_{0 y}}{E_{0 x}}$ and $C$ represents the length of the 
hypotenuse of a right triangle with $A$ and $B$ the right angle sides. We can rewrite the Stokes parameters as:

$$
\begin{aligned}
& S_{0}=C^{2} \\
& S_{1}=C^{2} \cos 2 v \\
& S_{2}=C^{2} \sin 2 v \cos \delta \\
& S_{3}=C^{2} \sin 2 v \sin \delta
\end{aligned}
$$

where $\delta=\delta_{y}-\delta_{x}$. By setting $C^{2}=\frac{1}{2}$, we easily find that equations for Stokes parameters are identical with those for $x, y$ and $z$, and thus the point $M$ on the sphere can represent the value of Stokes parameters of light where $S_{0}$ is the radius of the sphere.

Usually, the intensity of light is normalized and thus the radius of Poincare sphere is 1 which render the Stokes parameters written as:

$$
\begin{aligned}
& S_{0}=1 \\
& S_{1}=\cos 2 \varepsilon \cos 2 \theta \\
& S_{2}=\cos 2 \varepsilon \sin 2 \theta \\
& S_{3}=\sin 2 \varepsilon
\end{aligned}
$$

where $\varepsilon$ and $\theta$ are the ellipticity and azimuth of the polarization ellipse, respectively.

\subsection{Summary}

In this chapter, we introduced the fundamental knowledge of polarization optics. We first deduce the polarization ellipse equation from the wave equations. From the polarization ellipse equation, we can then describe the polarization states from the shape that it presents in the Cartesian coordinate. In order to numerically describe the polarization states from the parameters that we can directly experimentally measure, we also introduce the Stokes parameters that can not only describe polarized light but also describe the polarization 
degree of unpolarized or partially polarized light. The polarization transforming due to the presence of a medium is described by the Jones matrix that connects the incidence and output light. For visualized presentation of polarization states, the Poincare sphere is also introduced which provides a powerful tool for the expression of polarization states. 


\section{Chapter 3: Symmetry based polarization control}

Symmetry can be found in almost all the disciplines in nature, which governs the laws to study and explore the universe. The beauty of symmetry reveals not only on the macroscopic architectures, arts and appearance of biology but also on the microcosmic organism, molecule and atoms. Symmetry is accurately defined through mathematical method with a property that is invariant to a transformation with respect to the passage of time. ${ }^{46,47}$ The study of symmetry is especially significant in chemistry, biology and physics disciplines since plentiful of interesting phenomena can be explored and explained from the aspect of symmetry, and the slight change of symmetry in molecular or crystal structure would induce enormous changes in their intrinsic properties. ${ }^{31,} 48$ In addition to the symmetry studies in spatial configuration, the symmetry in physical properties also matters much in determining the properties such as the loss of materials. Recently, there is intense discussion of parity-time $(P T)$ symmetry breaking in non-Hermitian wave equation which was achieved by breaking the symmetry of loss and gain in the materials, and it is able to find applications in microring lasing. ${ }^{49-52}$ In the normal crystal lattice, the atoms are locked to form the intrinsic properties of the materials, and the polarization $\vec{p}$ is determined by $\vec{p}=\varepsilon_{0} \chi \vec{E}$ where $\vec{E}$ is external electric field, $\varepsilon_{0}$ is the permittivity of vacuum and $\chi$ is the susceptibility tensor of the crystal. ${ }^{40}$ Varied crystalline structure will result in different susceptibility tensors that determine the isotropic or anisotropic features of the media, and thus arbitrary control of the lattice symmetry helps to tailor the material properties at will. However, it is not straightforward to control the configuration of crystal structures of 
natural materials. In order to obtain the customized material properties, metamaterial is the excellent choice where lots of extraordinary phenomena that natural materials are not able to achieve have been realized such as negative refractive index, ${ }^{53}$ perfect lenses, ${ }^{54}$ flat lenses ${ }^{55,56}$ and extraordinary propagation..$^{23,57}$

\subsection{Background}

With the help of metamaterials, we can study and verify the relationship between symmetry and the polarization of light easily. For simplicity, we degraded the study from threedimension (3D) metamaterial to two-dimension (2D) metasurface which also reduces the complexity of fabrication. The $2 \mathrm{D}$ metasurface simplifies the tensor $\chi$ from a $3 \times 3$ matrix to a $2 \times 2$ matrix. The polarization properties of metamaterials have been intensely studied in the recent years, such as chirality, ${ }^{33,} 58$ enhanced circular dichroism, ${ }^{59}$ asymmetric transmission ${ }^{36,60,61}$ and enhanced Faraday effect. ${ }^{29}$ Usually, the fourfold rotational symmetric unit cell in the metasurface array is adopted to discuss the planar chirality. ${ }^{62-64}$ For asymmetric transmission, circularly polarized light has been studied in details in planar metasurfaces $^{60,} 61$ and linearly polarized light was also discussed in multilayer metamaterials by breaking the propagation symmetry. ${ }^{36}$ Another method to excite the optical activity of planar metasurface is though tilting the incident light with an angle relative to the normal of metasurface so that the metasurface is excited with both electric and magnetic dipoles interacting with each other. ${ }^{65}$ The underlying physics has been interpreted thoroughly, however, the effect of optical activity in these designs is extremely weak that is not efficient for real applications in polarization control devices. Enhanced 
optical activity or circular dichroism is obtained through the multilayer or 3D metamaterials that improves the light-matter interaction and excites the magnetic mode. ${ }^{59,66}$ These designs possess enhanced polarization attenuation effects, however, the fabrication complexity and difficulty have limited their applications. Therefore, a simpler and more efficient metasurface is required to obtain the giant optical activity and asymmetric transmission that can be fabricated through the conventional and productive techniques.

\subsection{The theory and design}

Unlike the previous discussion, we here proposed a planar metasurface that is easily fabricated by conventional photolithography with unit cell holding the lowest order rotational symmetry, i.e., twofold symmetry $(C 2)$. Their optical activity and asymmetric transmission are discussed in the terahertz regime. With the strong excitation of crosspolarized component by linearly polarized incidence, such a metasurface is demonstrated with a giant optical activity, polarization modulation effect and asymmetric transmission. The cross-polarized component is a straightforward way to determine the optical activity, and it is also a key element for applications in flat lenses and hologram by metasurfaces in transmission mode. Compared to the fourfold symmetric unit cell, the twofold design provides an extra degree to tailor the optical axes of the unit cell that could be employed to modulate the amplitude of the cross-polarized component. Through optimizing the parameters, effects of the intrinsic optical activity, polarization modulation effect and asymmetric transmission are readily to be modulated. 


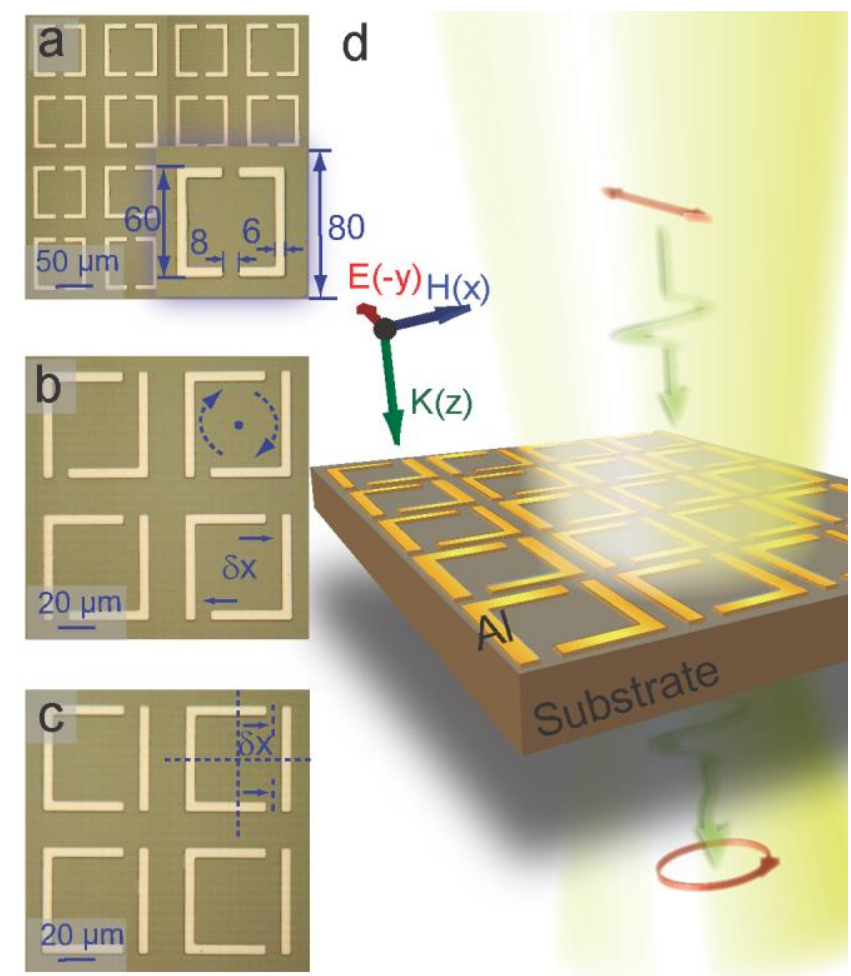

Figure 3-1. Images of samples by breaking a certain symmetry through varying the gap positions of a double-gap split ring resonator. (a) Detailed geometrical parameters of the original DSRR; (b) microscopic image of DSRR with mirror symmetry broken and (c) image of DSRR with rotational symmetry broken. (d) Schematic diagram of transmission experiments. ${ }^{67}$

The metasurface array design is proposed on the basis of the double-gap split ring resonator (DSRR) unit cell which has two gaps located at the center of two arms as shown in Figure 3-1(a). Such a DSRR unit cell holds both mirror symmetry and C2 rotational symmetry named as $s$-SRR. Here, we focus on the relationship between geometrical symmetry and optical activity based on the proposed DSRR array. The strategy to realize the symmetry breaking is through tailoring the displacement of the split gaps of the DSRR unit cell. As shown in Figure 3-1(b), we break the intrinsic mirror symmetry of the unit cell by 
displacing both the two gaps in the opposite directions with the same distance $\delta x$ whose $C 2$ rotational symmetry remains, and thus we name it as $r$-SRR. Similarly, the $C 2$ rotational symmetry is broken by displacing the two gaps in the same direction with the same distance, and mirror symmetric axis along $x$-direction remains named as $x$-SRR as shown in Figure 3-1(c). With the three sets of metasurface array with different intrinsic symmetries in the unit cell, we then address the different transmission behaviors related to optical activity. An 8- $f$ antenna based terahertz time-domain spectroscopy system was used to obtain all the required transmission signals with the inserted wire-grid polarizers (details can be found in

\section{Chapter 1.3).}

As we have discussed in Chapter 2.3, a generalized Jones matrix $(J)$ can be applied to the metasurface that connects the incident and transmitted light with coherent light as source while assuming that all the materials are linear and reciprocal. The Jones matrix is written as $\quad J^{f}=\left(\begin{array}{ll}j_{x x} & j_{x y} \\ j_{y x} & j_{y y}\end{array}\right)$ where the superscript $f$ means the forward propagation. Considering the symmetry of the $r$-SRR ( $x$-SRR) where the $180^{\circ}$ rotation along $z$-axis (mirror image along $x$-axis) of the unit cell is identical to the original one, we can discuss the properties of the metasurface theoretically by Jones matrix. Recalling the operation of Jones matrix, the operator is $D_{\theta}=\left(\begin{array}{cc}\cos \theta & \sin \theta \\ -\sin \theta & \cos \theta\end{array}\right)$ to obtain the new Jones matrix while rotating the unit cell by an angle $\theta$ and the new Jones matrix is calculated by $J_{\text {New }}=D^{-1} J D$. For $r$-SRR, we use the operator $D_{180^{\circ}}=\left(\begin{array}{cc}-1 & 0 \\ 0 & -1\end{array}\right)$ to get the matrix of $180^{\circ}$ rotated counterpart, and then get the new 
Jones matrix as $J_{N e w}^{f}=\left(\begin{array}{cc}j_{x x} & j_{x y} \\ j_{y x} & j_{y y}\end{array}\right) \equiv J^{f}$ that is identical to the original one indicating no specific features appeared from the aspect of Jones matrix for a unit cell with $C 2$ rotational symmetry. Similarly, the operator is written as $D_{M}=\left(\begin{array}{cc}1 & 0 \\ 0 & -1\end{array}\right)$ to obtain the Jones matrix of the mirror image of original unit cell, and thus the Jones matrix is calculated as $J_{N e w}^{f}=\left(\begin{array}{cc}j_{x x} & -j_{x y} \\ -j_{y x} & j_{y y}\end{array}\right)$, which should be identical to the original Jones matrix for $x$-SRR and require the equation $J_{\text {New }}^{f}=\left(\begin{array}{cc}j_{x x} & -j_{x y} \\ -j_{y x} & j_{y y}\end{array}\right)=J^{f}=\left(\begin{array}{cc}j_{x x} & j_{x y} \\ j_{y x} & j_{y y}\end{array}\right)$. Therefore, a featured Jones matrix to describe the transmission behavior of $x$-SRR is obtained as $J^{f}=\left(\begin{array}{cc}j_{x x} & 0 \\ 0 & j_{y y}\end{array}\right)$ where crosspolarized transmission items appear to be zero. The cross-polarized components are important to obtain optical activity which describe the different properties between $r$-SRR and $x$-SRR.

\subsection{Experimental verification}

The metasurface samples were fabricated using the conventional photolithography method (details can be found in Chapter 1.5) whose images are displayed in Figure 3-1 with the schematic measurement setup. In order to verify the theoretical prediction from experiment, three sets of representative samples were fabricated ( $s$-SRR, $r$-SRR and $x$-SRR) with $\delta x=0$ (s-SRR), $\delta x=10 \mu \mathrm{m}$ and $\delta x=20 \mu \mathrm{m}(r$-SRR and $x$-SRR). Except the degree of symmetry (represented by $\delta x$ ), all the remaining parameters are the same for all the samples. As we have discussed theoretically above, the cross-polarized components reveal the contrast behaviors between the two featured symmetries, and thus we first probe the cross-polarized 
transmission signals through experiments and simulations where the finite-element frequency-domain (FEFD) solver was employed (details can be found in Chapter 1.6)

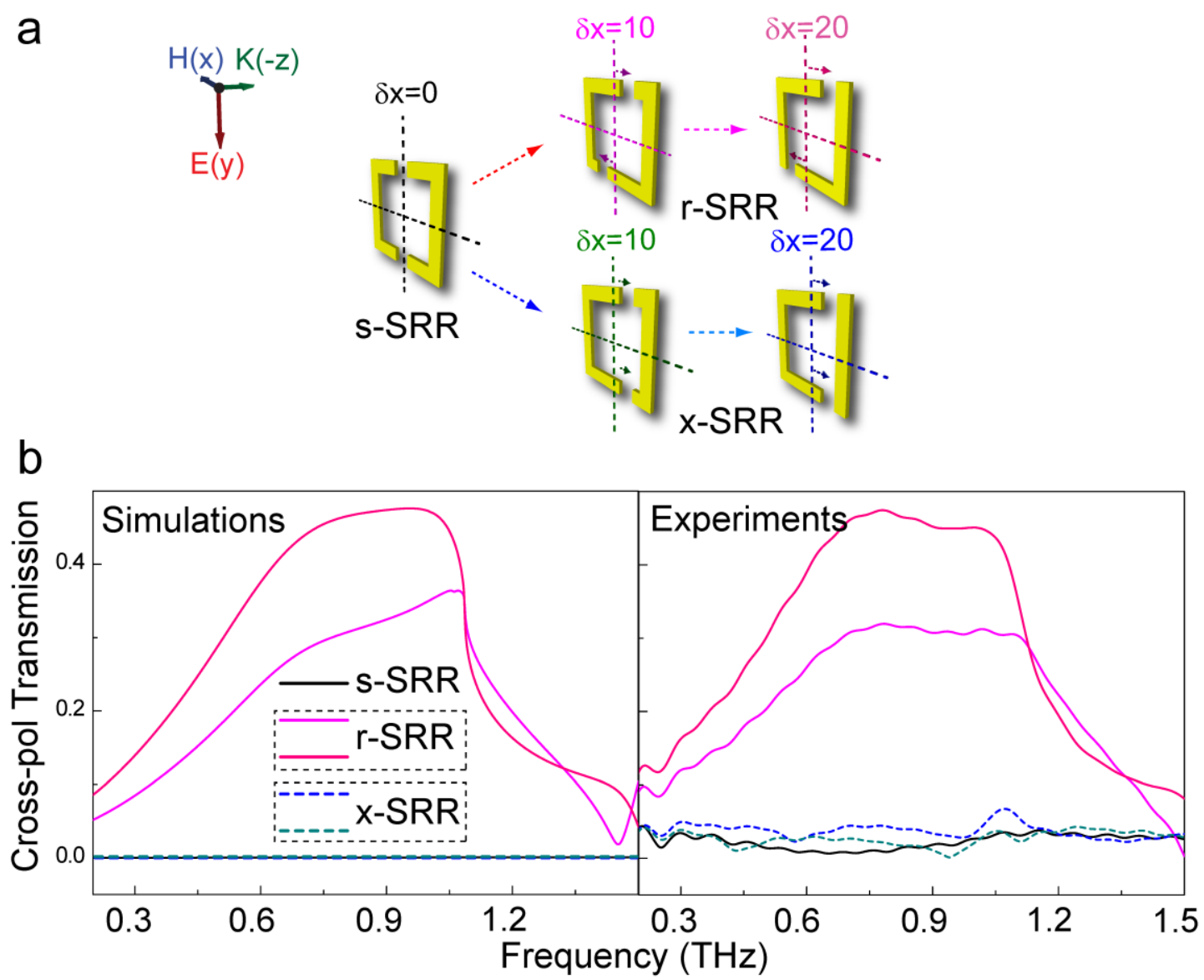

Figure 3-2. Experimental and simulated results of cross-polarized transmission signals at different symmetry configurations. (a) Schematic diagram of the symmetry evolution by displacing the split gaps in DSRR; (b) cross-polarized transmission signals by simulations and experiments, respectively. ${ }^{67}$

Simulations were first performed to give tentative results as shown in Figure 3-2(b), where we clearly observe the emergence of cross-polarized signals for $r$-SRR and no signal for $s$ SRR and $x$-SRR. The corresponding experimental results are also presented in Figure 32(b) that match well to the simulation results. In experiments, the incidence was set to be 
linearly polarized along $y$-axis, and transmitted signals polarized along $x$-axis were measured and normalized to the reference signals (co-polarized signal of bare substrate silicon). We then obtained one element of Jones matrix $j_{x y}$ in this measurement, and the amplitude coefficient was extracted from the absolute value of the element, i.e. $\left|j_{x y}\right|$. We note that here we use linear polarization as the base to describe the transmission signals by using the combination of three polarizers to measure the orthogonal polarization components. We also verify the distinct transmission behaviors by comparing the contrasting amplitude of $x$-SRR and $r$-SRR from the experimental results. Here, extremely weak cross-polarized signals were measured for $x$-SRR samples that mainly come from the system noise and non-ideal polarizers. For the $r$-SRR, the highest value of cross-polarized transmission amplitude reaches to 0.47 at the case of $\delta x=20 \mu \mathrm{m}$ that is close to the theoretical limit 0.5 for the planar metasurface where power conservation and reciprocity apply (details can be found in supplementary materials of Ref. 69) ${ }^{68,69}$ The cross-polarized transmission spectra with $x$-polarized incidence $\left(\left|j_{y x}\right|\right)$ are also measured that reveal the similar behaviors (as shown below in Figure 3-6). Thus far, we have verified the predictions from Jones matrix from both simulations and experiments.

In addition to the contrasting amplitude of cross-polarized components originating from the intrinsic symmetry of unit cell, another interesting phenomenon is observed from the measured data for $r$-SRR, where the overall amplitude of cross-polarized light is modulated by varying the gap displacement $(\delta x)$. As shown in Figure 3-2(b), the amplitude is modulated from 0.31 to 0.47 while displacing $\delta x$ from $10 \mu \mathrm{m}$ to $20 \mu \mathrm{m}$ which provides an 
alternative to modulate the resultant optical activity and output polarization states which will be discussed in the following subsections.

\subsection{Explanation from electric field distributions}

Cross-polarized components are the key ingredients not only in polarization optics and optical activity but also in the recent developed phase gradient supercell in transmission mode for applications in flat lenses and holograms. Therefore, it is necessary to clearly interpret the underlying mechanism so that we can optimize the performance in these applications. In simulations, we extract the electric field distributions of polarization along $x$ and $y$ directions, respectively, at the dipole resonance frequency $0.7 \mathrm{THz}$ excited by $y$ polarized incidence. The surface currents flow on the metal branches reveal a dipole mode at different configurations. For electric field distributions, as shown in Figure 3-3(a) for the near-field $y$-polarized component distribution, the fields mainly concentrate on the top and bottom arms of DSRR, and the strongest dipolar resonance occurs at $s$-SRR case while the resonance strength weakens obviously with the increasing of $\delta x$. This indicates the lowered scattering power along $y$-polarized component at larger $\delta x$. As for the case of field distributions along $x$-polarized direction in Figure 3-3(b), the fields distributed symmetrically for $s$-SRR that cancel with no net fields radiating to the far field, however, radiation occurs once the mirror symmetry is broken for $r$-SRR. Based on the understanding of electric field distribution, the cross-polarization evolution can be interpreted visually from the energy density distributions in Figure 3-3(c) where the fractions of energy reside only on top and bottom arms (excitation of $y$-polarized radiation, co-polarized component) 
for $s$-SRR, and the fractions shift to left and right arms gradually (excitation of $x$-polarized radiation, cross-polarized component) while displacing the gap positions.
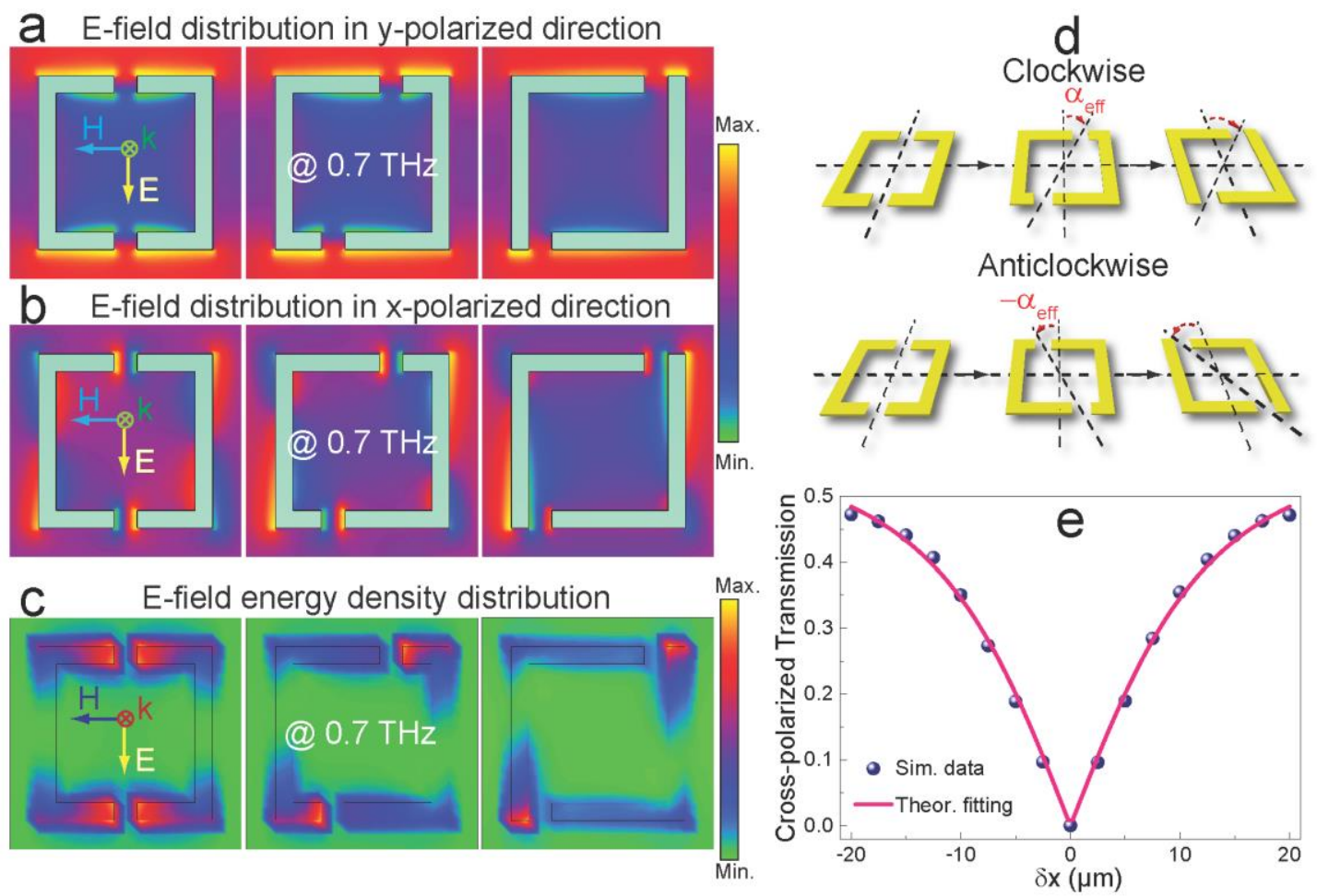

Figure 3-3. Underlying mechanism of cross-polarized component excitation. (a) and (b) electric field (E-field) distributions polarized along $y$-axis and $x$-axis, respectively. (c) Corresponding energy density distributions of $s$-SRR and $r$-SRRs. (d) Schematic definition of effective rotation degree and (e) extracted cross-polarized amplitude with the fitting curve using the proposed model.

According to the understanding of field distributions, we now move to the numerical interpretation between cross-polarized amplitude and gap displacement. Since the gap is displaced with $C 2$ rotational symmetry reserved, we define the effective rotation degree 
$\left(\alpha_{e f f}\right)$ as illustrated in Figure 3-3(d), and the degree is calculated through $\sin \alpha_{e f f}=\frac{\delta x}{\sqrt{\delta x^{2}+l_{e f f}^{2}}}$ where $l_{\text {eff }}$ is the effective length of the $r$-SRR. We note that here the effective rotation angle is only valid to the $y$-polarized incidence, and it will be different at different incident polarization angles due to the intrinsic anisotropy of the resonator. As we have discussed, cross-polarized amplitude increases when $\delta x$ is larger, and thus we give an initial assumption that the amplitude is proportional to sinusoidal function of the effective rotation degree, i.e. $\left|T_{x y}\right| \propto \sin \alpha_{e f f}$. In order to verify this assumption, we exact and plot the amplitude data of cross-polarized component at $0.7 \mathrm{THz}$ by displacing the split gaps from $\delta x=-20 \mu \mathrm{m}$ (anticlockwise $C 2$ symmetry) to $\delta x=20 \mu \mathrm{m}$ (clockwise $C 2$ symmetry) and fitting the data with the proposed model as shown in Figure 3-3(e). Using the fitting equation $\left|T_{x y}\right|=\left|k \frac{\delta x}{\sqrt{\delta x^{2}+l_{e f f}^{2}}}\right|,(-20 \leq \delta x \leq 20)$, it perfectly matches with the extracted data by giving $l_{\text {eff }}$ $=13.776 \mu \mathrm{m}$ and $k=0.588$ where $k$ is the proportionality coefficient. From this equation, we numerically obtain the dependence of cross-polarized amplitude on the displacement of gaps. Although rotational symmetry is not the precondition to induce cross-polarized component (the asymmetric DSRR can also induce it), the larger value of cross-polarized amplitude is able to be achieved for $r$-SRR with $C 2$ rotational symmetric at larger rotation degree. $^{67}$

\subsection{Asymmetric transmission and optical activity}

For a four-port system without external magnetic field applied, the Jones matrix is written as $J^{b}=\left(\begin{array}{cc}j_{x x} & -j_{y x} \\ -j_{x y} & j_{y y}\end{array}\right)$ when the light propagates from back to forward through the planar 
metasurface according to the reciprocity theorem. ${ }^{57,70}$ In fact, the unit cell observed from back of the sample is the mirror image of the unit cell observed from the front side, and thus the Jones matrix is also written as $J^{b}=\left(\begin{array}{cc}j_{x x} & -j_{x y} \\ -j_{y x} & j_{y y}\end{array}\right)$ by applying the matrix operator for a mirror image. Comparing the two matrices, we find $j_{x y}=j_{y x}$ which indicates that the cross-polarized transmission behavior will always be identical with different incident polarizations. With this feature, we then deduce that no asymmetric transmission of linearly polarized light exists that is defined by $\Delta=\left|J^{f}\right|^{2}-\left|J^{b}\right|^{2}$ due to $\Delta_{l i n}^{x}=-\Delta_{l i n}^{y}=\left|j_{y x}\right|^{2}-\left|j_{x y}\right|^{2} \equiv 0$. However, the Jones matrix is rewritten as $J_{\text {Circ }}^{f}=\left(\begin{array}{ll}j_{R R} & j_{R L} \\ j_{L R} & j_{L L}\end{array}\right)=\frac{1}{2}\left(\begin{array}{ll}\left(j_{x x}+j_{y y}\right)+i\left(j_{x y}-j_{y x}\right) & \left(j_{x x}-j_{y y}\right)-i\left(j_{x y}+j_{y x}\right) \\ \left(j_{x x}-j_{y y}\right)+i\left(j_{x y}+j_{y x}\right) & \left(j_{x x}+j_{y y}\right)-i\left(j_{x y}-j_{y x}\right)\end{array}\right)$

for circularly polarized light based on the linear polarization elements where $R$ represents the right-handed circularly polarized light (RCP) and $L$ represents the left-handed circularly polarized light (LCP). The asymmetric transmission for circularly polarized light is calculated by $\Delta_{\text {Circ }}^{R}=-\Delta_{\text {Circ }}^{L}=\left|j_{L R}\right|^{2}-\left|j_{R L}\right|^{2} \neq 0 .{ }^{60}$

In addition to the asymmetric transmission, the cross-polarized component will also introduce the planar chirality for the $r$-SRR although the intrinsic chirality requires the induced magnetic moment (electric dipole) parallel to the external electric (magnetic) fields where circularly polarized light corresponds to the eigen state. ${ }^{58,71,72}$ As for the planar metasurface, the intrinsic chirality is almost impossible to achieve with the circularly polarized eigen states, however, we can approach strong planar chirality by carefully designing the symmetry and parameters of metasurface. 

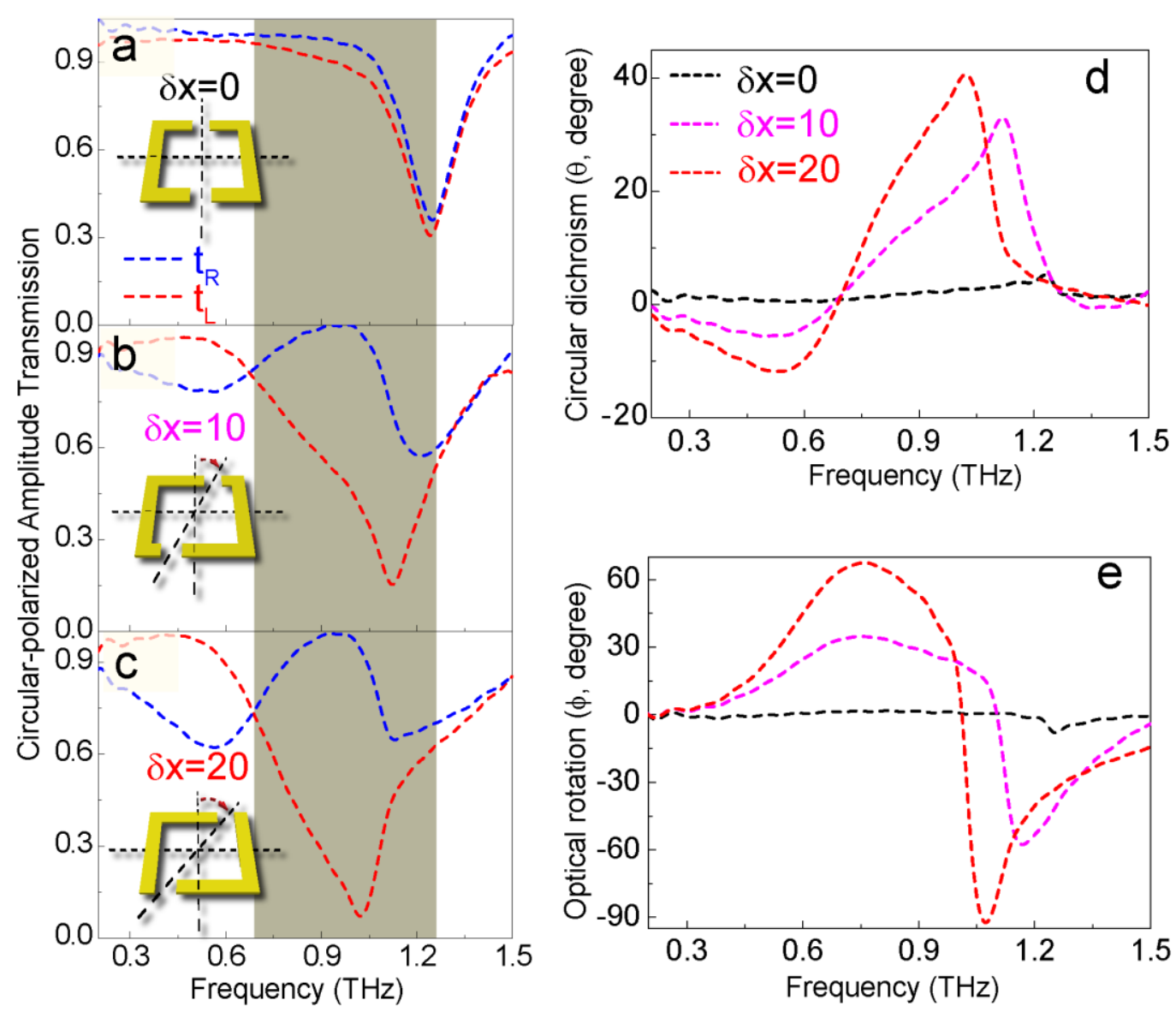

Figure 3-4. Study of circularly polarized light transmission behaviors for $\boldsymbol{r}$-SRR. (a),

(b) and (c) output spectra of RCP and LCP light with $\delta x=0 \mu \mathrm{m}, \delta x=10 \mu \mathrm{m}$, and $\delta x=20 \mu \mathrm{m}$, respectively; (d) and (e) corresponding circular dichroism and optical rotation spectra. ${ }^{67}$

We take two parameters: circular dichroism (CD) and optical rotation, to measure the planar chirality where $\mathrm{CD}$ is calculated by $\theta=\tan ^{-1}\left[\left(j_{R}-j_{L}\right) /\left(j_{R}+j_{L}\right)\right]$ indicating the difference between RCP and LCP of output light, and optical rotation is calculated by $\phi=\arg \left(j_{R}\right)-\arg \left(j_{L}\right)$ showing the rotation of major axis of polarization ellipse where $j_{R(L)}$ is the total transmission signals for RCP(LCP) light. As we discussed for circularly polarized light, the element $j_{R R}$ is always equal to $j_{L L}$ due to $j_{x y}=j_{y x}$ for the planar metasurface and thus, we deduce that the planar CD merely comes from the difference 
between $j_{R L}$ and $j_{L R}$. As shown in Figure 3-4(a)-(c), the circularly polarized transmission spectra for $r$-SRR with different gap displacements are shown, where the difference between the output RCP and LCP amplitude is clearly observed. In the shaded region, no obvious difference between RCP and LCP is measured at the case of $\delta x=0 \mu \mathrm{m}$. The difference becomes obvious once the mirror symmetry is broken in $s$-SRR, and reaches to the largest at the case of $\delta x=20 \mu \mathrm{m}$. From Figure 3-4(d), CD spectra are summarized where the difference between RCP and LCP is explicitly exhibited, and the largest value of CD reaches to $40.7^{\circ}$ at $0.9 \mathrm{THz}$ that raises substantially compared to the previously reported values. ${ }^{58}$ The other parameter to measure the planar chirality is the optical rotation, also known as optical activity. As shown in Figure 3-4(e), similar behavior is also observed in this spectrum with the largest rotation angle reaching to $53^{\circ}$. On the basis of the discussion above, the large CD value originating from the difference between $j_{L R}$ and $j_{R L}$ will also lead to the asymmetric transmission of circularly polarized light. With the incident light propagating forward and backward through the metasurface, the optical activity will also be inversed according to the definition.

\subsection{Application as a quarter wave plate}

With the large circular dichroism and optical rotation, the proposed $r$-SRR can be optimized to operate as a flat polarization convertor. In order to discuss the polarization states of output light, it is required to obtain both the orthogonal components in the transmission port $\left(j_{x y}\right.$ and $\left.j_{y y}\right)$ with a fixed incident polarization state. In the linearly polarized space, the cross-polarized component $\left(j_{x y}\right)$ have been shown in Figure 3-2(b). We present the co- 
polarized transmission component $\left(j_{y y}\right)$ in Figure 3-5(a) and (c). With the two orthogonal components, it is possible to calculate the polarization states of output light described by ellipticity using Stokes parameters ${ }^{39}$ as shown in Figure 3-5(b). The corresponding spectra obtained from simulations are also shown in Figure 3-5(c) and (d) for comparison that reveal the excellent match with the measured data.
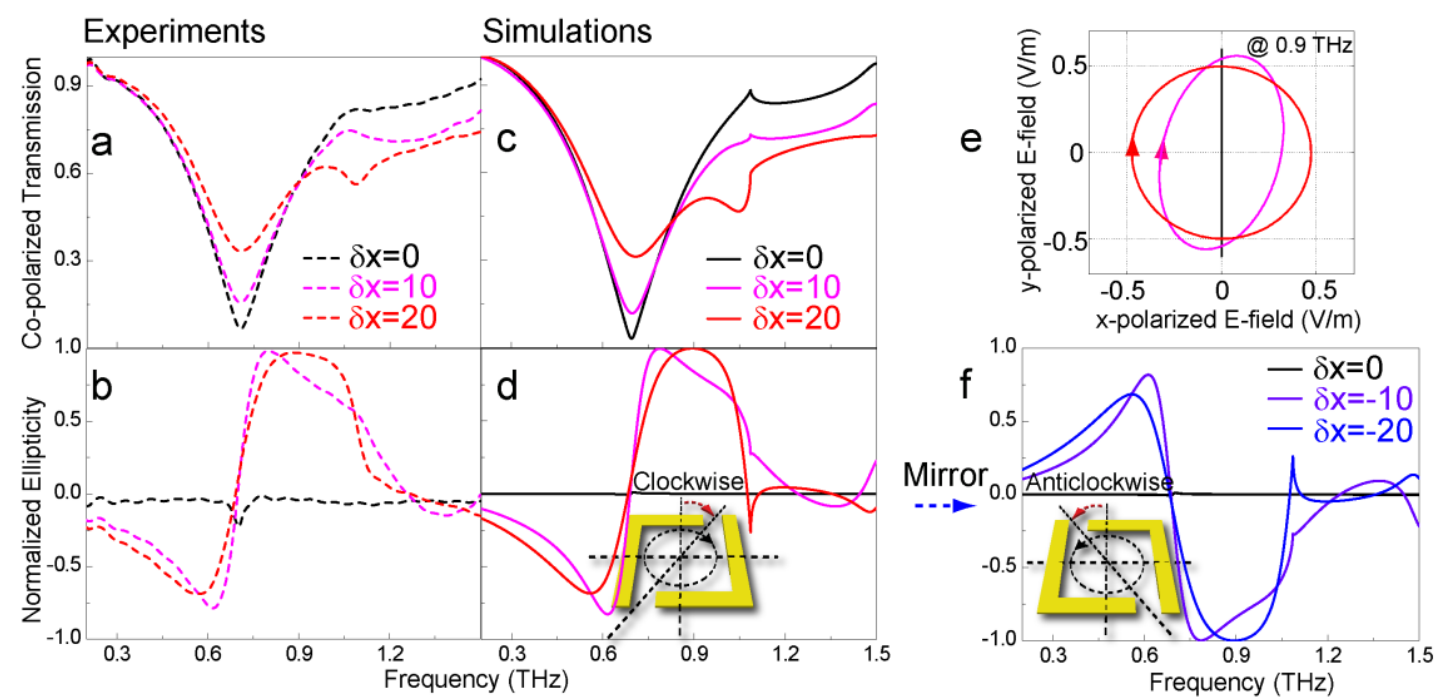

Figure 3-5. Performance of $\boldsymbol{r}$-SRR to function as a quarter wave plate. (a) and (b) measured co-polarized transmission amplitude and normalized ellipticities. (c) and (d) corresponding spectra based on simulations. (e) Calculated output polarization states and (f) ellipticities of output light from $r$-SRR while shined from backward with the same incident polarization.

From the ellipticity spectra, it is not difficult to find that no polarization conversion occurs for $s$-SRR, and the output light becomes right-handed elliptically and circularly polarized state at $0.9 \mathrm{THz}$ for $r$-SRR with $\delta x=10 \mu \mathrm{m}$ and $\delta x=20 \mu \mathrm{m}$, respectively. The visualized evolution of polarization state is presented in Figure 4-5(e). In addition to the polarization 
modulation in $r$-SRR, we can also observe that the operation bandwidth is broadened in the ellipticity spectra that provides a functionality of a quarter wave plate from $0.85 \mathrm{THz}$ to $0.95 \mathrm{THz}$ at the case of $\delta x=20 \mu \mathrm{m}$. Such a broadband behavior is interpreted from the optical axis rotation of the $r$-SRR that results in the excitation of a dipole mode along $x$ axis with $y$-polarized incidence. With the anisotropy of the unit cell as shown in Figure 36 , the output polarization states will be different by rotating the sample/incident polarization angle.

(a) $T_{x x}$

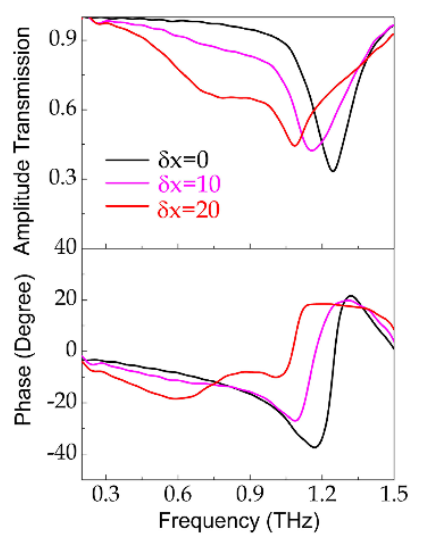

(c) $T_{y x}$

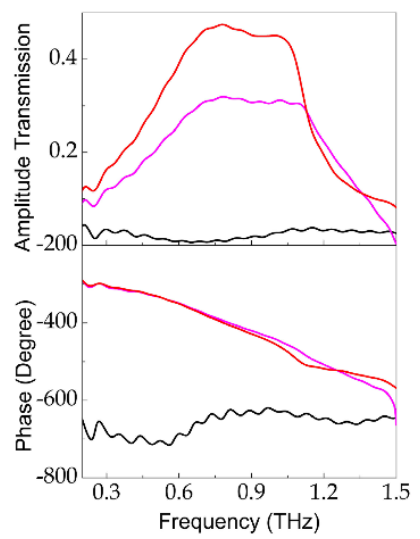

(b) $T_{x y}$

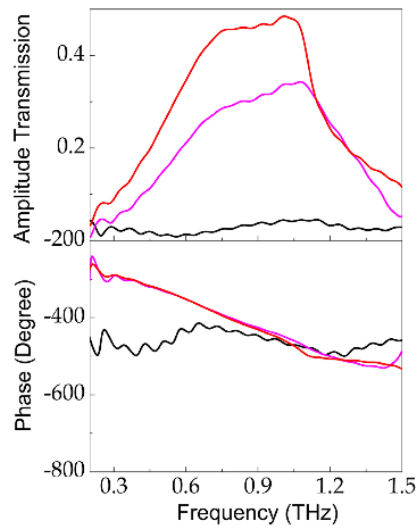

(d) $T_{y y}$

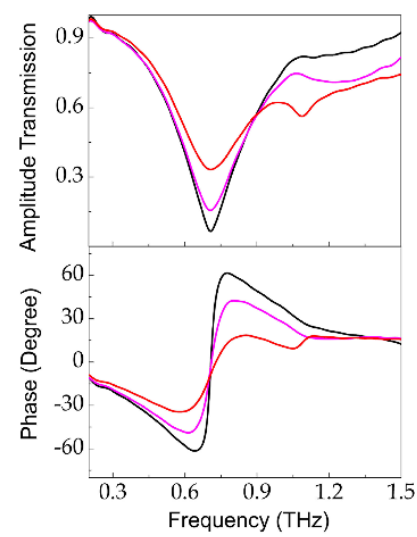

Figure 3-6. Measured transmission elements of Jones matrix.

The asymmetric transmission of circularly polarized light also leads to the inversion of ellipticities when the metasurface is excited from the opposite side with the same polarized 
incidence as shown in Figure 3-5(f). At this case, the output light is converted to LCP with $\delta x=20 \mu \mathrm{m}$. Although the operation bandwidth is still limited in the planar metasurface configuration, it reveals promising application values as a flat and ultrathin optical component. The broader operation band is achievable with multilayer metamaterials that will be discussed in Chapter 4 in this thesis. ${ }^{73-75}$

\subsection{Conclusions}

In this chapter, we have theoretically and experimentally discussed the cross-polarized components in the Jones matrix from the aspect of intrinsic symmetry in the unit cell of the metasurface array. Cross-polarized light cannot be induced for unit cell with mirror symmetry, and giant cross-polarized amplitude is induced in $C 2$ rotational symmetric unit cell whose amplitude can be modulated by tailoring the effective rotation degree. In addition to the lowest order rotational symmetry $(C 2)$, the discussion of higher order degrees of rotational symmetry, i.e. C3, C4, C6..., was performed in Ref. $70{ }^{70}$

With the emergence of cross-polarized component, we also discuss the asymmetric transmission and planar chirality from the aspect of circularly polarized light. The proposed $r$-SRR is demonstrated to function as an excellent quarter wave plate. Although the operation bandwidth is slightly broadened due to the excitation of dipole mode along both $x$ - and $y$-axes, a strategy to further broaden the operation bandwidth is required for applications in the broadband THz-TDS systems. One novel and efficient approach on the basis of concept of dispersion compensation will be discussed in next chapter where 
broadband operation of an ultrathin, flexible and robust quarter wave plate is designed. ${ }^{74,}$ 75 


\section{Chapter 4: Broadband polarization device}

Nowadays, the most widely applied polarizing components in optical systems mainly exploit the inherent birefringence in natural crystals such as liquid crystals and quartz where the phase delay is accumulated by engineering the thickness of the media. Such a kind of optical components is usually bulky and fragile, and operation wavelength is fixed once the thickness is finalized. The artificially engineered birefringence that is usually called form birefringence ${ }^{76}$ is obtained in stacked polymers, ${ }^{76}$ grating structures, ${ }^{77}$ piled up papers ${ }^{78}$ and anisotropic metamaterials. ${ }^{79}$ However, there always exist certain limitations in the previously reported approaches in designing optical components, such as limited operation wavelength, complicated fabrication procedure, high cost and low efficiency. The grating structure provides a simple way to engineer the birefringence by modulating the filling factors $(f)$, and the uniformly spaced wire-grid structures have found commercial applications in terahertz polarizers. In addition, the phase grating could be applied in many areas with the nullified zeroth-order diffraction intensity such as optical coatings in solar cells, ${ }^{80}$ optical modulators ${ }^{81}$ and beam splitters. ${ }^{82,83}$

\subsection{Introduction}

The key technique in phase grating is to effectively manipulate the zeroth-order diffraction efficiency that would be applied in many high-performance optical components. Diffractive effects play a key role if the periodicity $(p)$ of the grating is in the scale of wavelength while interacting with the incident light, however, the zeroth-order diffraction is no more 
dependent on the periodicity of the grating if the wavelength is much larger than the periodicity, i.e. $\lambda>>p$, at which case the effective medium theory applies. ${ }^{84}$ Here, we use the grating structure and engineer the phase delay originating from the artificially controlled filling factors in the subwavelength geometrical scale in terahertz wave. Through compensating the phase delay dispersion by combining two grating structures in the wave propagation direction, the proposed metadevice reveals the dispersion-free phase delay in a broad operational band, and thus enables function as an achromatic wave plate. The phase delay is also demonstrated to be modulated through rotating the metadevice along the propagation direction according to the space-variant Pancharatnam-Berry (P-B) phase concept with the dispersion-free features conserved. Such a metadevice is free-standing with the flexible polyimide as the spacer, which enables its ultrathin, extremely robust and flexible features for compact optical system and photonic applications.

\subsection{Theory and Discussion}

By employing the grating structure at the subwavelength scale, we are able to tailor the anisotropic response by engineering the filling factor $\left(f, f=d_{m} /\left(d_{a}+d_{m}\right)\right)$. The proposed grating is designed with period $p=120 \mu \mathrm{m}$ as schematically shown in Figure 4-1, and we probe the anisotropic transmission performance by linearly polarized light in the terahertz regime $(0.1-3.0 \mathrm{THz}, \lambda=300 \mu \mathrm{m}$ at $1.0 \mathrm{THz}) . d_{m}$ denotes the width of the metal strips whose effective refractive index is written as $n_{m}$, and $d_{a}$ is the width of the air part whose refractive index is $n_{\text {air }}=1$. The anisotropic zeroth-order effective refractive indices of the grating are calculated by $n_{T M}^{2}=\left((1-f) / n_{\text {air }}^{2}+f / n_{m}^{2}\right)^{-1}$ and $n_{T E}^{2}=(1-f) n_{\text {air }}^{2}+f n_{m}^{2}$ using 
the effective medium theory. In the terahertz regime, we simply regard the conductivity of metal (aluminum, $\mathrm{Al}$ ) as $\mathrm{DC}$ with a value of $3.72 \times 10^{7} \mathrm{~S} / \mathrm{m}$ which will provide a negative value of $\mathrm{n}^{2}$. Therefore, the transmission amplitude is reduced by increasing the filling factor where the square of effective refractive index is approaching to a negative value as shown in Figure 4-1(b) at $1.0 \mathrm{THz}$. The anisotropic grating configuration leads to a phase delay between the two orthogonally polarized components that are assigned to be parallel and perpendicular to the grating lines, respectively. Phase delay together with transmission amplitude along the two orthogonal components are engineered by tailoring the filling factors as shown in Figure 4-1(b) at $1.0 \mathrm{THz}$. By carefully designing the parameters of the grating, we could easily get the on-demand response for different applications, such as a quarter wave plate or a half wave plate. However, the phase delay of a single layer grating structure that is calculated by $\varphi_{d e l}=2 \pi t \Delta n / \lambda$ will always provide the very dispersive phase delay dependent on the operation wavelength, where $t$ is the thickness of the effective thickness of the metasurface, $\lambda$ is the wavelength and $\Delta n\left(\Delta n=n_{T E}-n_{T M}\right)$ is the refraction index difference between the two orthogonally polarized components. Therefore, it only provides an operation at a narrow band for the pre-designed functionalities, which reveals no obvious advantages to the crystal based optical components. 

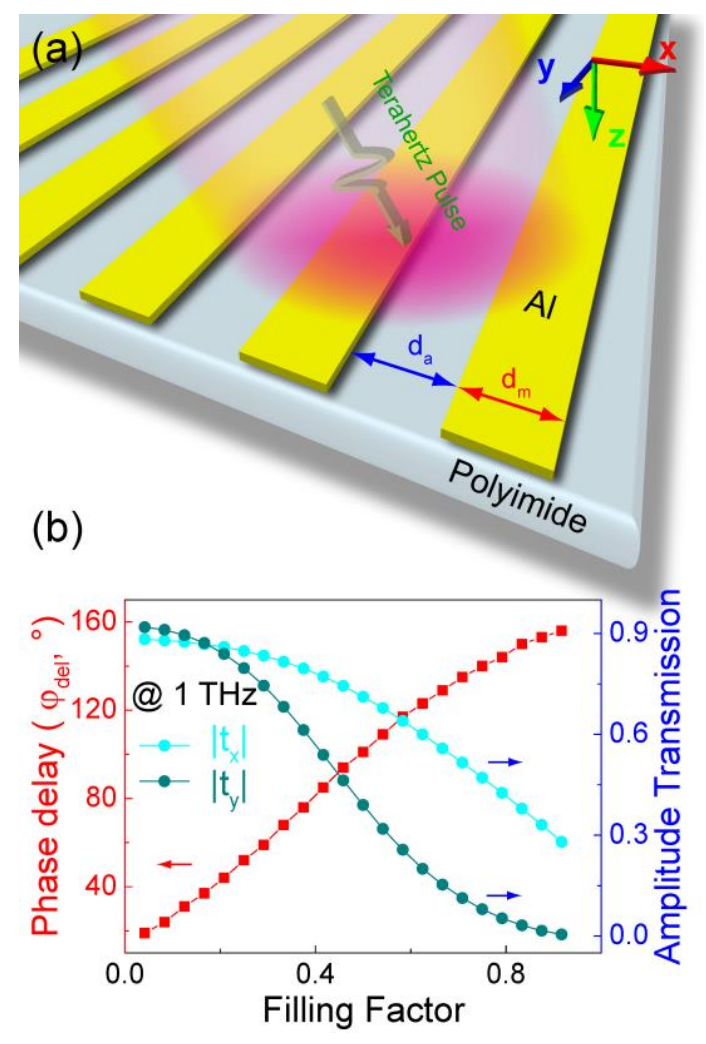

Figure 4-1. Transmission engineering of grating. (a) Schematic illustration of the grating structure on polyimide substrate in the terahertz regime. (b) Anisotropy engineering by tailoring the filling factors of the grating.

In order to obtain a broadband operation, we have to seek for an effective solution to compensate the dispersion of the phase delay. Since we are focusing on polarization control, we will investigate the phase delay and polarization conversion ratio (PCR) to present the phase and amplitude response, where $P C R=\left|\tilde{t}_{y}\right|^{2} /\left(\left|\tilde{t}_{y}\right|^{2}+\left|\tilde{t}_{x}\right|^{2}\right)$ with $\tilde{t}_{y}$ and $\tilde{t}_{x}$ describing the transmission components along $x$ and $y$ axes. The phase delay and PCR spectra are shown in Figure 4-2(b) for the proposed grating layer with filling factor $f \approx 0.5$ ( $d_{a}=62 \mu \mathrm{m}$ and $d_{m}=58 \mu \mathrm{m}$ ) that is illustrated in Figure 4-2(a). The grating layer is rotated by $45^{\circ}$ relative to the incident linearly polarized light in order to excite both co- and cross- 
polarized radiation in the transmission port. Based on the spectra of this single grating metasurface layer, we obtain a nearly dispersion-free PCR response for the orthogonal amplitude components, however, the phase delay reveals a dispersive feature according to the effective medium theory that approximately reveals a linearly dependence on the frequency. The phase delay dispersion exhibits a negative slope, and thus we can simply compensate this dispersion by adding another layer of metasurface providing a linear dispersion with positive slope as shown in Figure 4-2(c). This layer of grating is designed to have a filling factor of $f=0.08\left(d_{m}=14 \mu \mathrm{m}\right.$ and $\left.d_{a}=156 \mu \mathrm{m}\right)$ that is clockwisely rotated by $45^{\circ}$ relative to the first layer of metasurface, whose PCR and phase delay spectra are shown in Figure 4-2(d). We also observe the dispersion-free property of the PCR spectrum and a linearly dispersive phase delay spectrum, however, this second layer of grating provides the phase delay with a positive slope whose absolute value is similar to that of the first layer. Therefore, by simply combining these two layers of grating structures together with a dielectric spacer isolating them, we get a dispersion-free phase delay response as shown in Figure 4-2(e). The combined transmission spectra are presented in Figure 4-2(f) where both phase delay and PCR manifest the dispersion-free performance in a broad frequency band ranging from $0.95 \mathrm{THz}$ to $1.30 \mathrm{THz}$.

In this dispersion compensation mechanism, we ignore the longitudinal coupling and Fabry-Perot (F-P) effect between these two layers. Here the F-P effect is negligible due to the very low filling factors of the second layer metasurface which reflects the wave at an extremely low efficiency. Weak longitudinal coupling effect should be considered while 
designing the spacer thickness in order to optimize the operation bandwidth and value of phase delay.
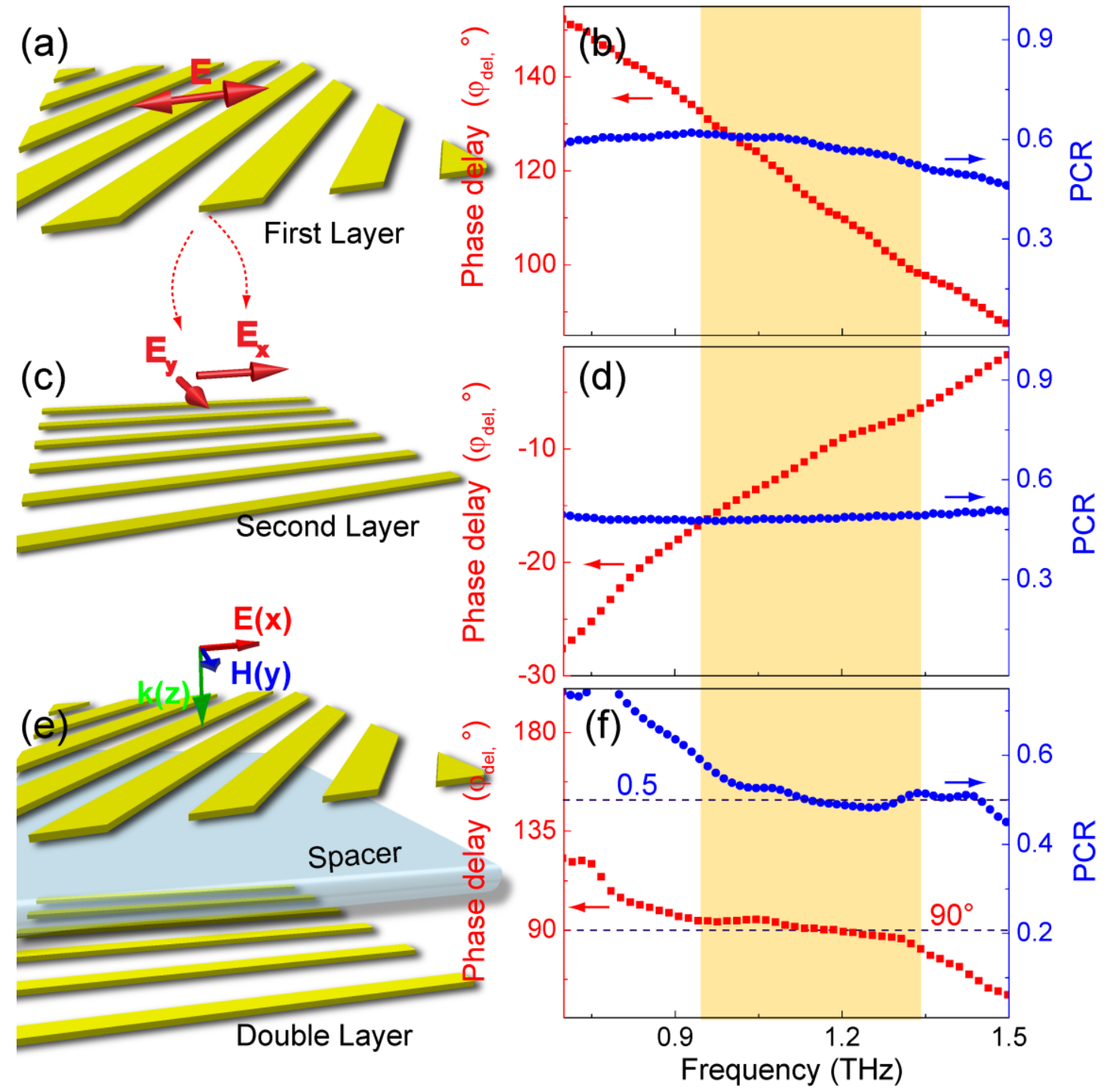

Figure 4-2. Approach of dispersion compensation with two-layer metasurfaces. (a) The

first layer of grating structure with (b) the transmission spectra; (c) the second layer of grating structure with (d) the transmission spectra; and (e) integrated metadevice with twolayer grating structure that shows (f) the dispersion-free spectra. 
Detailed transmission amplitude and phase delay of two orthogonal components in both experiments and simulations are presented in Figure 4-3, where we observe large transmission amplitude in the interested frequency range. With overall transmission amplitude larger than 0.5 for both co- and cross-polarization components, polarization conversion efficiency reaches more than $50 \%$ with an excellent polarization state quality. Due to the limitation of experimental setup, we only perform the transmission measurements, which is enough to test the performance of the proposed metadevice.

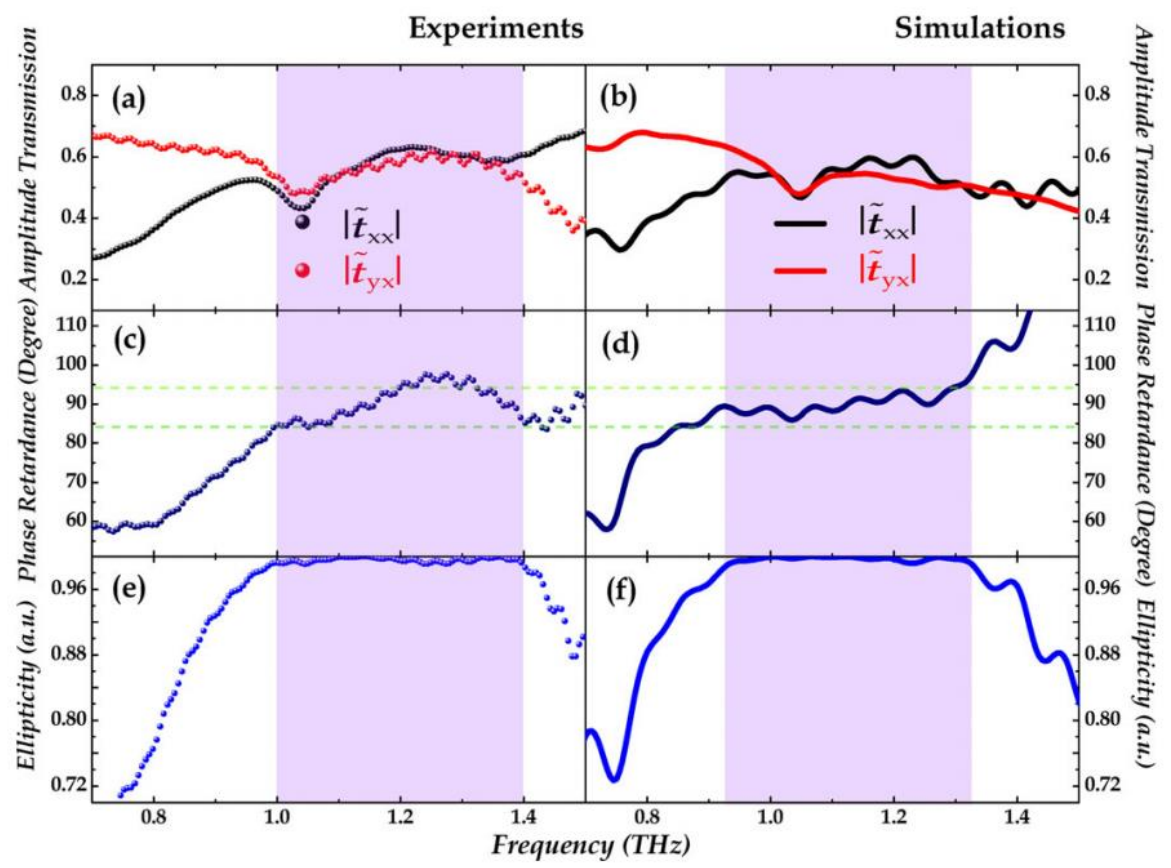

Figure 4-3. Measured and simulated transmission amplitude, phase response and ellipticity of two orthogonal polarization components.

\subsection{Tunable phase delay by $P-B$ phase}

We aim at a broadband quarter wave plate (QWP) that requires the phase delay to be $90^{\circ}$ and PCR to be 0.5 . With accurately designed parameters of the grating structures, we 
obtained the required values in a broadband regime as shown in Figure 4-3(e), and thus a dispersion-free QWP was achieved that operates in a broad bandwidth ranging from 0.95 to $1.30 \mathrm{THz}$. With the pre-designed phase delay, the functionality of the metadevice is fixed, and in order to find an approach to tune the response, we here introduce the concept of space-variant Pancharatnam-Berry (P-B) phase. The P-B phase is simply interpreted from the Poincare sphere where the complex Jones vectors are mapped on the surface of the sphere represented by the Stokes parameters as shown in Figure 4-4. As we have discussed in Chapter 2, the surface of the Poincare sphere covers all the possible polarization states using a most straightforward way and explicitly exhibits the trajectory of polarization modulation. We consider a $90^{\circ}$ polarization rotation from linearly horizontally polarized state (LHP, defined along $x$-axis) to linearly vertically polarized state (LVP, defined along $y$-axis) which is the so-called cross-polarized transformation corresponding to the element $\tilde{t}_{y x}$ in the Jones matrix. The conversion trajectory is visualized as curve 1 mapped on the surface of the sphere as shown in Figure 4-4(b). However, there would be infinite possible trajectories to connect points $i$ and $j$ that indicate the paths to realize this cross-polarization transformation. In fact, the cross-polarized element could be represented as $e^{-i \beta \hat{\sigma}_{3}} \tilde{t}_{y x} e^{i \beta \hat{\sigma}_{3}}$ where $\hat{\sigma}_{3}$ is the Pauli matrix in the space rotated frame and $\beta$ is the rotation angle of the medium that functions as a polarization rotator. This general cross-polarized transforming expression covers all the possible trajectories from point " $i$ " to " $j$ " when angle $\beta$ varies from 0 to $360^{\circ}$. Although all the possible trajectories indicate the same polarization rotation behavior, the area covered by the two different possible trajectories as shown in Figure 44(b) by curve 1 and curve 2 on the surface represents the relative phase difference between 
the final states along these two trajectories, which is the so-called P-B phase. For simplicity, the P-B phase introduces the space-variant angle $\beta$ into the phase item of the cross-polarized components in the Jones matrix.
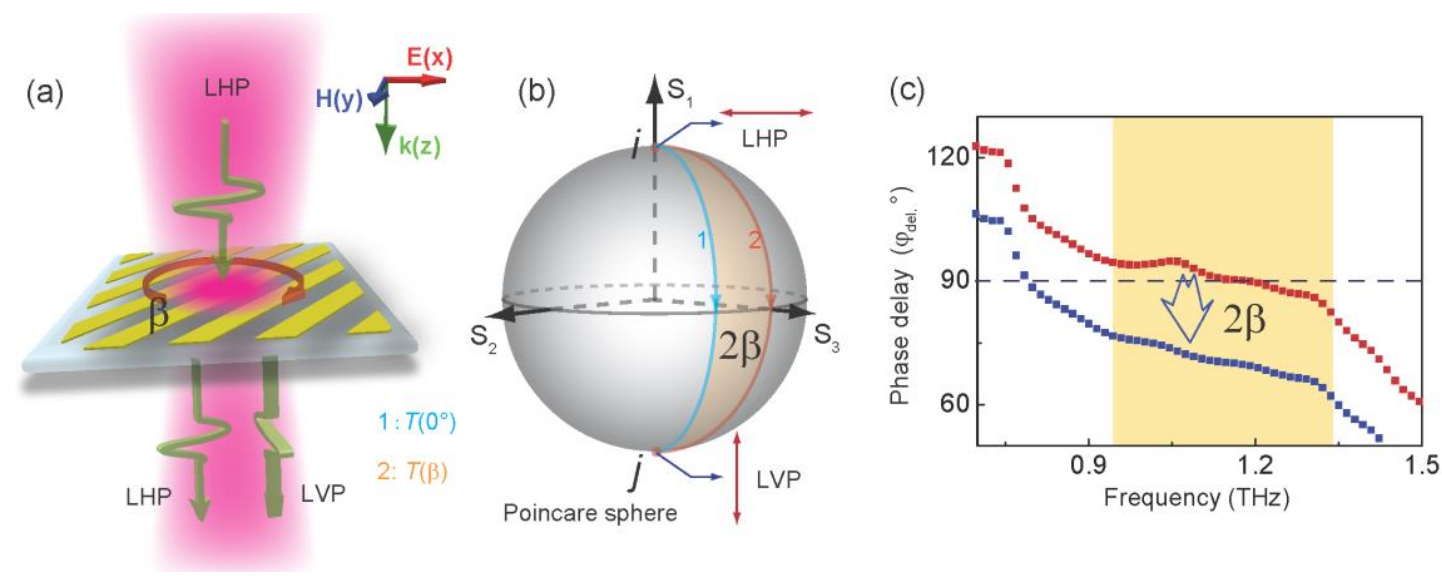

Figure 4-4. P-B phase interpretation and application. (a) Schematic diagram of the transmission behavior of anisotropic grating. (b) The P-B phase interpretation using Poincare sphere. (c) Application of P-B phase on the proposed metadevice.

The P-B phase is indeed possible to be applied to the dispersion-free metadevice by simply rotating the sample so that the phase response of the cross-polarized components is modulated. The dispersive slope of phase delay for each single layer of grating is conserved which renders the dispersion-free features of the metadevice unchanged. Through rotating the metadevice along the propagation direction, the transmitted co-polarized component is unchanged while the cross-polarized components are modulated in the phase item by a value of $2 \beta$. Therefore, the resultant phase delay along the orthogonal components in the far field will be modulated by a value of $2 \beta$. In order to verify this theory, we performed the numerical simulations using CST Microwave Studio in time-domain solver by rotating 
incident linearly polarized light by an angle of $\beta$ where $\beta$ is positive for the counterclockwise rotation. Simulated phase delay results are illustrated in Figure 4-4(c) with $\beta=10^{\circ}$ where the parallel shift of around $20^{\circ}$ with the dispersion-free spectra are observed that indicates the tuning of the phase delay and the preservation of the dispersionfree feature.

\subsection{Experimental results}

Conventional photolithography technique was employed to perform the fabrication of the two-layer metadevice. The spacer layer is isotropic polyimide $\left(\varepsilon_{\text {poly }}=2.96+0.27 i\right)$ that is transparent for visible light, and thus the accurate alignment between the two layer metasurfaces becomes easy. The fabricated sample is shown in Figure 4-5 where the thickness of space is $t=22 \mu \mathrm{m}$ and that of the metal aluminum is $200 \mathrm{~nm}$ with two more layers of $22 \mu \mathrm{m}$ thick polyimide capping on top and bottom of the metal grating in order to protect the metal structure and enhance the robustness of the metadevice. The total thickness of the entire metadevice is estimated to be around $70 \mu \mathrm{m}$, and it is extremely flexible without substrate as shown in Figure 4-5 (a) and (b). Such an ultrathin, robust and flexible metadevice will be easily integrated into a compact optical system and find industrial applications in optical communication and display. More detailed tests of the sample performance will be discussed in the next subsection. 
(a)

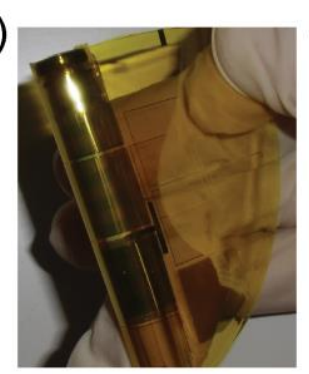

(b)

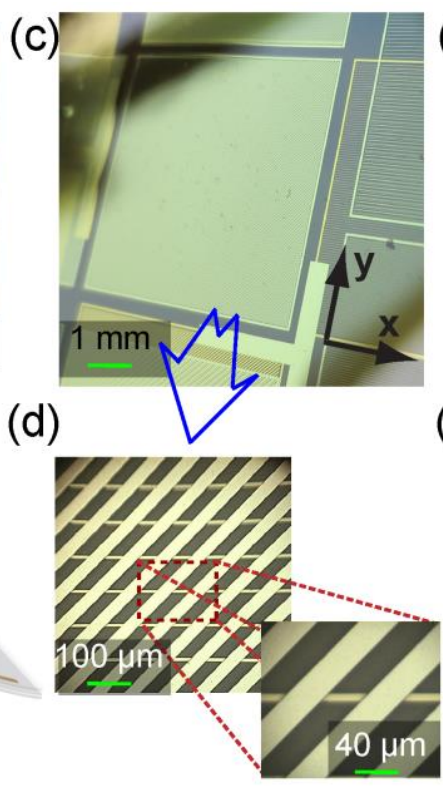

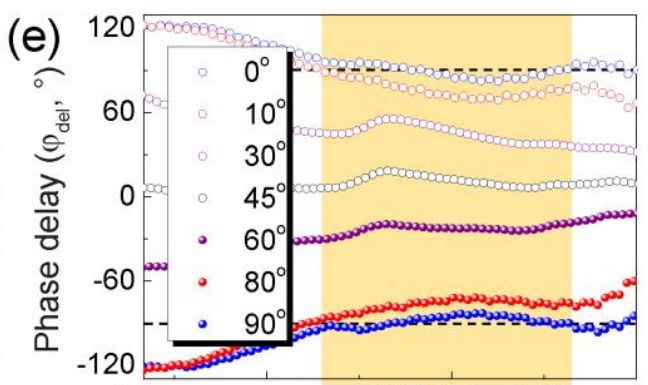

(f)

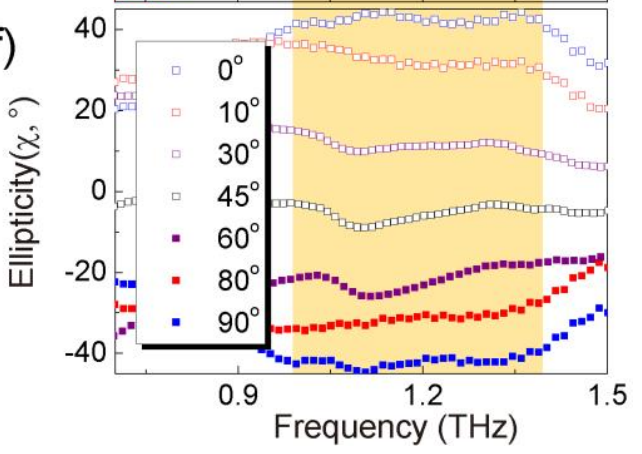

Figure 4-5. Fabricated sample and measured transmission response. (a) Image to test the flexibility of the sample and (b) schematic illustration of the flexible metadevice. (c) Image of the fabricated metadevice with (d) zoomed microscopic images of the grating structures. (e) Phase delay response of the metadevice and (f) calculated ellipticity of the output light.

A fiber-based 8-f terahertz time-domain spectroscopy system (THz-TDS) whose spectrum bandwidth is from 0.2 to $2.5 \mathrm{THz}$ and beam waist diameter is around $3 \mathrm{~mm}$ was used to measure the transmission behavior of the fabricated metadevice. The transmission signals along two orthogonally polarized directions were both coherently measured using the setup as discussed in the experimental section, and normalized to the co-polarized signals of dry air. The frequency domain data were extracted with both amplitude and phase information as shown in Figure 4-5(e) and 4-5(f). We firstly look at the spectra while $\beta=0^{\circ}$ at the initial state for the metadevice that is designed to operate as a broadband QWP. The phase delay 
reveals an approximately flat response in the frequency range from 1.0 to $1.4 \mathrm{THz}$ that indicates the broadband dispersion-free operation, and the value is around $90^{\circ}$ that meets the requirements of a QWP. In order to exhibit the polarization transformation performance of the metadevice, ellipticity spectra are also shown in Figure 4-5(f) where the ellipticity is constant at around $45^{\circ}$. This further demonstrates the evenly distributed polarization state in a broadband frequency regime for the metadevice operating as a dispersion-free QWP.

While applying the P-B phase by rotating the metadevice relative to the wave propagation direction, we also probe the modulation of the phase delay in experiments. From Figure 45(e) and 4-5(f), it is clearly observed that the phase delay is modulated by $-2 \beta$ as the metadevice is rotated by an angle $\beta$, and the dispersion-free feature is still preserved in the interested bandwidth as the shaded area shown in the graph. We note that it is the metadevice that is rotated in order to introduce the space-variant polarization angle in experiments. With the modulation of phase delay, the output polarization state from the metadevice is thus changed. This is represented by the calculated ellipticity spectra where the polarization state is continuously tuned from RCP to LVP and then to LCP. The measured amplitude of both co- and cross-polarized components are higher than 0.5 which enables the metadevice to operate with transmission efficiency higher than $50 \%$ in the discussed frequency band. Thus far, the concept of dispersion compensation has been verified through numerical simulations and accurate measurements; and the tunability of the output polarization states by applying the P-B phase is also demonstrated. 
(a)

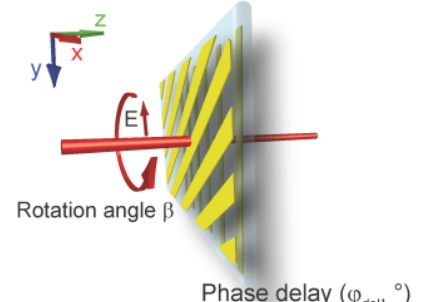

(b)

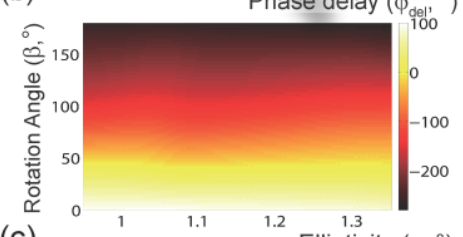

(c)

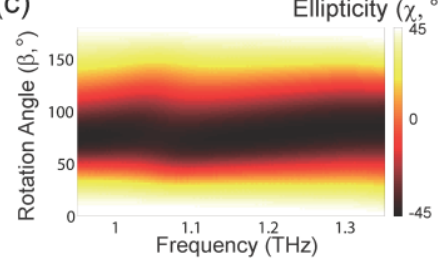

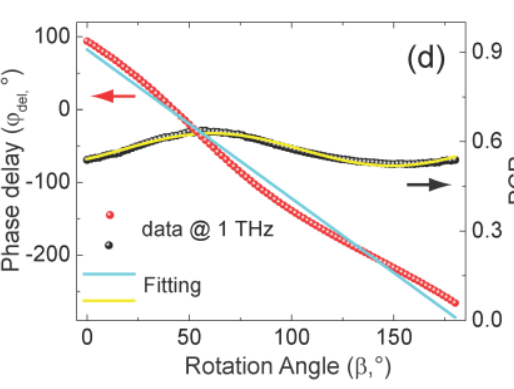
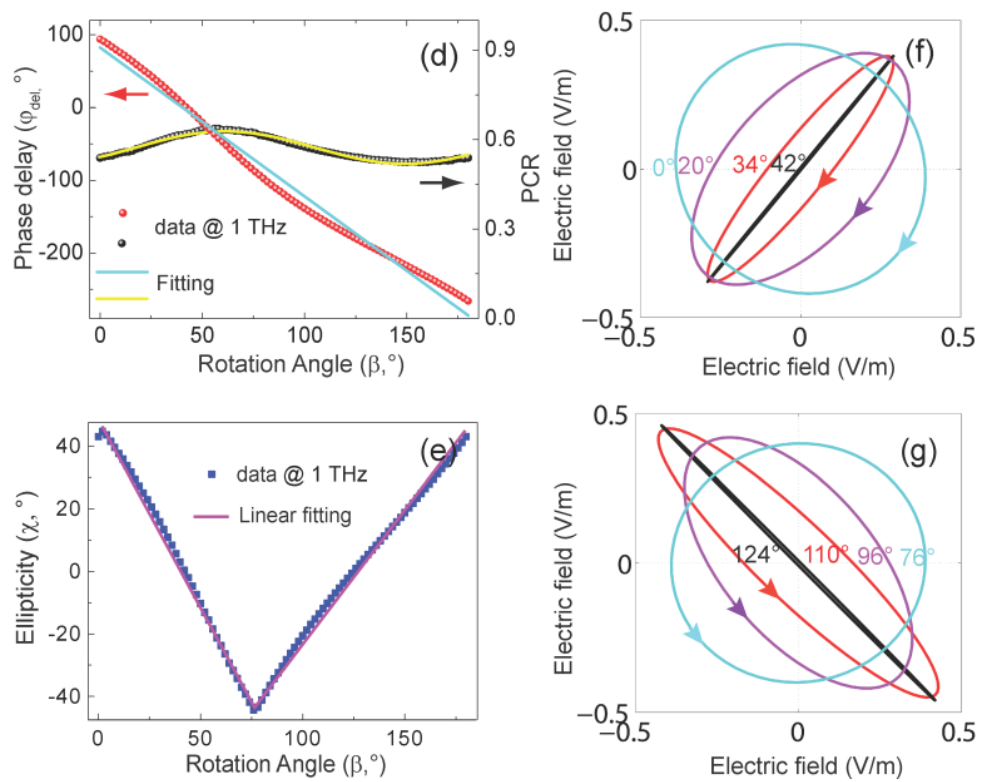

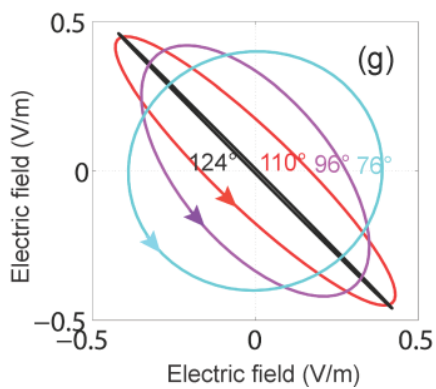

Figure 4-6. Investigation of the metadevice performance in details. (a) Schematic diagram of the metadevice. (b) Phase delay and (c) ellipticity spectra of output light relative to the rotation angle of the metadevice. (d) The analysis of phase delay, PCR and (e) ellipticity with respect to the rotation angle of the metadevice. (f) Several representative states of right-handed elliptically polarized light and (g) left-handed elliptically polarized light modulated by rotating the metadevice at $1.0 \mathrm{THz}$.

Detailed evolution of phase delay and ellipticity spectra are shown in Figure 4-6(b) and 46(c) by rotating the linearly polarized vector in steps of $2^{\circ}$ from $0^{\circ}$ to $180^{\circ}$ range where the dispersion-free features of both phase delay and ellipticity are preserved in the dynamic process. In addition, the spectra also reveal a continuous modulation that is in line with PB phase prediction. For visualizing the modulation features relative to the angular rotation of the metadevice, we exhibit the extracted data of phase delay, PCR and ellipticity at 1.0 THz, and investigate the relationship in details as shown in Figure 4-6(d) and 4-6(e). The 
phase delay reveals a linear dependence on the rotation angle $\beta$ varying from $90^{\circ}$ to $-270^{\circ}$ by rotating the metadevice from $0^{\circ}$ to $180^{\circ}$. The behavior follows the linear equation $\varphi_{d e l}=-2 \beta+90^{\circ}\left(0^{\circ} \leq \beta<180^{\circ}\right)$ which matches with the theoretical P-B phase. As for PCR that determines the angle of polarization ellipse (AOP), there is a slight fluctuation in the amplitude from 0.45 to 0.55 following a sinusoidal profile fitted using equation $\mathrm{PCR}=0.5+0.05 \sin ^{2} \beta$. This fluctuation of PCR will not affect the AOP obviously so that the major axis of the polarization ellipse of output light will always approximately orient along $45^{\circ}$ or $135^{\circ}$. The ellipticity $(\chi)$ spectra numerically present the output polarization ellipse defined as $\sin 2 \chi=S_{3} / S_{0}=\left(2\left|t_{x}\right|\left|t_{y}\right| \sin \varphi_{\text {del }}\right) /\left(\left|t_{x}\right|^{2}+\left|t_{y}\right|^{2}\right)$. By estimating PCR as 0.5 , we get $\sin 2 \chi \propto \sin \varphi_{d e l}$ and the ellipticity value also reveals a linear dependence on the rotation angle as observed in Figure 4-6(e). The polarization ellipticities are modulated from $45^{\circ}$ to $0^{\circ}$ to $-45^{\circ}$ and then go back to $0^{\circ}$ and $45^{\circ}$ as the metadevice is rotated from $0^{\circ}$ to $180^{\circ}$. Corresponding polarization states are schematically illustrated in Figure 4-6(f) and 4-6(g) at several representative metadevice angles where the major axes of the polarization ellipse always orient along $45^{\circ}$ or $135^{\circ}$ direction. 


\subsection{Metadevice tests}
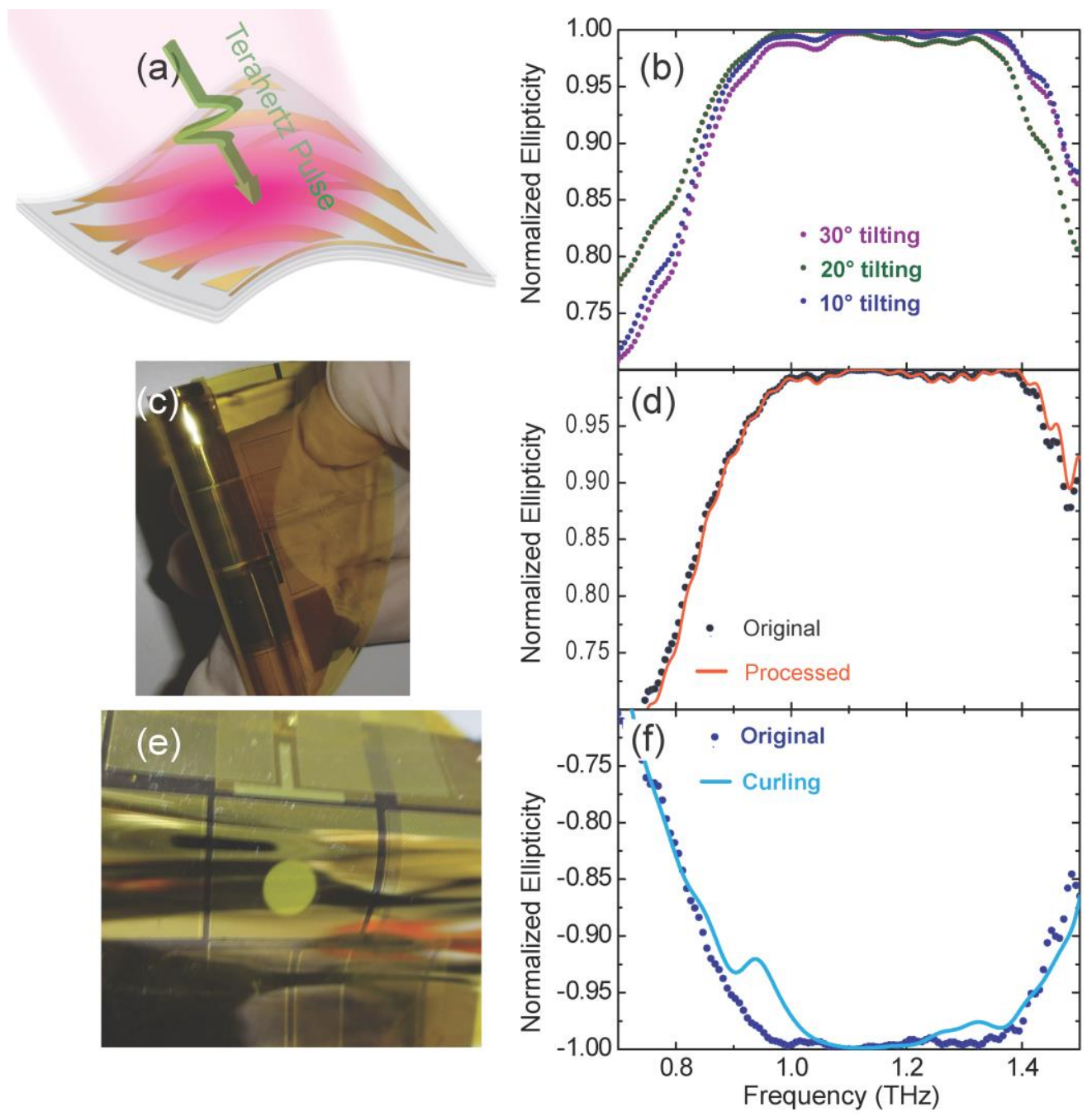

Figure 4-7. Tests of the metadevice on the flexibility and stability performance. (a)

Schematic illustration of the tilted incidence on the flexible metadevice; (b) measured results of ellipticity with tilted incidence. (c) Image of flexible sample with (d) ellipticity of the metadevice after processing with mechanical force. (e) State of curved sample and the corresponding (f) ellipticity spectra.

Since the metadevice consists of three layers of polyimide isolators and two layers of grating structures without substrate, it is ultrathin with total thickness around $70 \mu \mathrm{m}$, and it 
also exhibits excellent flexibility. In addition to these features, we also test the performance of the metadevice at diverse operational conditions. First, the metadevice is insensitive to the angle of the incident wave, and the performance operating as a broadband QWP is almost stable with the incident angle tilted as large as $30^{\circ}$ as shwon in Figure 4-7(a) and 47(b). Ellipticity spectra show no obvious modulation when the incident wave vector angle is tilted to $10^{\circ}$ and $30^{\circ}$. Secondly, with the protection of polyimide layer, the metadevice reveals an anticorrosion feature that will effectively extend the lifetime of the device and enable the possibility to operate in different harsh environments. We also test the performance of the metadevice after twisting it randomly with considerable mechanical force in order to check the robustness. The physical configuration is demonstrated intact without obvious damage whose performance as a QWP is still perfect with ellipticity spectra as shown in Figure 4-7(d), suggesting the excellent stability and robustness. In addition, the performance stability is proved in Figure 4-7(e) and 4-7(f) where the response was measured when the metadevice was in a curved state, and the ellipticity spectra reveal slight modulation with the overall functionality unaffected.

\subsection{Conclusions}

In this chapter, we propose a simple but novel and effective approach to obtain the broadband operation of a quarter wave plate by the concept of dispersion compensation through integrating two metasurfaces. The two metasurfaces provide opposite dispersion slopes of phase delay, and a simple addition of them provides a compensated dispersion. 
We note that although Fabry-Perot (F-P) effect in a multilayer metasurface improves the transmission efficiency ${ }^{73}$, we diminish it in order to apply the simple addition of dispersion compensation for a broadband operation. Such a dispersion compensation concept is also applicable in different frequency bands for the broadband and achromatic photonic devices so that the metadevice is adjustable by scaling the parameters of the grating and spacer thickness accurately. The dispersion-free phase delay is also demonstrated tunable with the space-variant P-B phase concept that transfers the spatial rotation angle into the phase item of the cross-polarized components. Taking advantage of the P-B phase, the metadevice converts the polarization state into any desired states by spatial rotation of the device. The proposed metadevice design also exhibits the extremely flexible, robust and stable performance, and has great potentials for applications in industrial optical facilities and devices such as polarization modulator and multiplexer in polarization-division multiplexing technique in the communication systems.

In the dispersion compensation concept proposed in this chapter, we ignore the longitudinal coupling effects between the two metasurfaces which is weak in this system so that we can assume a simple addition of the phase responses in the two single metasurfaces. In fact, coupling effects in metamaterial system play extremely important roles in engineering their functionalities. In order to clearly investigate the coupling mechanism in the metamaterial array, two different lateral near-field coupling schemes will be discussed in the next two chapters in details. 


\section{Chapter 5: Intra coupling switch in polarization control}

The longitudinal coupling effect affects the dispersion compensation effects which is related to the spacer properties. In the past decades, near-field coupling effects have been a research hot spot in the metamaterial community with the artificially designed flexibility of the building blocks. ${ }^{85-92}$ Diverse intriguing phenomena were reported that were reproduced or mimicked by carefully designing the unit cell in the metamaterial array, such as the analog of electromagnetic induced transparency (EIT) $)^{86,93-97}$ or absorption (EIA) $)^{98}$ and excitation of Fano lineshape resonances. ${ }^{12,}{ }^{99-103}$ Among all these studies, split-ring resonator (SRR) was the most commonly employed building block as a meta-atom in the microwave and terahertz frequency regimes. ${ }^{9}, 104,105$ More complex coupled meta-molecule consisting of several meta-atoms in one unit cell was designed for different functionalities and spectral features. ${ }^{85,}$, 7, 106-108 In the coupled meta-molecular system, bright and dark modes are defined based on the loss contrast of the resonators or the direct coupling to external field in the study of EIT analogue, however, effects of near-field coupling in the polarization control area have not been clearly discussed. In this chapter, we will focus on the near-field coupling effects on polarization control, and provide a new perspective on the switch of the near-field coupling channels.

\subsection{Experimental design}

The near-field coupling has been probed in Ref. $85^{85}$ where the coupling strength defined from mode splitting depends on the geometrical distance between the neighboring metaatoms. Employing the same configuration as Ref. 85 where two orthogonally orientated 
SRRs are constructed as one meta-molecule, we here study the angular dependence of nearfield coupling effects by rotating the meta-atoms without adjusting the geometrical distance between the neighboring meta-atoms.
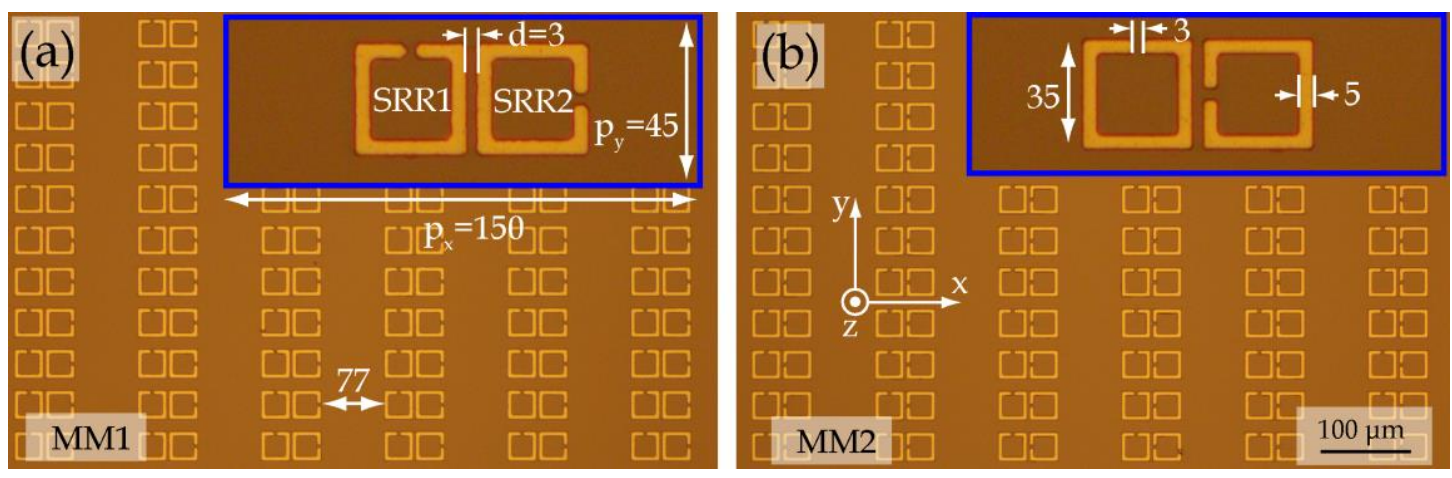

Figure 5-1. Experimental design. Microscopic images of fabricated samples (a) metamaterial 1 (MM1) and (b) metamaterial 2 (MM2).

In order to probe the angular dependence of near-field coupling in the double SRR metamolecular system, we fabricated two representative samples using conventional photolithography technique with sample images as shown in Figure 5-1, where all the parameters are identical except the $180^{\circ}$ rotation of SRR2. We define the two meta-atoms in one meta-molecule as SRR1 (left) and SRR2 (right), respectively, and the two configurations of meta-molecules are designated as MM1 and MM2, respectively. SRR1 and SRR2 are arranged orthogonally so that only one meta-atom is coupled to external linearly polarized light and excited the fundamental resonance mode that is defined as a "bright" meta-atom. The other meta-atom is only accessible to the excitation of fundamental mode by near-field coupling to the neighboring bright meta-atom that is called a "dark" resonator. Here the near-field coupling between the neighboring meta-atom inside 
the meta-molecule is named as intra coupling. According to the understanding of coupling strength dependence on the geometrical distance, SRR1 and SRR2 are separated by $d=3$ $\mu \mathrm{m}$ in order to guarantee the operation within the strong coupling regime for MM1. The angular dependence coupling is probed through rotating SRR2 by $180^{\circ}$ in MM2. All the detailed geometrical parameters are indicated in Figure 5-1, and the metamaterial structures are made of aluminum on a double polished high-resistivity silicon.

\subsection{Results and discussion}

The responses of samples are probed by a THz-TDS system with wire-grid polarizers inserted to coherently obtain the co- and cross-polarized transmission signals. It is known that the co-polarized transmission spectra of MM1 in the strong coupling regime reveal a large mode splitting, and this is verified from the experimental data in blue dash lines as shown in Figure 5-2. Due to the rectangular lattice of the unit cell, it exhibits an anisotropic response of the mode splitting spectra with $x$-polarized and $y$-polarized incidence by comparing the spectra in Figure 5-2(a) and (b). However, no mode splitting is observed in the transmission spectra of MM2 with SRR2 rotated by $180^{\circ}$ as the red lines shown that indicates the cease of the intra coupling in the strong coupling regime. 


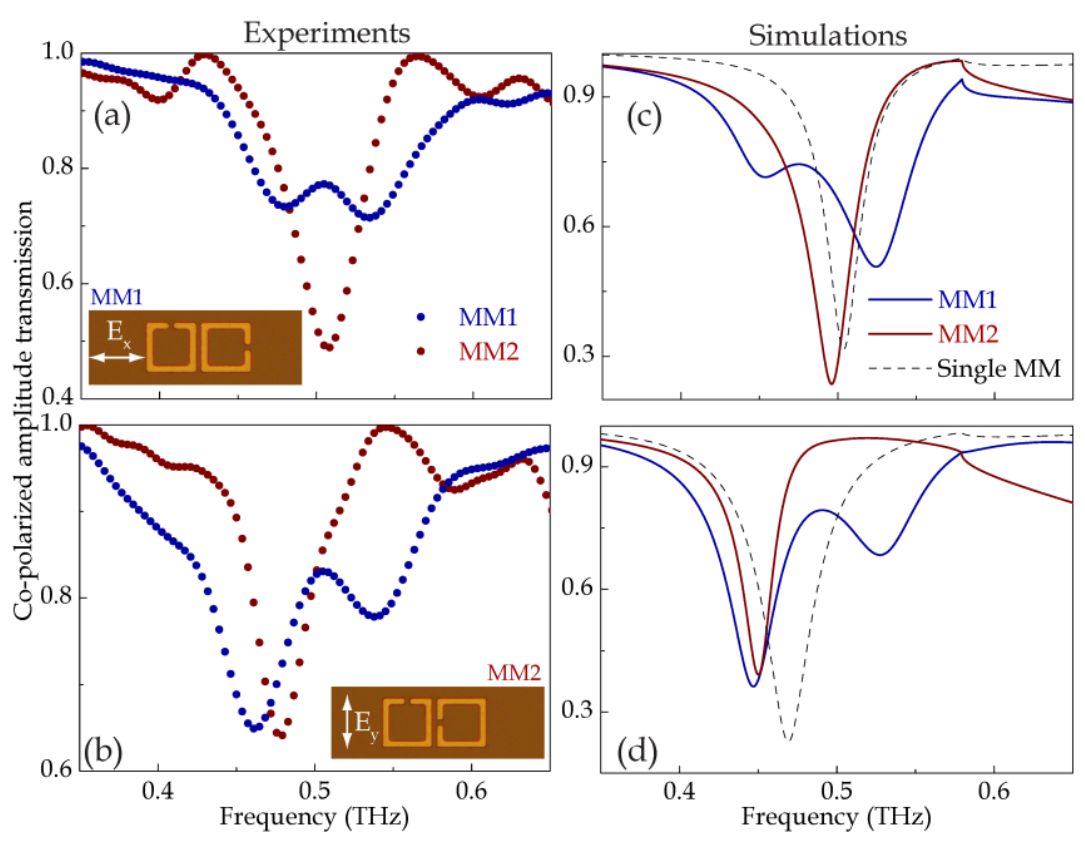

Figure 5-2. Measured and simulated transmission spectra of MM1 and MM2. (a) and

(b) measured spectra of the two samples with $x$-polarized and $y$-polarized incidence, respectively. (c) and (d) Corresponding simulated spectra with the eigen resonance spectra of a single bright meta-atom array on the identical lattice.

The mode splitting spectra in the coupled system are commonly known that the bright SRR1 with direct excitation of $x$-polarized incidence will induce the resonance of SRR2 through inductive coupling. Surprisingly, the inductive channel is switched off according to the co-polarized transmission spectra for MM2, which indicates the angular dependence of the intra coupling. A similar angular dependence intra coupling switch phenomenon is observed from the spectra with $y$-polarized incidence where SRR2 acts as the bright metaatom. To further validate the results and obtain an in-depth understanding, simulations were performed using frequency domain solver of CST Microwave Studio by building the models with the identical configurations of experimental samples, and the results are 
exhibited in Figure 5-2(c) and (d). We observe a good match between the measured and simulated results, and the very fine spectral feathers are also captured by the high-resolution and accurate simulation tool. Simulation also provides a freedom to detect the angular dependence coupling switch phenomenon in more details. We first perform simulations of a single bright meta-atom on the identical lattice so that we obtain the eigen resonance spectra of the bright resonator array as the dash lines shown in Figure 5-2(c) and 5-2(d). By comparing the spectra properties of MM2 and the corresponding single bright resonator array, they exhibit very slight deviation with each other in resonance frequency and line width. This indicates the extremely weak influence of the neighboring dark meta-atom on the bright one in consequence of the cease of inductive coupling channel. Therefore, the dark meta-atom in MM2 becomes almost invisible to the incident light in terms of the spectral signature.

In addition to the mode splitting in co-polarized spectra that reflect the intra coupling phenomenon, cross-polarized spectra also exhibit a direct measure of the coupling strength since it is the dark meta-atom that radiates the cross-polarized component to the far field as schematically shown in Figure 5-3(a). This is verified by the strong measured as well as simulated cross-polarized amplitude as the blue lines shown in Figure 5-3(b) and 5-3(c) for MM1. However, extremely weak cross-polarized signals are observed in both measurements and simulations for MM2 as the red lines shown in the graph. This result clearly demonstrates the switch on and off of intra coupling due to angular rotation of metaatoms from the obvious contrast of cross-polarized signals. The slight disagreements 
between measured and simulated data root in the limited scan time, low signal to noise ratio of the experimental system and limited extinction ratio of polarizers, especially for the case of MM2 whose cross-polarized signals are too low to be accurately detected in the experimental system.
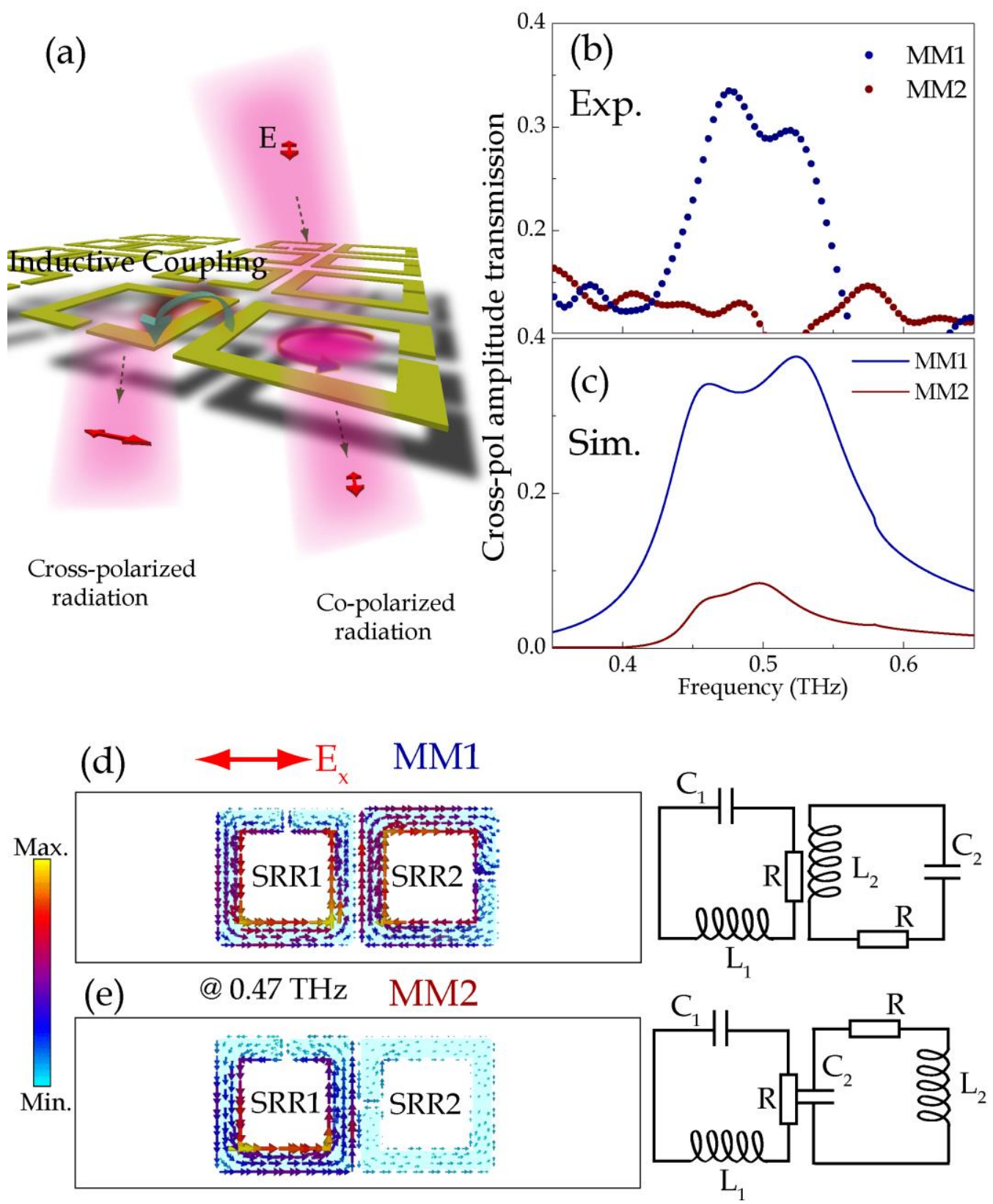

Figure 5-3. Cross-polarized spectra and understanding of angle-dependent intra coupling switch. (a) Schematic diagram of the coupled metamaterial array to induce the 
cross-polarized component. (b) and (c) The cross-polarized transmission amplitude in experiments and simulations, respectively. (d) and (e) Simulated surface current distributions of MM1 and MM2 with the corresponding equivalent circuit models.

Far-field spectral properties originate from the local resonance mode excitation of the resonator that reflects in the electromagnetic field distributions on the resonators. As shown in Figure 5-3(d) and 5-3(e), surface current distributions at $0.47 \mathrm{THz}$ are visualized where SRR1 behaves as the bright meta-atom and SRR2 is the dark one with $x$-polarized excitation. We can observe the current loop on SRR1 forming a magnetic dipole, and a similar current loop is also formed in the neighboring dark SRR2 that indicates the occurrence of energy transfer through near-field intra coupling by magnetic dipole interaction. The mutual interaction between the two excited meta-atoms forms two resonant energy states that finally results in a mode splitting spectrum. When we probe the current distributions in MM2, SRR1 is still excited with the current loop behaving as a bright meta-atom, however, there is almost no current flowing on the surface of SRR2 which indicates neither coupling to external field nor intra coupling to SRR1. Therefore, SRR2 reveals an extremely weak interaction to the bright SRR1 which thus results in a negligible spectral influence in the far field.

The intra coupling is interpreted as the inductive interaction through the magnetic dipoles due to the bianisotropic property of SRR. Here, the induced electromotive force in an equivalent circuit of the resonator should be proportional to the changing rate of the 
magnetic flux penetrating through the circuit according to the Faraday's law. In this work, all the geometrical configurations of resonators are kept the same between MM1 and MM2 except the orientation of SRR2 so that the area of SRR2 and the excitation of bright SRR1 is identical between MM1 and MM2, which thus guarantees the unchanged magnetic flux in the area of SRR2. However, the two systems reveal a non-intuitive angle-dependent switch of intra coupling contradictory to the ordinary understanding, which has been demonstrated by the far-field co-polarized mode splitting spectra, strength of crosspolarized signals and the near-field surface current distributions from both experiments and simulations.

The equivalent circuit model ${ }^{109,110}$ is employed to provide an understanding of this nonintuitive intra coupling switch by regarding the split gap of a SRR as an equivalent capacitor $(C)$ and the continuous lossy wire as an equivalent inductor $(L)$ with a resistor $(R)$ as shown in Figure 5-3(d) and 5-3(e). For a single gap SRR, there is no rotational symmetry existing, and thus locations of the equivalent components play the significant roles in determining the intra coupling strength. At the fundamental resonance mode, the magnetic dipole interacts with the dark meta-atoms by inductive coupling through the equivalent inductor in the equivalent circuit model. Considering the mirror symmetry of SRR, the equivalent inductor should be located opposite to the equivalent capacitor in the square circuit. We notice that the nearest distance between the equivalent inductors in the neighboring circuit models is different between MM1 and MM2. Therefore, the stronger coupling occurs in MM1 with a smaller coupling distance than that in MM2 that has the 
larger coupling distance. Although the mutual inductive coupling mainly depends on the locations of equivalent inductors, the equivalent capacitors also matter in the overall spectral profiles in determining the radiative strength and eigen resonant frequency.

\subsection{Application in polarization switch}

Far-field cross-polarized component induced by near-field inductive coupling always accompanies with a phase lag in the oscillation relative to the co-polarized radiation on the basis of the Faraday's law. As the inset graph shown in Figure 5-4(a), such a lag of crosspolarized oscillation was captured by the coherent time-domain signals. Such a delay provides an alternative to harvest the phase delay by the ultrathin metasurface that is beyond the bulk birefringent crystals. In order to numerically investigate the phase delay and the resultant polarization modulation performance by the coupled metasurface, we calculated the Stokes parameters of the output light, and obtained the angle of polarization ellipse (AOP) and ellipticities as revealed in Figure 5-4.
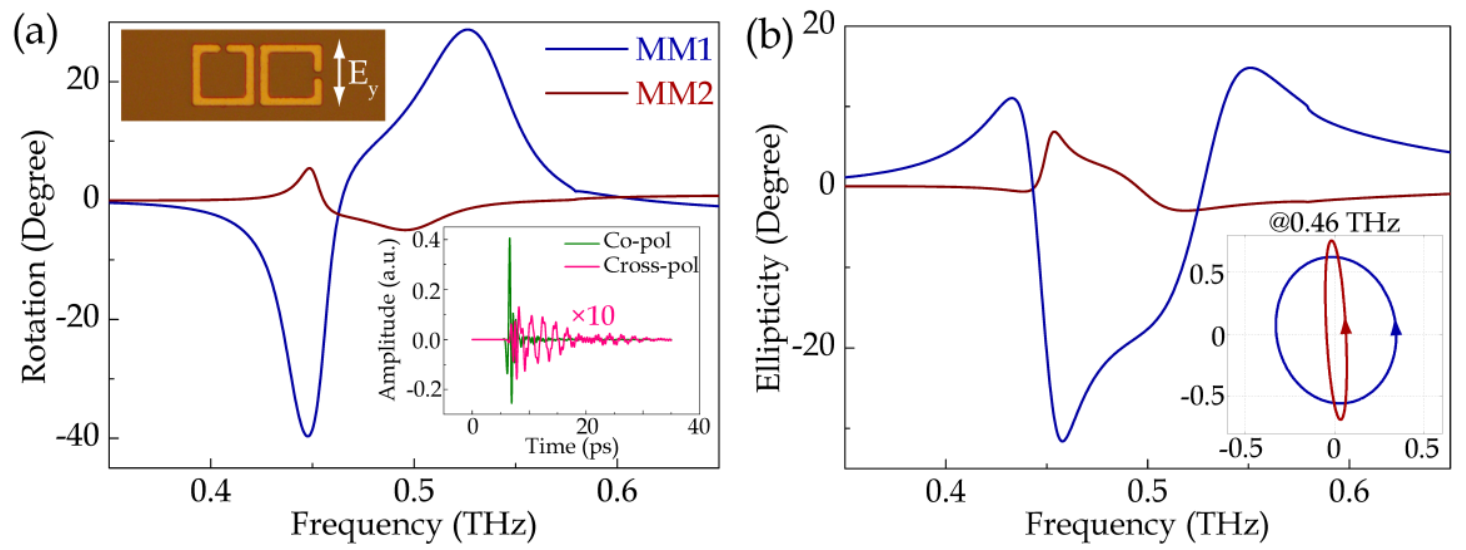

Figure 5-4. Polarization modulation performance of the metasurface. (a) Rotation degree of polarization ellipse of output light for MM1 and MM2 with insets of sample image an time-domain signals for co- and cross-polarized components. (b) Ellipticities of 
output light with insets of polarization states at $0.46 \mathrm{THz}$.

From Figure 5-4, we observe the obvious spectral contrast of AOP and ellipticity of output light between MM1 and MM2. For MM1, it shows around $-40^{\circ}$ rotation of polarization ellipse at $0.44 \mathrm{THz}$ due to the emergence of cross-polarized component at which frequency the ellipticity is almost $0^{\circ}$. This indicates a strong optical activity induced by the coupled metasurface MM1 whose main axis is rotated by around $-40^{\circ}$ with $y$-polarized incidence. However, we are unable to observe obvious modulation of AOP from MM2, and thus no optical activity exists in MM2. Similarly, the modulation of polarization ellipticity is also contrasting between output lights from MM1 and MM2, where the ellipticity degree reaches to $-30^{\circ}$ for MM1 at $0.46 \mathrm{THz}$ while it is $0^{\circ}$ for $\mathrm{MM} 2$. The ellipticity enhancement of MM1 roots in the phase delay of cross-polarized component due to intra coupling. The corresponding polarization states in the far field are illustrated as an inset graph in Figure 5-4(b) where the output polarization states from MM1 and MM2 are visualized. The results highlight the potentials of the inductively coupled metasurface in functioning as a polarization rotator and a quarter wave plate with a strong optical activity and extraordinary phase delay, and provide an approach for switchable operation by combining a dynamic technique to actively control the coupling channels.

\subsection{Conclusion}

In this chapter, we have investigated the angle-dependent near-field coupling switch phenomenon in the orthogonally orientated SRR meta-molecular system. The switch 
behavior of the coupling channel is verified from the far-field co- and cross-polarized spectra and the near-field surface current distributions. We use the equivalent circuit model to describe the SRR, and find that it is the location of the equivalent circuit components that determines the near-field coupling strength in the meta-molecular system. Since the cross-polarized component induced by inductive coupling accompanies with a phase delay relative to the co-polarized radiation, we further investigate the potential applications of the near-field coupled metamaterial system in polarization control. The switchable coupling channel could also be applied in diverse areas for dynamic devices such as polarizationdivision multiplexing devices and vortex beam generation. 


\section{Chapter 6: Inter unit cell coupling effects in polarization}

\section{control}

Metamaterials are artificially designed arrays that are composed by periodically constructed unit cells with lattice period in the subwavelength scale. The intra coupled double SRR meta-molecular system is discussed in last chapter where the intra coupling channel is switchable dependent on the orientation of the SRRs. In addition to the near-field coupling between meta-atoms in the meta-molecular system, the nearest neighboring metamolecular interactions in the array also play an important role in determining the collective resonant behaviors of metamaterials. ${ }^{11,112}$ The effects originating from varied periods of lattice reflect in the coupling strength, ${ }^{112}$ diffraction mode ${ }^{111}$ and coherent resonance ${ }^{113}$ that modulate the spectral linewidth and scattering strength. The effects are especially useful for engineering the radiative losses in order to obtain high quality factor resonances. Reducing losses have always been a tough mission in optics in order to achieve high quality factor resonance for applications in lasers ${ }^{114}$ and sensors. ${ }^{102,115-117}$ Lots of attempts have been made such as using Fano resonators to excite the subradiant mode for reduced radiative $\operatorname{loss}^{101,117-121}$ and all-dielectric resonators to reduce the ohmic losses that is prominent in the metal based resonators especially in higher frequency regime. ${ }^{93,}{ }^{122}$ In addition to obtain ultra-narrow linewidth resonances, ultra-broadband resonances are also necessary for many applications in polarizing devices, ${ }^{73-75,}$ 122-125 electromagnetic absorbers, ${ }^{126,127}$ filters, ${ }^{128}$ modulators ${ }^{129,130}$ and cloaking. ${ }^{131,132}$ The strategies to enhance the bandwidth usually accompany with complex fabrication procedures and accurate 
alignment for multilayer metamaterials. ${ }^{133}$ Metasurface with single layer of structured unit cell is easy to be handled without complex fabrication flows, however, it is difficult to obtain a broadband operation. One possible approach is by the intra coupled meta-molecule discussed in last chapter where mode splitting occurs. In this chapter, we will focus on the inter coupling effects between the meta-molecules in the metamaterial array.

\subsection{Background}

(a)
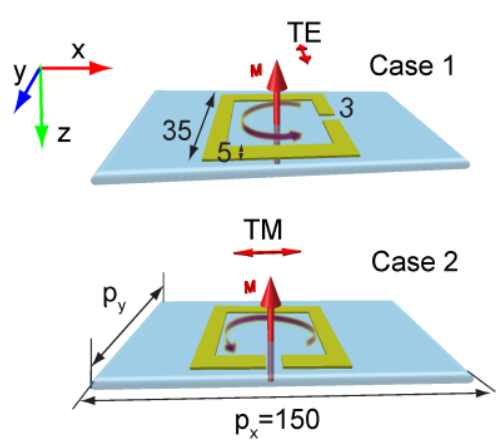

(b)

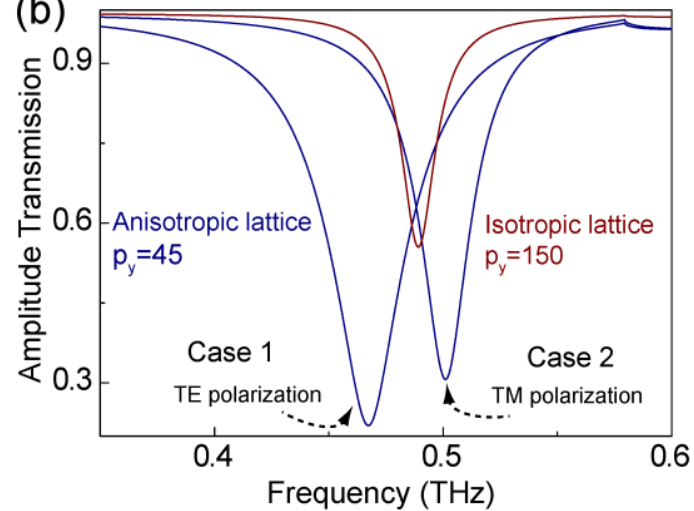

Figure 6-1. Resonator excited with LC resonance at different lattices. (a) Two cases of split ring resonator (SRR) sitting on the substrate and (b) corresponding LC resonances in transmission mode at different lattice configurations.

We still use the conventional split ring resonators (SRR) to probe the inter coupling effects whose resonance feathers have been clearly understood as shown in Figure 6-1 (a). With one split gap in the square loop, SRR possesses a bianisotropic property whose fundamental mode (LC resonance) is excited with linearly polarized light along the gap arm. ${ }^{9,} 105$ Through engineering the periodicity of the lattice where SRR resides, we are able to obtain an isotropic/anisotropic response by defining the lattice length $p_{x}$ and $p_{y}$. For simplicity, we 
keep $p_{x}$ fixed at $150 \mu \mathrm{m}$. As $p_{y}$ is not equal to $p_{x}$, the lattice is rectangular, and two cases (case 1 and case 2) are assigned as shown in Figure 6-1(a) to selectively excite the LC resonance. The corresponding spectra are exhibited by the blue lines in Figure 6-1(b) where two features are observed: 1 . eigen frequencies of the two cases deviated with each other; 2. linewidths of the resonances are different. These results are in fact predicable according to refs. 111-113 due to the different interaction strength of unit cells between the two cases. ${ }^{111-113}$ The condition will be different if $p_{y}$ is equal to $p_{x}$ which leads to a square lattice, and thus case 1 is identical to case 2 . The corresponding resonance reveals a much weaker strength since the length along $y$-axis is enlarged to $p_{y}=150 \mu \mathrm{m}$ which decreases the density of unit cells in the array, and thus lowers the scattering intensity.

In the discussion above, we keep all the parameters of the metasurface array the same except the length of lattice along $y$-axis, and investigate the nearest neighboring unit cell interaction based on the well-known LC resonance. From the features of the spectra, there are three spectral properties modulated at different lattice lengths: 1. eigen frequency shift; 2. resonant quality factor modulation; 3. resonance strength modulation. These effects are universal to periodic array with different geometric parameters from millimeter to nanometer or unit cells with more complicated constructions. In this chapter, we will focus on a more complex system and discuss the effects of inter coupling.

\subsection{Design principles}

An identical intra coupled system is adopted to investigate the effects of inter coupling 
with two orthogonally oriented SRRs in one unit cell as shown in Figure 6-2(a) that is named as a meta-molecule. As discussed in last chapter, one of the meta-atoms will be directly coupled to external field and excited the fundamental LC mode by linearly polarized incidence along the gap direction. The neighboring orthogonally oriented metaatom is induced to oscillate through the intra coupling to the directly excited meta-atom by a magnetic dipole. For simplicity of presentation, the directly excited meta-atom is called "bright" and the indirectly induced one is called "dark". The intra coupling effects on the coupling strength ${ }^{85}$ and switch ${ }^{107}$ have been discussed, and here we will only focus on the inter coupling effects. In order to implement this investigation, we design two samples as shown in Figure 6-2(c) and (d).

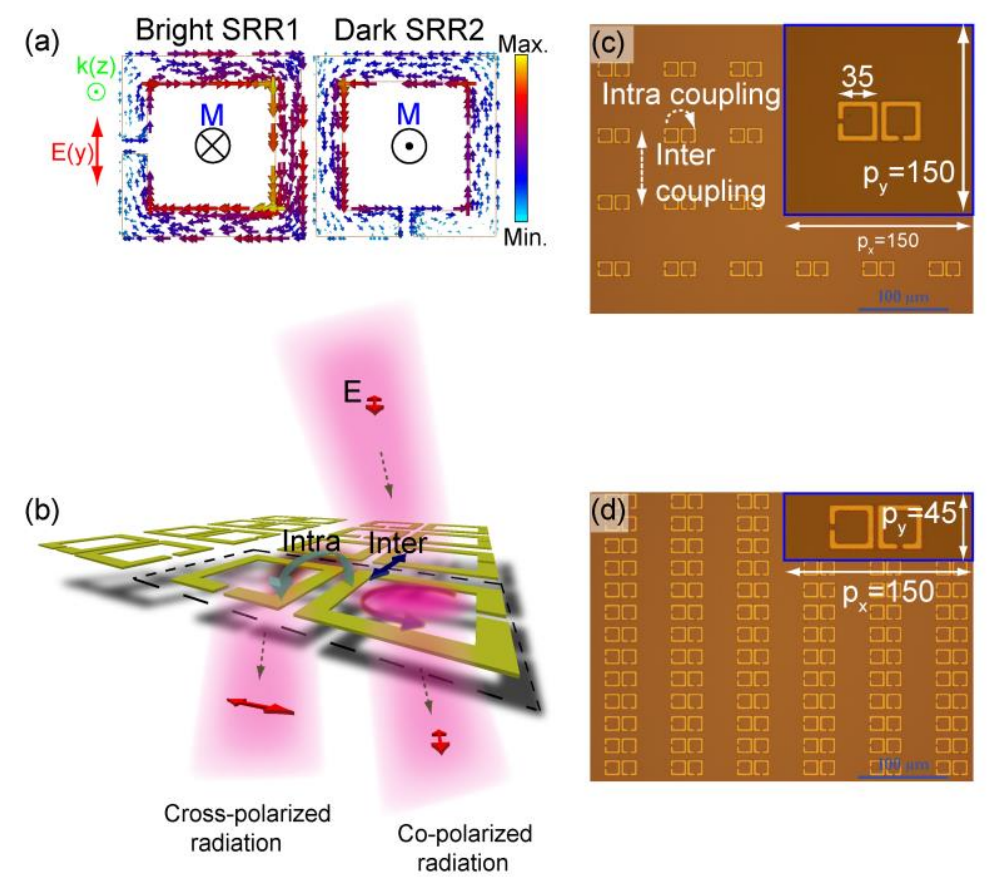

Figure 6-2. Design principle to study the inter coupling effects. (a) Design of one unit cell with two SRRs that is intra coupled through magnetic dipoles. (b) Schematic diagram of a meta-molecular array. (c) and (d) Microscopic images of two meta-molecular arrays. 
As shown in Figure 6-2(c) and (d), we keep all the parameters of the two meta-molecular unit cells identical except the distance between the meta-molecules along $y$-axis in the array that in fact engineers the periodicity of unit cell along $y$-axis. Therefore, only the coupling strength originated from the interactions between the neighboring meta-molecules is modulated. We can then study the effects by probing the far-field transmission spectra as schematically illustrated in Figure 6-2(b). Two representative cases are designed with $p_{y}=$ $45 \mu \mathrm{m}$ and $p_{y}=150 \mu \mathrm{m}$ to clearly exhibit the contrasts between them.

\subsection{Experimental data and analysis}

(a)

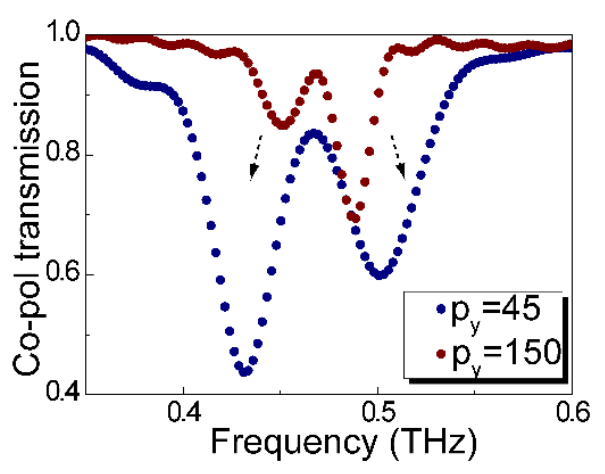

(b)

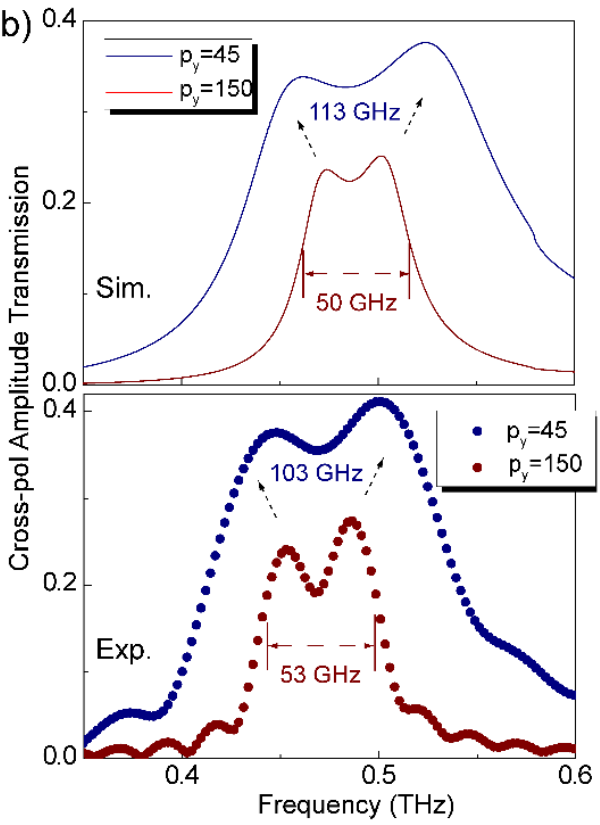

Figure 6-3. Far-field transmission spectra of the two meta-molecular arrays. (a)

Measured co-polarized transmission spectra and (b) simulated and measured crosspolarized transmission spectra.

Taking advantage of the terahertz time-domain spectroscopy system, we performed 
transmission measurements with polarizers to coherently obtain co- and cross-polarized signals in the far field, and the data are summarized in Figure 6-3. It is well known that the co-polarized spectra of the intra coupled system will reveal a mode splitting in both cases, however, by comparing the two different inter coupling scenarios, the resonance strength and mode splitting are obviously enhanced at the case of $p_{y}=45 \mu \mathrm{m}$. Meanwhile, the excitation of dark meta-atoms will always induce the radiation of cross-polarized component that is induced due to the intra coupling independent to the external excitation. Therefore, it is a direct measure of the intra coupling strength. In order to fully discover the inter coupling effects, we also probe the behaviors of cross-polarized transmission as shown in Figure 6-3(b) where both simulated and measured data are exhibited. The simulations were performed by commercially available software CST Microwave Studio using frequency domain solver through building exactly the same unit cells as the measured samples with the identical material parameters.

From the cross-polarized transmission spectra, we observe a mode splitting that matches with the co-polarized spectra. For a planar metasurface array without applying external magnetic fields, the two components of cross-polarization in the Jones matrix reveal the identical behaviors according to the reciprocity theorem, and thus we only consider the element $t_{x y}$ for simplicity. In line with the co-polarized transmission spectra, there are two effects occurring in the cross-polarized spectra by comparing the two scenarios: 1 . bandwidth broadening; 2 . amplitude enhancement; in the stronger inter coupling scenarios with $p_{y}=45 \mu \mathrm{m}$ compared to $p_{y}=150 \mu \mathrm{m}$. The bandwidth broadening originates from the 
larger eigen frequency deviation between case 1 and case 2 (see Figure 6-1(b)). The $3 \mathrm{~dB}$ bandwidth is $103 \mathrm{GHz}$ in the case of $p_{y}=45 \mu \mathrm{m}$ that is much improved compared to the scenario of $p_{y}=150 \mu \mathrm{m}$ whose bandwidth is $53 \mathrm{GHz}$. The amplitude enhancement effect attributes to the larger unit cell density at $p_{y}=45 \mu \mathrm{m}$ than that of $p_{y}=150 \mu \mathrm{m}$ (observed from Figure 6-2 (c) and (d) where the number of unit cell contributing to the scattering is different at the same visual fields). ${ }^{111}$ From experimental data, the amplitude is enhanced from around 0.26 to 0.42 in the discussed frequency range.
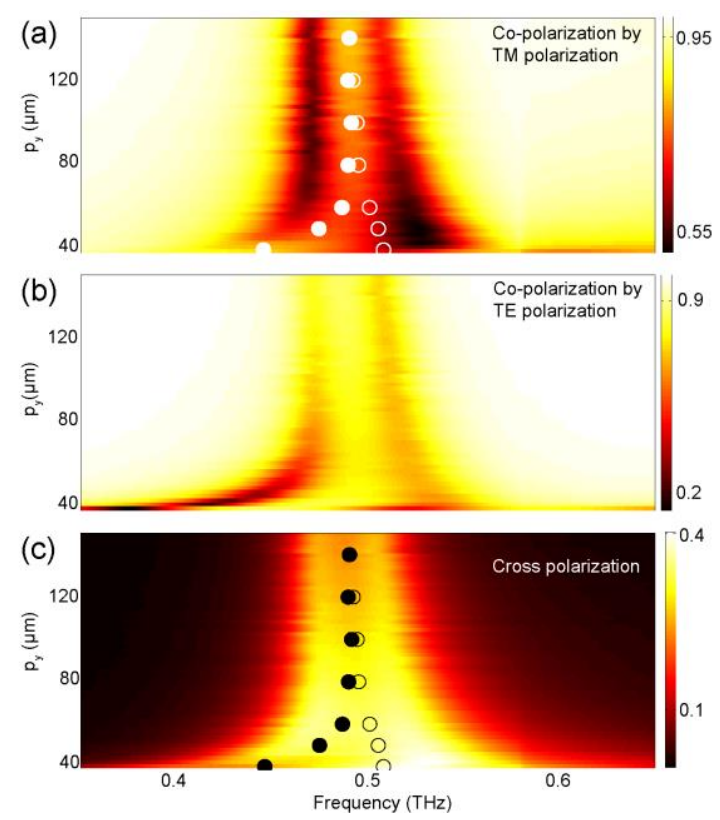

Figure 6-4. Detailed amplitude transmission spectra with varied $\boldsymbol{p}_{y}$. (a) and (b) Copolarized spectra with TM and TE polarized excitation, respectively. (c) Cross-polarized spectra. The points represent the eigen frequencies of single meta-atoms at case 1 and case 2 in the corresponding lattice parameters.

The inter coupling effects are investigated by the two scenarios discussed above, and detailed spectral evolutions by varying $p_{y}$ from 40 to $150 \mu \mathrm{m}$ in steps of $5 \mu \mathrm{m}$ are visualized 
in Figure 6-4 where both co- and cross-polarized transmission spectra are shown and compared. From all the three spectra, the effects of bandwidth broadening and amplitude enhancement is visualized by merely adjusting the lattice parameter $p_{y}$. By comparing the co-polarized spectra in Figure 6-4(a) and (b) with orthogonally polarized excitations, we observe different responses originating from the anisotropy in the rectangular lattice. The eigen frequency of the fundamental LC resonance for an individual meta-atom residing on the corresponding lattice is also plotted in the graphs with dots (for SRR1) and circles (for SRR2). The evolution trajectories of the eigen frequency exhibit a larger split with smaller value of $p_{y}$ in the rectangular lattice that is consistent with the bandwidth broadening effect in the intra coupled meta-molecular array. Therefore, we conclude that it is the modal superposition of the eigen resonances that contributes to the bandwidth broadening in the coupled system. 


\subsection{Applications in polarization control}
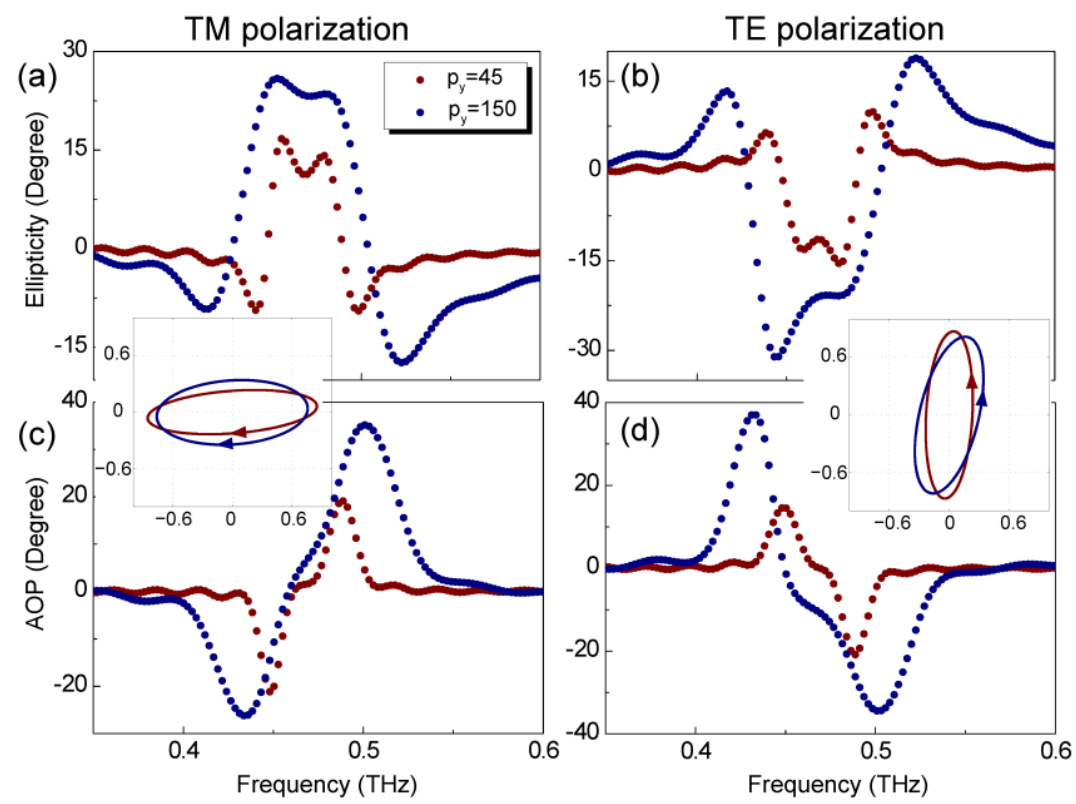

(e)

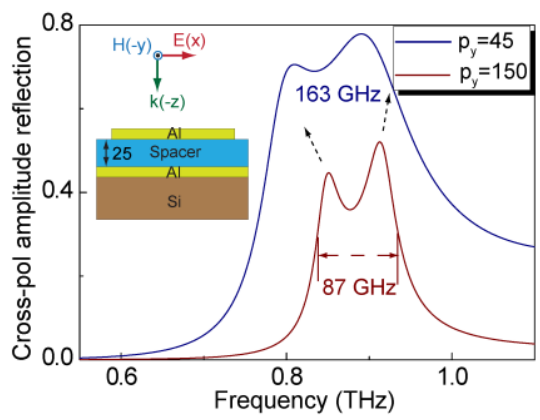

Figure 6-5. Potential applications in polarization control. (a) and (b) Calculated ellipticities of output polarization ellipse through the meta-molecular array with TM polarized and TE polarized incidence, respectively. (c) and (d) Corresponding angles of polarization ellipse for output signals. (e) Enhancement of cross-polarized signals by using the reflection mode.

Cross-polarized component in transmission mode has shown many promising potential applications for arbitrary wave front manipulation, ${ }^{7}$ flat lenses, ${ }^{19}, 20$ encoding metamaterials, ${ }^{24,25}$ cloaking $^{23}$ and holography. ${ }^{21,22,134-136}$ All these applications in devices 
require high conversion efficiency of cross-polarized component and broad operation frequency bandwidth. Therefore, an optimization of inter coupling reveals extreme significance in acquiring better device performance. For the inter coupled meta-molecular metasurface, we probe the performance optimization in terms of polarization control. The ellipticities and the angles of polarization ellipse (AOP) of output light are calculated by Stokes parameters as shown in Figure 6-5(a)-(d). Similar effects on performance enhancement are observed in the ellipticity spectra as shown in Figure 6-5(a) and (b): 1. bandwidth of the effective ellipticity spectra is broadened; 2. values of ellipticity angle are increased with stronger inter coupling strength. In addition, optical activity is also enhanced in the AOP spectra with a stronger inter coupling strength. According to the spectra, ellipticity value reaches to $-30^{\circ}$ at $0.46 \mathrm{THz}$ with $y$-polarized excitation that indicates an elliptically polarized state as insets shown in Figure 6-5. Meanwhile, AOP value reaches to $35^{\circ}$ at $0.43 \mathrm{THz}$ where its ellipticity value is about $0^{\circ}$ with $y$-polarized incidence indicating large optical activity. The output ellipticity performance from the proposed metasurface highlights the potentials to function as a broadband and high-efficiency polarizing device. The performance of AOP spectra is limited by the transmission mode where the crosspolarized amplitude is theoretically limited below 0.5 . By integrating the metasurface with a metal plate operating in reflection mode as the inset shown in Figure 6-5(e), crosspolarized component is enhanced both in amplitude and bandwidth. Such a configuration is firstly discussed as the perfect absorber device where only co-polarized component is preferred to dissipate energy. However, cross-polarized radiation channel is enhanced as an alternative channel to radiate energy by using an anisotropic unit cell. As shown in Figure 
6-5(e), the amplitude is enhanced to 0.45 , and $3 \mathrm{~dB}$ bandwidth is $87 \mathrm{GHz}$ compared to the metasurface of $p_{y}=150 \mu \mathrm{m}$ in transmission mode. Similarly, a further enhancement in reflection mode is observed in the case of $p_{y}=45 \mu \mathrm{m}$ with amplitude reaching to 0.7 and 3 $\mathrm{dB}$ bandwidth reaching to $163 \mathrm{GHz}$. By optimizing parameters of the metal-insulator-metal configuration, the meta-molecular metadevice is possible to function as a broadband and high-efficiency half wave plate.

\subsection{Conclusion}

In this chapter, we have probed the inter coupling effects in details on the spectral bandwidth and resonance strength in the meta-molecular array that is composed by orthogonally oriented intra coupled meta-atoms. The bandwidth enhancement effect attributes to the modal superposition of eigen modes in orthogonally orientated unit cells with varied lattice parameters. The amplitude enhancement is due to the stronger scattering intensity by resonators in the array with larger packing density. We also showed the performance of the inter coupled metasurface in polarization control, and proposed an approach to further enhance the bandwidth and amplitude of cross-polarized component operating in reflection mode. The arbitrary manipulation of terahertz wave by metasurface would enable diverse applications in functional devices. However, all the discussions stay in a passive mode which limits the applications. Active polarizing devices are required for the operation with dynamic functionalities. We will move to the discussion of a dynamic metadevice in next chapter. 


\section{Chapter 7. Active MEMS metasurface}

Passive metamaterials cannot meet the increasing requirements of applications anymore, especially in imaging, communication and optical encoding systems. Actively controlled metamaterials that are switched or modulated in an ultrafast speed have revealed promising potentials in cutting-edge applications. The usually adopted strategies for active modulation of the metamaterial response is by integrating dynamic materials such as silicon islands, ${ }^{58}$, ${ }^{137}$ graphene, ${ }^{92,129,130,138,139}$ liquid crystals ${ }^{140}$ and phase change materials. ${ }^{19},{ }^{141}$ However, these hybrid metadevices are only modulated globally and uniformly in the whole array by tailoring the intrinsic properties of the active media, which hinders the applications in more sophisticated functionalities such as wavefront deflection through local phase gradient, hologram and flat lenses. An alternative approach is offered by a micro-electro-mechanical system (MEMS) that enables a freedom of local control in each single resonant meta-atom. Local control in each unit cell will boost the potentials of active metadevice by an unprecedented degree. In this chapter, we will introduce a multifunctional metadevice on the basis of MEMS technique that provides a possibility to actively control the electromagnetic response by external electrostatic voltage in each unit cell. We first investigate the physical model of the proposed meta-cavity from the aspect of systemic losses. With the MEMS-enabled giant modulation of resonance properties, dynamic polarization conversion is realized by the proposed scheme though tailoring phase values. The MEMS metadevice scheme also provides possibilities to dynamically deflect the 
reflection wavefront by local control in each column of the metadevice array, and realizes real-time rewritable holographic displays by encoding the array in every single unit cell.

\subsection{Introduction}

Many fascinating phenomena ${ }^{7}, 16,23,25,38,135$ have been reported recently with the concept of metasurfaces that focused on the study of two-dimensional metamaterials and greatly release the fabrication restrictions. However, all these two-dimensional engineered metasurfaces exhibit a fixed operation function once fabricated. Recently, a phase change material (GST, germanium antimony tellurium) based metadevice employing Mie scattering was proposed and demonstrated to operate as a rewritable multifunctional device whose predesigned unit cell array is formed by a laser writing technique. ${ }^{19}$ Although the GST based rewritable metadevice was easily encoded through laser writing, such kind of approach is still unable to provide a real-time control of functionalities. An electrostatic voltage controlled MEMS resonator offers a solution for the real-time encoding of the unit cell, and thus constructs the desired local radiation in each pixel. The MEMS technique has been studied in a variety of fields ranging from microwave to terahertz and infrared regimes. Cantilevers of MEMS unit cell are controlled through numerical approaches such as thermal bimorphs, ${ }^{142}$ deformable cantilevers, ${ }^{143}$ comb drives ${ }^{144,}{ }^{145}$ and electrostatically actuated beams. ${ }^{146,147}$ A series of optical applications have been reported employing the MEMS technique in manipulation of intrinsic chirality, ${ }^{33}$ nonlinearity control, ${ }^{148}$ thermochromic metamaterials ${ }^{149}$ and programmable metasurfaces. ${ }^{150}$ 


\subsection{The basis of MEMS technique}

MEMS technique has been well developed in the recent years for applications in a deal of areas. Combining this technique with a metamaterial system would enable more sophisticated active devices. Optical response of the MEMS metadevice can be tailored by manipulating the states of cantilevers though diverse approaches as we have discussed in the introduction part. Here we employ an electrostatically regulated cantilever as the unit cell of metamaterial that is suspended due to the residual stress of the bimorph configuration which can be actuated by external electrostatic voltage. The cantilever in the MEMS system is regarded as a parallel plate capacitor whose capacitance is calculated by $C=\varepsilon \mathrm{A} / \mathrm{g}$ where $A$ is the effective area, $g$ is the distance between the plates and $\varepsilon$ represents the dielectric constant of the medium between the plates. For the MEMS system, it is the gap distance between the plates that is tailored, i.e. ' $g$ ', and thus the capacitance is given by $C=\varepsilon A /(g-z)$ where $z$ is the change of the distance. The electrostatic force $(F)$ applied by external voltage $(\mathrm{V})$ is calculated using the equation

$$
F=\frac{\partial W}{\partial z}=\frac{1}{2} \frac{\varepsilon A}{(g-z)^{2}} V^{2}
$$

where $W$ is the energy stored in the capacitor that is determined by $W=\frac{1}{2} \frac{\varepsilon A}{g-z} V^{2}$. We simply assume that a spring with constant $K$ is attached to the movable plate, and thus the equilibrium point with the applied voltage is determined by $F=K z=\frac{1}{2} \frac{\varepsilon A}{(g-z)^{2}} V^{2}$. It's noted here the gravity force is ignored since it contributes an extremely weak effect on the balance of force. By solving this equation of force balance, we can find a voltage where 
there is no solution for $z$, and at this condition, the movable plate will be pulled back touching the other plate. The voltage for this condition is the so-called pull-in voltage. Therefore, the displacement between cantilever and ground will be suddenly closed once reaching the pull-in voltage, and the cantilever will not raise back by the counter electrode as long as the electrostatic attractive force is larger than the mechanical restoring force. This pull-in phenomenon occurs at the case when the derivative of voltage to the position $z$ is zero $\left(\frac{d}{d z}\left[z(\mathrm{~g}-\mathrm{z})^{2}\right]=0\right)$ and thus the pull-in condition is calculated as $z_{p i}=g / 3$ with the pull-in voltage calculated by $V_{p i}=\sqrt{\frac{8}{27} \frac{K g^{3}}{\varepsilon A}}$. Such a pull-in voltage limits the modulation range of the cantilevers with only "ON" and "OFF" states discussed for a planar MEMS base metasurface device operating in transmission mode.

After the MEMS metadevice was fabricated by the photolithography technique, it requires a further processing to release the cantilevers whose radius of curvature $(r)$ is determined by

$\frac{1}{r}=\frac{6 n(1+\mathrm{n})\left(\mathrm{m} \sigma_{A l}-\sigma_{d}\right)}{t_{A l} E_{A l}\left[K+3 m n\left(1+\mathrm{n}^{2}\right)\right]}$,

where $K=1+4 m n+6 m n^{2}+4 m n^{3}+m^{2} n^{4}$ and $m=E_{A l} / E_{d} ; n=t_{A l} / t_{d}$. The thicknesses of metal aluminum $(\mathrm{Al})$ and dielectric aluminum oxide $\left(\mathrm{Al}_{2} \mathrm{O}_{3}\right)$ for the cantilever are denoted as $t_{A l}$ and $t_{d}$; the Young's modulus is represented as $E_{A l}$ and $E_{d}$; and the residual stresses are described by $\sigma_{A l}$ and $\sigma_{d}$, respectively. For a bimorph cantilever with length $l_{c}$, the displacement of the tip from the ground is presented by $\delta=r\left[1-\cos \left(\frac{l_{c}}{r}\right)\right]$ that 
increases with the larger $l_{c}$ and the smaller thickness $t_{A l}{ }^{151}$ In this work, we employed the metal-insulator-metal (MIM) structure which increases the distance $g$ between the effective capacitor plates by adding an insulator spacer with thickness $h$ between cantilever and bottom plate. According to the pull-in theory discussed before, the continuous modulation range of MIM system is extended from $g / 3$ to $(g+h) / 3$.

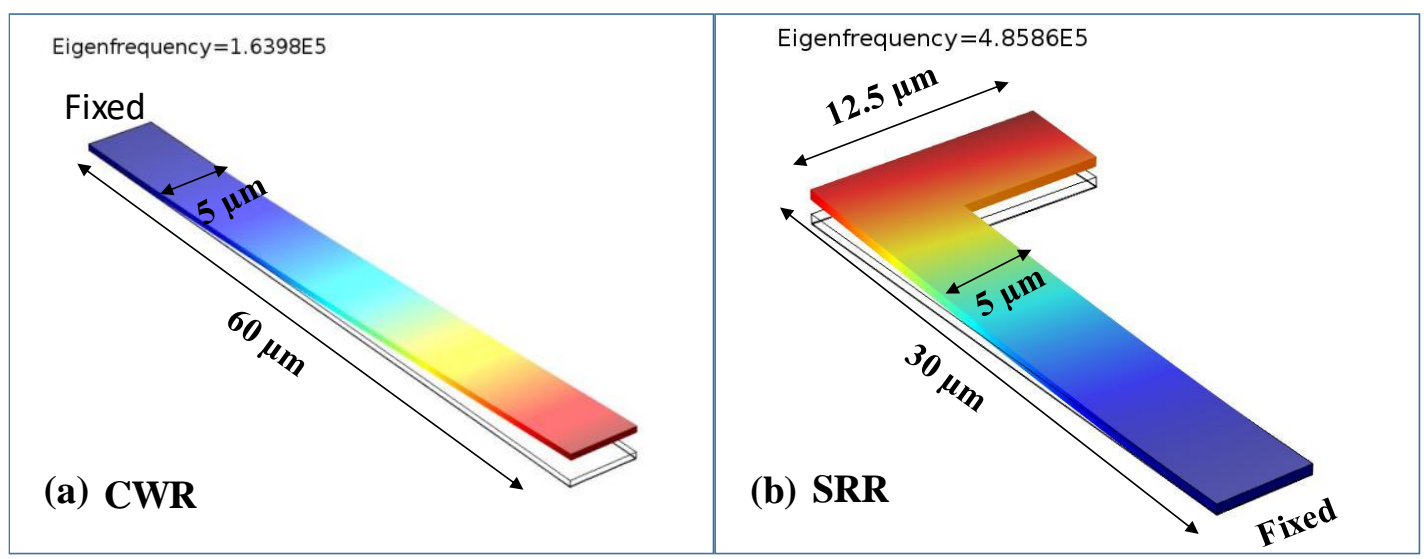

Figure 7-1. Fundamental resonance profile of the simulated deformation for (a) CWR and (b) SRR cantilevers.

The switching speed of a bimorph cantilever is one of the most important parameters to characterize the performance that is mainly limited by its intrinsic mechanical resonance frequency. We use Finite Element Modelling method to obtain the fundamental mechanical resonance frequency of the cantilevers with different configurations as shown in Figure 71. Based on the simulations, the first-order resonance frequency of cantilevers with the configuration of continuous wire resonator (CWR) is estimated to be around $164 \mathrm{kHz}$ whose geometrical parameters are shown in Figure 7-1(a). The half square ring resonator (SRR) with a shorter length as shown in Figure 7-1(b) has the fundamental resonance 
frequency around $486 \mathrm{kHz}$. Another factor of adhesion force should also be considered to estimate the switching speed when the cantilevers are pulled to touch on the ground plane at which case the strong adhesion force will adversely affect the switching speed due to the large contact area. The surface roughness, interface quality and the contact area are the factors that greatly affect the interface adhesion force.

\subsection{Disturbance theory and applications in MEMS metadevices}

For a resonance system that is slightly disturbed by an external perturbation, the shift of the resonance frequency $\left(\omega_{0}\right)$ is expressed using the disturbance theory:

$$
\frac{\Delta \omega}{\omega_{0}} \approx \frac{-\iiint d V\left[(\Delta \overleftrightarrow{\mu} \cdot \vec{H}) \cdot \vec{H}_{0}^{*}+(\Delta \overleftrightarrow{\varepsilon} \cdot \vec{E}) \cdot \vec{E}_{0}^{*}\right]}{\iiint d V\left(\mu\left|\vec{H}_{0}\right|^{2}+\varepsilon\left|\vec{E}_{0}\right|^{2}\right)}
$$

where variations of the effective permeability and permittivity tensor due to the disturbance are presented as $\Delta \vec{\mu}$ and $\Delta \overleftrightarrow{\varepsilon}$ that affect the equivalent inductance and capacitance in the equivalent circuit model of the resonator. The magnetic and electric fields are presented as $\vec{H}$ and $\vec{E}$ under the disturbance, and $\vec{H}_{0}$ and $\vec{E}_{0}$ at the original state before disturbance with the permeability $\mu$ and permittivity $\varepsilon . \vec{H}_{0}^{*}$ and $\vec{E}_{0}^{*}$ are the complex conjugates for the original fields before disturbance. The magnetic component is much weaker than the electric one while interacting with a non-magnetic medium, therefore, we only consider the electric interactions. The frequency shift $(\Delta \omega)$ is thus estimated to be approximately proportional to the part of $\Delta \overleftrightarrow{\varepsilon} \cdot \vec{E}$ for simplicity. This part $(\Delta \overleftrightarrow{\varepsilon} \cdot \vec{E})$ includes both the change of effective permittivity and the strength of electric field accumulated at the location of the resonator. Therefore, a larger modulation of the resonance by disturbance 
requires the two factors: 1 . stronger variation of the effective permittivity; 2 . stronger accumulation of the local field in the resonator.

a

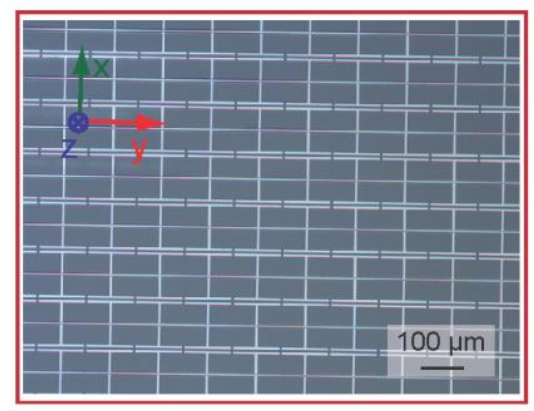

b

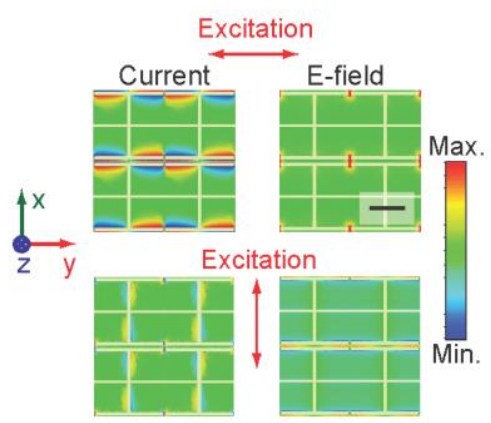

Figure 7-2. Anisotropic metadevice design. (a) Microscopic image of the sample; (b) Simulated surface current and electric field distributions.

For a simple anisotropic resonating cantilever in a MEMS system as shown in Figure 72(a), a dipolar resonance is excited with the TM polarization (linearly polarized along $y$ direction) at $0.74 \mathrm{THz}$, however, no resonance can be excited with TE polarization (linearly polarized along $x$ direction) around $0.74 \mathrm{THz}$. Corresponding resonance properties are exhibited in Figure 7-2(b) where the surface current and electric field distributions are calculated using the CST Microwave Studio. From the figures, we further confirm that there will be strong field accumulations with $y$-polarized excitation but extreme weak field accumulations with $x$-polarized excitation. For a MEMS metadevice, the intrinsic effective permittivity will be obviously adjusted by tailoring the cantilevers, and thus there will be a strong modulation of the dipolar resonance with $y$-polarized excitation. However, no modulation is expected at the case of $x$-polarized excitation according to the disturbance theory due to the weak field accumulation. Therefore, negligible influence of the spectral 
features with $x$-polarized excitation can be observed where the local electric field accumulation is weak as shown in Figure 7-2(b). In this way, the anisotropy of the MEMS metadevice is engineered through tailoring the cantilevers.

\subsection{Active switch of losses in a metadevice system}

Using the MIM metadevice, a large dynamic modulation range is achievable by enhancing the field accumulation that operates in the reflection mode. Such a MIM system was initially investigated for a perfect absorber and developed a series of applications. ${ }^{3,5} \mathrm{~A}$ typical MIM metadevice consists of three layers: a structured metasurface, a spacer made of insulator and a continuous metallic plane sitting on the substrate as shown in Figure 73(a). No transmission signal is allowed due to the existence of the ground metallic plane, and only reflective signal is considered. Therefore, a one-port single-mode resonator model can be employed to describe the dipole mode resonant properties for the spectrally separated resonances based on the coupled-mode theory (CMT). ${ }^{139,} 152$ The reflection coefficient ( $\tilde{r})$ using the CMT model is presented below:

$\tilde{r}=-1+\frac{2 / \tau_{r}}{-i\left(\omega-\omega_{0}\right)+1 / \tau_{a}+1 / \tau_{r}}$

where $\tau_{a}$ and $\tau_{r}$ represent the lifetimes of oscillation inside the resonator due to the intrinsic absorptive and radiative losses, respectively, and the resonance frequency is $\omega_{0}$. Quality factor $(Q)$ is related to the intrinsic losses of the system defined as $Q=\omega_{0} \tau / 2$ and so the reflection coefficient can also be presented by quality factors:

$\tilde{r}=-1+\frac{1 / Q_{r}}{-i\left(\omega / \omega_{0}-1\right)+1 / 2 Q_{a}+1 / 2 Q_{r}}$ 
which clearly states that it is the intrinsic absorptive $\left(Q_{a}\right)$ and radiative $\left(Q_{r}\right)$ quality factors that determine the spectral features of the one-port single-mode model. The perfect absorption occurs at the condition of $Q_{a}=Q_{r}$ based on the equation 7-5, and the inequality between the two quality factors will give rise to a decrease of the absorbance. However, we are unable to distinguish the cases of $Q_{a}>Q_{r}$ (underdamped region) and $Q_{a}<Q_{r}$ (overdamped region) merely from the amplitude spectra. As observed from Figure 7-3(b) where the reflection amplitude spectra are calculated based on the CMT, they exhibit the identical spectral features in reflection amplitude when the absolute values of $\triangle Q$ $\left(\Delta Q=\left(Q_{a}-Q_{r}\right) /\left(Q_{a}+Q_{r}\right) \times 100 \%\right)$ are equal at the two scenarios. Therefore, only the information of amplitude spectra is not enough to describe the balance between the two quality factors, and phase response $(\varphi)$ should also be taken into account which conveys the information of the imaginary part of equation 7-5. From the phase spectra as shown in Figure 7-3(c) calculated from CMT, the difference is obviously visualized between the underdamped state and overdamped state where the phase span sweeps a full $360^{\circ}$ range across the center of resonance in the underdamped state $\left(Q_{a}>Q_{r}\right)$, and the phase span sweeps less than $180^{\circ}$ range as the frequency $(\omega)$ goes across the center resonance frequency $\left(\omega_{0}\right)$.
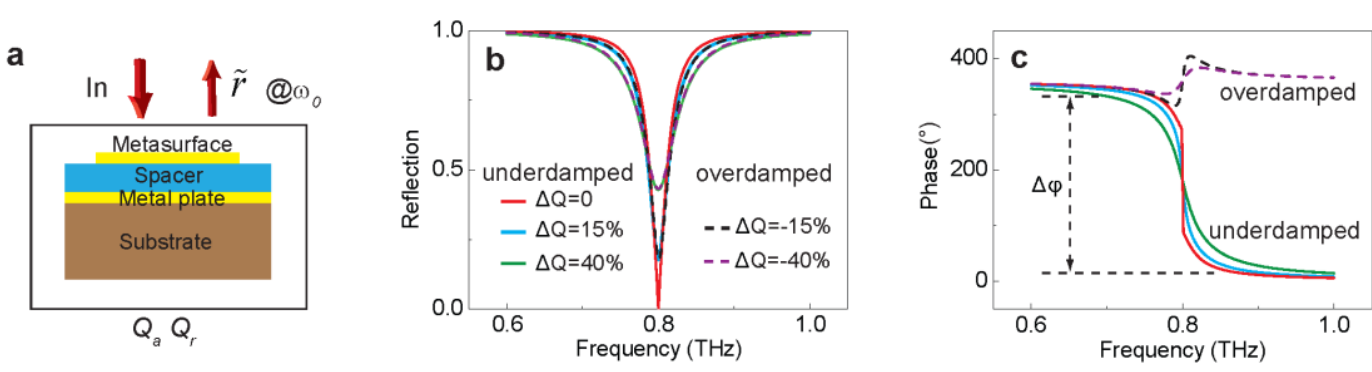
Figure 7-3. A metal-insulator-metal (MIM) system described using the coupled-mode

theory (CMT). (a) Schematic illustration of the MIM metadevice described by quality factors $\left(Q_{a}\right.$ and $\left.Q_{r}\right)$ at center resonance frequency $\left(\omega_{0}\right)$ with incident (In) and reflective $(\tilde{r})$ signals. (b) Reflection amplitude spectra and (c) phase spectra calculated by CMT in the underdamped and overdamped regions. Here $\Delta Q=\left(Q_{a}-Q_{r}\right) /\left(Q_{a}+Q_{r}\right) \times 100 \%$.

A variety of applications could be enabled through tailoring the balance between the two quality factors that in fact modulates the fundamental loss channels of the system. A more advanced metadevice can be designed to engineer the loss balance using MEMS technique that actively tunes the two loss channels according to the mode-expansion theory (MET). ${ }^{153}$ Usually, the MET is quantitatively valid for resonators that possess the high quality factor resonances, and it has been demonstrated to be effective in the MIM resonator. Considering a simple subwavelength grating unit cell with period $d$, slot width $a$, metal thickness $h_{m}$ and spacer thickness $h$, the radiative quality factor is derived as:

$Q_{r}=\frac{1}{2 k_{0} h} \sum_{m} \Gamma(m) \frac{\sin ^{2}(m \pi a / d)}{(m \pi a / d)^{2}}$

where $\Gamma(m)=\varepsilon k_{0}^{2}\left[(2 m \pi / d)^{2}+\varepsilon k_{0}^{2}\right] /\left[(2 m \pi / d)^{2}-\varepsilon k_{0}^{2}\right]^{2}$ weights the $m$ th mode contribution in the MIM cavity and $\varepsilon$ is the spacer permittivity. Here, we ignore the ohmic losses from metal and spacer layer since they will be considered into the absorptive quality factor. From the definition of quality factor, the absorptive quality factor is derived as:

$Q_{a}=\frac{\omega_{0} U}{P_{d}+P_{m}}=\frac{\operatorname{Re}(\varepsilon) h d}{\alpha \operatorname{Im}(\varepsilon) h d+\beta(2 d-a) H(\delta)}$

where $U$ is the total stored energy inside the MIM cavity, and $P_{d}$ and $P_{m}$ are the absorbed 
energy by spacer and metal, respectively. $\alpha$ and $\beta$ describe the relative contribution in absorption by spacer and metal, respectively, and $H(\delta)=\delta\left(1-e^{-2 h_{m} / \delta}\right)$ with $\delta$ being the skin depth $\left(\delta=\sqrt{2 / \mu_{0} \omega_{0} \sigma_{m}}\right)$.

From the two equations 7-6 and 7-7, we are able to clearly understand the parameters that tune the system losses. In addtion to the geometrical and material parameters of the resonator, the spacer thickness $(h)$ plays a dominant role in these two equations where $Q_{r}$ scales inverely to $h$ and $Q_{a}$ is almost linearly dependent on $h$. Therefore, the transition from overdamped to underdamped state is easily accessed by tailoring $h{ }^{138}$
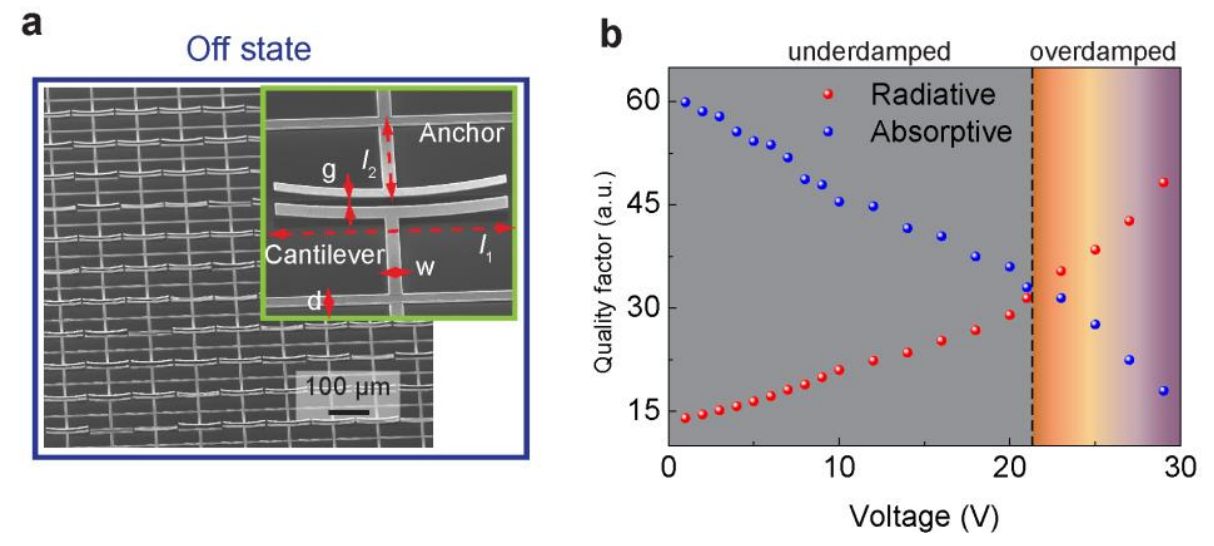

Figure 7-4. Active switch of operation state by a MEMS metadevice. (a) Microscopic image of the MEMS samples in "OFF" state (released state) and inset is the SEM image of one unit cell. Geometrical parameters of the unit cell are $g=4, w=6, d=4, l_{1}=100$ and $l_{2}$ $=50($ in $\mu \mathrm{m})$. Thickness of spacer $\left(\mathrm{SiO}_{2}\right)$ is $t=5 \mu \mathrm{m}$, structured unit cell is made of aluminum with thickness of $500 \mathrm{~nm}$, and the plate reflector is $1 \mu \mathrm{m}$ thick. Period of the unit cell is $104 \mu \mathrm{m}$. (b) Evolution of radiative and absorptive quality factors with varied voltages applied on the MEMS metadevice. 
We fabricated the anisotropic MEMS samples as shown in Figure 7-4(a) with the cantilevers at the initially released state that is defined as "OFF" state without applying external voltage. The detailed geometrical parameters are indicated in the SEM images. When the metadevice is applied with an external voltage between the metasurface and the ground plane, the suspended cantilevers are pulled close to the spacer layer by the attractive force due to the voltage, and the suspending height of the cantilever is tailored dependent on the level of the voltage. The cantilevers are pulled down to the ground at the pull-in voltage of $30 \mathrm{~V}$ that is defined as the "ON" state as shown in Figure 7-2(a). In order to investigate the modulation of quality factors by actively changing the applied voltage, we retrieved the radiative as well as absorptive quality factors of the metadevice at varied voltages as shown in Figure 7-4(b). From the graph, it is clearly presented that the metadevice operates in the underdamped region at "OFF" state where $Q_{a}>Q_{r}$. Once the external voltage is applied, the difference between radiative and absorptive quality factors is diminishing where the absorptive quality factor becomes smaller and the radiative quality factor increases at the larger voltage. The equality occurs at the voltage of $\sim 21 \mathrm{~V}$ and the operation state is then switched to overdamped region $\left(Q_{a}<Q_{r}\right)$ when the voltage is larger than $21 \mathrm{~V}$ as indicated in the Figure 7-4(b). The modulation of the two quality factors can be interpreted from the MET discussed above. In this MEMS medadevice system, the effective spacer thickness is modulated while the height of suspended cantilevers is actively tailored. 
a

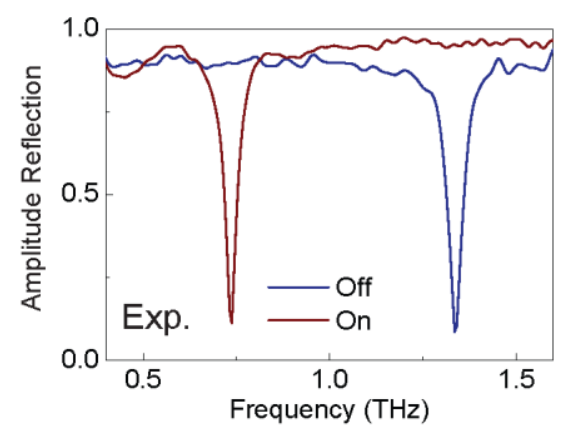

b

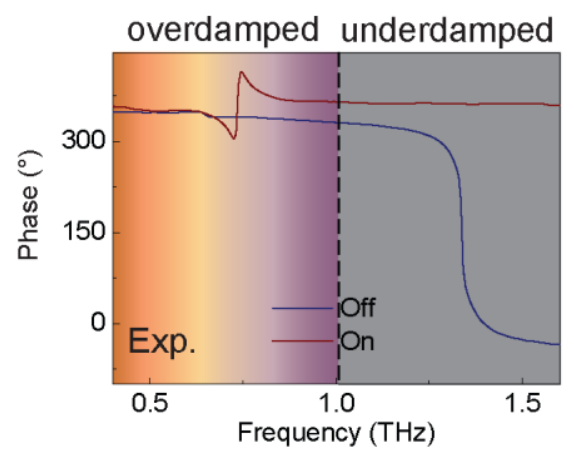

C

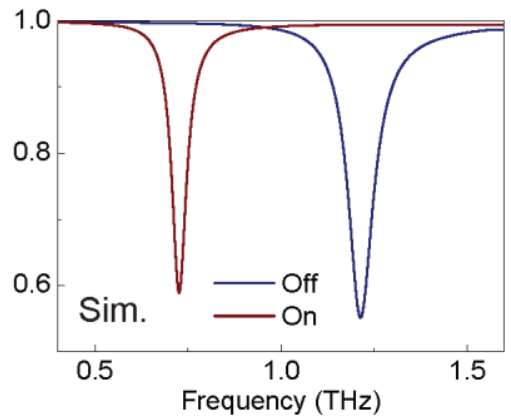

d

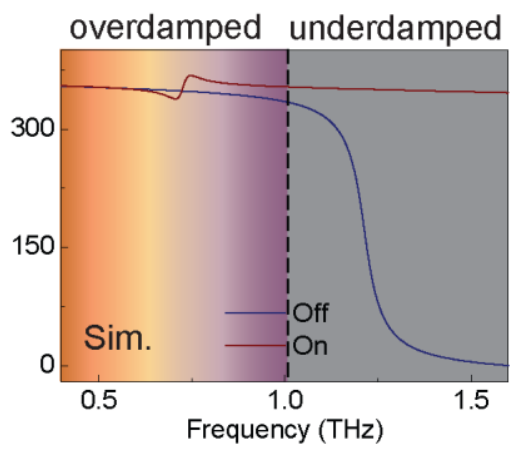

Figure 7-5. Experimental and simulated results of active switching in operation state.

(a) Experimental results of reflection amplitude and (b) phase spectra for the metadevice at "ON" and "OFF" states. The corresponding simulated results of (c) reflection amplitude and (d) phase spectra.

Terahertz time-domain spectroscopy system (THz-TDS, TeraView TPS Spectra 3000) was used to experimentally measure the reflection response, and the amplitude and phase response are extracted using Fourier transform that are normalized to the reference signal measured in dry air. The measured results are shown in Figure 7-5(a) and 7-5(b) where the reflection amplitude and phase spectra are exhibited while the metadevice operating at "ON" and "OFF" states, respectively. With the $y$-polarized incidence, the metadevice at "OFF" state reveals a strong resonance with central frequency at $1.34 \mathrm{THz}$, and the resonance is 
red shifted to $0.74 \mathrm{THz}$ when the metadevice operates at "ON" state with the external voltage of $30 \mathrm{~V}$. From the amplitude spectra alone, we are not able to judge which state the metadevice is operating at. From the phase spectra as shown in Figure 7-5(b), the contrast is obvious by observing the phase $\operatorname{span}(\Delta \varphi)$ across the resonance frequency where the phase span reveals the full $360^{\circ}$ sweep when the metadevice is at "OFF" state, and it is less than $180^{\circ}$ when the metadevice operates at "ON" state. The phase spectra clearly state that the operation state of the metadevice is switched from underdamped region to overdamped region. Such a dynamic switch of operation state is experimentally demonstrated by the MEMS metadevice. The switching speed is estimated in the $\mathrm{kHz}$ level, which is limited by the first order mechanical resonance frequency of the cantilevers, and the adhesion force of the interface between cantilevers and spacer surface.

This switching behavior was also investigated through simulations by CST Microwave Studio whose data are exhibited in Figure 7-5(c) and (d), where the reflection amplitude and phase spectra match well to the experimental data. Together with the switch of operation state, the resonance frequency of the metadevice is largely modulated by $0.6 \mathrm{THz}$ ( $\sim 45 \%$ of the resonance frequency at "OFF" state) that originates from the dipolar resonance. The equivalent capacitance and inductance of the MEMS system are tailored when the configuration of the suspended cantilevers is modulated.

Using the carefully designed MEMS metadevice, quality factors of the system are tailored in order to switch the operation state from underdamped ("OFF" state) to overdamped state 
("ON" state). A deal of promising applications could be enabled by this active MEMS metadevice. For example, the value of phase span across the resonance frequency is extremely sensitive to the external disturbance when the metadevice operates in the overdamped region, which could be employed for ultrasensitive sensors. The constant $360^{\circ}$ phase span in the underdamped region would be promising for applications in the phase encoded devices such as wavefront deflection by planar phase gradient, ${ }^{7}$ hologram, ${ }^{22}$ flat lenses, ${ }^{19}$ polarization control ${ }^{125}$ and encoded metamaterials. ${ }^{24,}{ }^{25}$ Next sections, we will focus on the underdamped operation of the MEMS metadevice, and present its capacity to operate as an active polarization convertor, a dynamic reflective wavefront deflector and a real-time rewritable holographic display device.

\subsection{Active polarization control}

We have demonstrated several approaches for efficient polarization control in the preceding chapters, however, most of them operate in a passive way. Here we will introduce an approach of active polarization control using the anisotropic MEMS metadevice whose modulation speed could reach the $\mathrm{kHz}$ level. The anisotropy of the MEMS metadevice is engineered by regulating the cantilevers that enables the modulation of phase difference between the two orthogonal polarization states. Such an active polarization control metadevice would find applications in communication systems as a multiplexer for polarization-division multiplexing in order to improve the information carrier capacity. 
The amplitude attenuation and phase response of the scattered light along orthogonal axes are varied due to the anisotropy that is free to be tailored by the artificially designed metamaterial unit cell. The reflection from the metamaterials with the linearly polarized incidence of a certain angle is mathematically presented by the equation:

$$
\hat{J}\left[\begin{array}{l}
E_{x} \\
E_{y}
\end{array}\right]=\left[\begin{array}{cc}
r_{x y} e^{i \delta} & 0 \\
0 & 1
\end{array}\right]\left[\begin{array}{c}
\cos \theta \cdot e^{-i \delta^{\prime}} \\
\sin \theta
\end{array}\right]=\left[\begin{array}{c}
r_{x y} e^{-i\left(\delta^{\prime}-\delta\right)} \cos \theta \\
\sin \theta
\end{array}\right]
$$

Here, $\hat{J}$ is the Jones Matrix of the metadevice, $\theta$ and $\delta$ are the angle of the incident polarization and the phase difference between the orthogonal components, and $r_{x y}$ and $\delta$ represent the anisotropy of the metadevice where $r_{x y}$ is the amplitude attenuation ratio $\left(r_{x y}=\left|\tilde{t}_{x}\right| /\left|\tilde{t}_{y}\right|\right)$ and $\delta\left(\delta=\varphi_{y}-\varphi_{x}\right)$ is the phase difference induced by the metadevice. $r_{x y}$ and $\delta$ originate from the anisotropy of the metadevice that has been demonstrated tunable with external voltage using the perturbation theory, and thus the output polarization state can be modulated. The linearly polarized (LP) incidence is the most commonly used state whose phase difference $\delta^{\prime}$ is $0^{\circ}$ or $180^{\circ}$, and the azimuth $\theta$ is easily rotated to meet the desired requirements of the output polarization states. In this way, we can obtain any polarization state if the phase difference induced by the metadevice is tuned covering the $360^{\circ}$ range. 
a

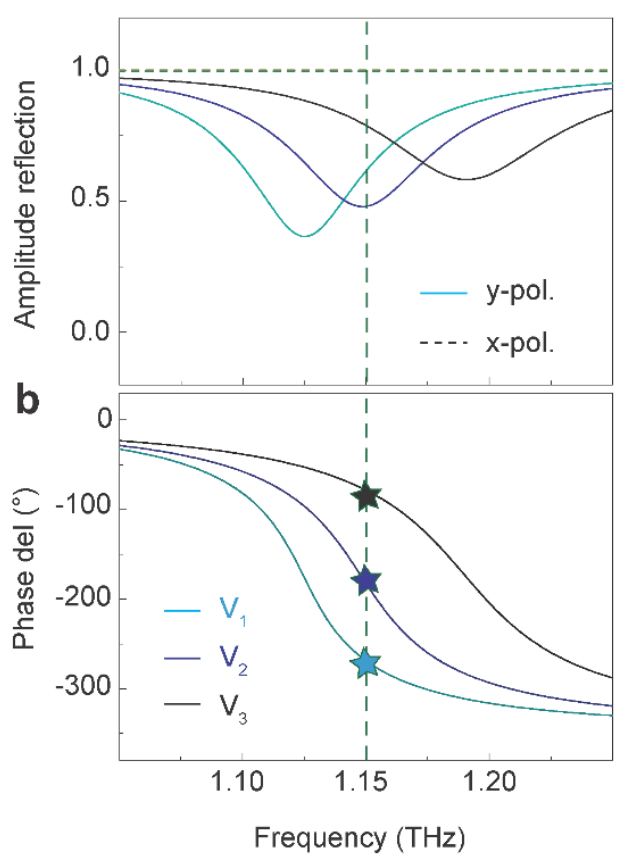

C
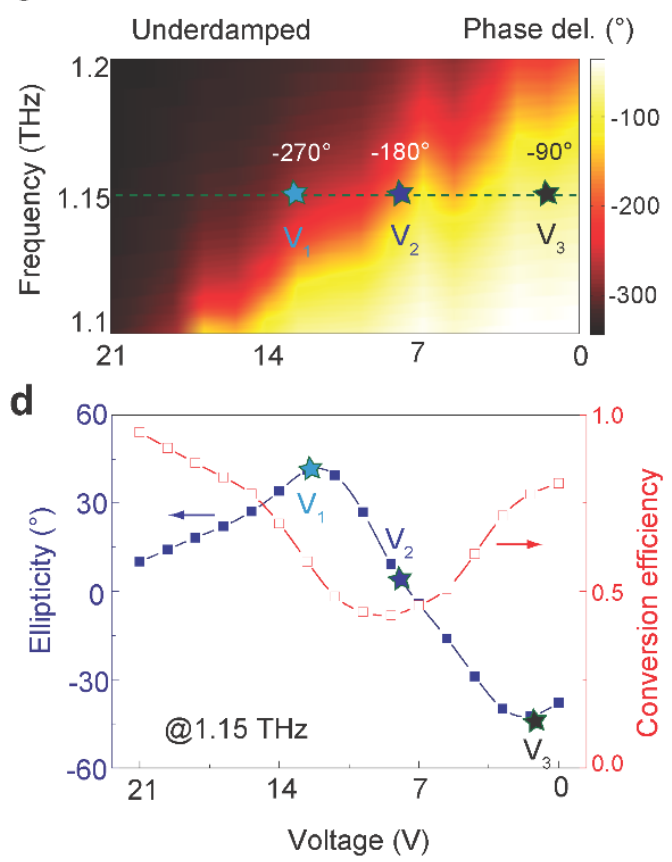

- Incident pol.

e

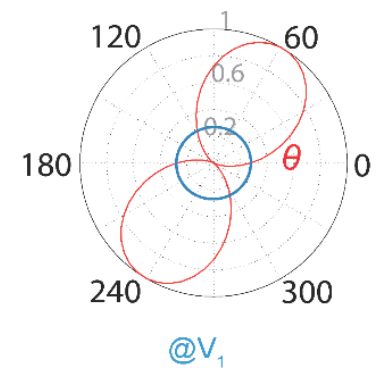

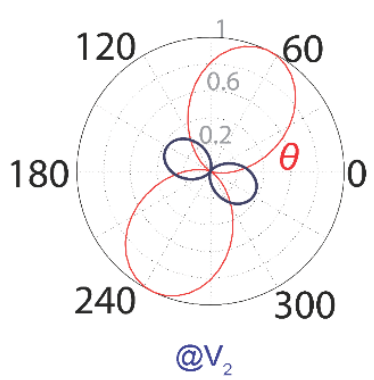

@ $\mathrm{V}_{2}$

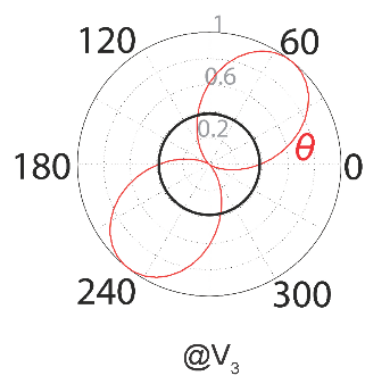

Figure 7-6. Active polarization control performance of the MEMS metadevice. (a)

Reflection amplitude spectra of the MEMS metadevice at three voltages with $y$-polarized (solid lines) and $x$-polarized (dash lines) incidence. (b) The corresponding three phase difference spectra at different voltages. (c) Continuously tuned phase difference spectra at varied voltages. (d) Polarization conversion performance on the ellipticity of the output light and the conversion efficiency of the metadevice at $1.15 \mathrm{THz}$. (e) Schematic illustrations of the output polarization states at three voltages. 
We have demonstrated that the MEMS metadevice provides a $360^{\circ}$ phase span across the resonance frequency while operating in the underdamped region, and the resonance frequency is actively tuned by regulating the cantilevers. The modulated resonance frequency provides a possibility to engineer the phase difference. We note that it is the phase difference that we need to consider for the polarization control applications in this section. Next, I will show how the phase difference is tailored by the MEMS metadevice. The proposed anisotropic metadevice gives rise to a dipolar resonance with $y$-polarized incidence whose reflection amplitude spectra are shown in Figure 7-6(a) by the solid lines, however, no resonance is excited by $x$-polarized as revealed by the dash lines in this graph where the incidence is totally reflected. The tuning of anisotropy is realized by the shift of resonance frequency by tailoring the angle of the suspended cantilevers in the MEMS metadevice through applying varied voltages as shown in Figure 7-6(a). The accompanied phase spectra for $y$-polarized excitation is also shift. As we have discussed, no resonance is excited with $x$-polarized excitation, and thus no modulation is observed for both amplitude and phase spectra according to the perturbation theory. Therefore, the phase difference along the orthogonal components at a fixed frequency is tailored through tuning the angle of the cantilevers by applying the corresponding voltages as shown in Figure 7-6(b). If the frequency tuning range in the underdamped region is large enough, any value of phase difference ranging from $0^{\circ}$ to $360^{\circ}$ will be accessible. In our case, the operation state is switched to overdamped region at $21 \mathrm{~V}$, and thus the modulation within underdamped region from 0 to $21 \mathrm{~V}$ provides a tuning of phase difference ranging from $-75^{\circ}$ to $-340^{\circ}$ as shown in Figure 7-6(c). Three typical phase difference values $\left(-90^{\circ},-180^{\circ}\right.$ and $\left.-270^{\circ}\right)$ at 
$1.15 \mathrm{THz}$ are obtained when the voltage is given at $\mathrm{V}_{1}=13 \mathrm{~V}, \mathrm{~V}_{2}=8 \mathrm{~V}$, and $\mathrm{V}_{3}=1 \mathrm{~V}$, respectively, that enable the circular and linear output polarization states. From Figure 7-6 (d), the calculated ellipticity spectra of the output light together with the conversion efficiency are presented, where we observe an efficient and continuous modulation of polarization states. Right-handed circular polarization (RCP), linear polarization (LP) and left-handed polarization (LCP) are obtained at $\mathrm{V}_{1} \mathrm{~V}_{2}$ and $\mathrm{V}_{3}$, respectively. More polarization states are also achievable through accurately applying the voltage on the MEMS metadevice.

In order to function as a quarter wave plate (QWP) or half wave plate (HWP), we would require the angle $\theta$ of the incident linear polarization to satisfy $r_{x y} \cos \theta=\sin \theta$ and thus $\theta=\arctan \left(r_{x y}\right)$. From Figure 7-6(e), we can observe the polarization states of incident and output light at the three typical voltages where the incident is linearly polarized with angle $\theta$ and the output lights are RCP, LP and LCP, respectively. Thus far, we have demonstrated the active polarization conversion using the MEMS metadevice by universal control of the entire resonator array. Many promising applications can be developed using the MEMS metadevice scheme, such as polarization related communication, display and encoding fields. In addition to the universal control of the entire array, the electrostatic cantilevers can also be separately controlled in each single column or in every single unit cell that can be described as a one-dimensional (1D) and two-dimensional (2D) control. Next two sections, we will discuss the potential applications with 1D and 2D control techniques in dynamic wavefront deflection and real-time rewritable holographic display. 


\subsection{Dynamic wavefront deflection}

The generalized Snell's law was proposed by Yu et al in 2011 and demonstrated with crosspolarized component using the "V" shape resonators in transmission mode. ${ }^{7}{ }^{16}$ However, the efficiency of cross-polarized component is limited for the planar metasurfaces whose theoretical maximum is $25 \%$ for the energy conversion ${ }^{154}$, and once the metasurface is patterned, the functionality is no longer able to be reconfigured. Therefore, a more efficient and flexible approach is required for the dynamic applications in metasurface based planar optics. The reflective mode provides a higher operation efficiency ${ }^{22}$ and the MEMS technique provides a pathway of dynamic control. Conventional methods for the active control of optical response integrating hybrid metamaterials only provide a universal control of the entire array collectively through integrating with graphene monolayer ${ }^{129,} 130$, ${ }^{155}$ or ion planted silicon islands, ${ }^{58,137}$ which are not able to provide a local control in each column or each single unit cell. For the electrostatic cantilever MEMS metadevice, it provides the freedom of local control by separately applying the encoded voltages to the selected column (1D) or unit cells (2D). Here in this section, I will discuss one possible scheme of a MEMS metadevice to selectively control the different columns and demonstrate the dynamic wavefront deflection by applying different encoding sequences in reflection mode. 
a
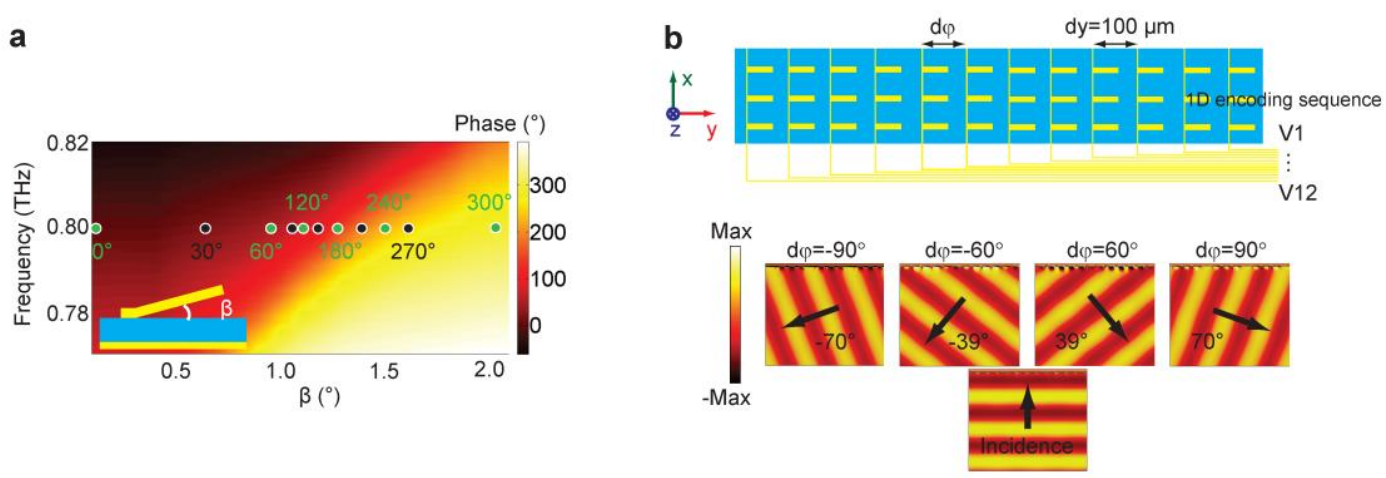

Figure 7-7. Proposed scheme of the dynamic reflection wavefront deflection. (a) Phase response of the single wire MEMS metadevice at varied cantilever angles $(\beta)$ at $0.8 \mathrm{THz}$. (b) One possible scheme of dynamic reflection wave deflector by selective control of 12 columns. The reflective wavefront is directed to $\pm 39^{\circ}$ by repeating 6 -digit encoding $(\mathrm{d} \varphi=$ $\left.\pm 60^{\circ}\right)$, and it is directed to $\pm 70^{\circ}$ by repeating 4-digit encoding $\left(\mathrm{d} \varphi= \pm 90^{\circ}\right)$ with normal incidence.

The generalized Snell's law provides the freedom to artificially engineer the direction of wavefront by controlling local phase gradient of the surface whose deflection angle is determined by:

$\theta_{r}=\arcsin \left(\frac{\lambda_{0}}{2 \pi} \frac{d \varphi}{d y}\right)$

Here the reflective wavefront direction angle is $\theta_{r}$, vacuum wavelength of light is $\lambda_{0}$ and the $1 \mathrm{D}$ phase gradient along $y$ axis is described by $\frac{d \varphi}{d y}$. By tailoring the phase gradient item, the direction of wavefront is modulated. For a MEMS metadevice, the period of the unit cell is fixed which provides a constant $d y$. Therefore, the deflection angle is controlled by modulating the phase shift $(d \varphi)$ item between the neighboring unit cells. With this 
understanding, we can dynamically modulate the angle of wavefront through encoding the metadevice array in the column level (1D) along $y$ axis with a varied encoding voltage sequence. For the repetitive encoding, the phase span of $360^{\circ}$ is required and thus the metadevice is still required to operate in the underdamped region. As a proof of concept, we redesign the unit cell using a simple wire as the cantilever to excite the dipolar resonance, and it operates in the underdamped region within the entire modulation range. The longer length of cantilevers will provide a larger initial height of the suspended cantilevers so that it gives rise to a larger frequency shift from the initial state to the pull-in state. The suspended resonator is an aluminum strip whose length is $60 \mu \mathrm{m}$, width is $15 \mu \mathrm{m}$ and thickness is $500 \mathrm{~nm}$, and the spacer $\mathrm{SiO}_{2}$ is enlarged to $10 \mu \mathrm{m}$ as the inset graph illustrated in Figure 7-7(a) and (b). The anchor is also made of aluminum whose width is $4 \mu \mathrm{m}$ and thickness is $500 \mathrm{~nm}$. A dipole resonance is excited as the fundamental mode with $y$ polarized incidence whose frequency is modulated by tailoring the angle $\beta$ of cantilevers relative to the spacer due to the electrostatic force. With this metadevice, the radiation phase is continuously modulated covering larger than $330^{\circ}$ at $0.8 \mathrm{THz}$ when the voltage is applied from 0 to $30 \mathrm{~V}$ (pull-in voltage) as shown in Figure 7-7 (a).

With the continuously modulated radiation phase response as shown in Figure 7-7(a), we can selectively choose 6-digit or 4-digit encoding of the column in the array as shown in Figure 7-7(b). For the 6-digit encoding, the encoding sequence of the phase response is $0^{\circ}$ $\left(\mathrm{V}_{1}\right), 60^{\circ}\left(\mathrm{V}_{2}\right), 120^{\circ}\left(\mathrm{V}_{3}\right), 180^{\circ}\left(\mathrm{V}_{4}\right), 240^{\circ}\left(\mathrm{V}_{5}\right)$, and $300^{\circ}\left(\mathrm{V}_{6}\right)$ for the six consecutive columns that is repeated in the entire array. In this way, the phase shift is $d \varphi=60^{\circ}$ and the 
calculated reflective wavefront deflection angle is $39^{\circ}$ that is demonstrated by the simulated electric field distributions as shown in Figure 7-7(c). Simulated reflective wavefront is in good quality with plane wave incidence, and the direction of reflection wave is towards around $39^{\circ}$ relative to the incidence. The encoding sequence is easily changed by the external voltage. If the 6-digit sequence is inversed as $300^{\circ}\left(\mathrm{V}_{1}\right), 240^{\circ}\left(\mathrm{V}_{2}\right), 180^{\circ}\left(\mathrm{V}_{3}\right), 120^{\circ}$ $\left(\mathrm{V}_{4}\right), 60^{\circ}\left(\mathrm{V}_{5}\right)$, and $0^{\circ}\left(\mathrm{V}_{6}\right)$, the wavefront direction is dynamically switched towards $-39^{\circ}$ relative to the incidence. This is verified in the simulated electric distributions in Figure 77(c). Similarly, we can also change the encoding sequence to 4-digit which will provide a larger phase shift with sequence of $0^{\circ}\left(\mathrm{V}_{1}\right), 90^{\circ}\left(\mathrm{V}_{2}\right), 180^{\circ}\left(\mathrm{V}_{3}\right)$ and $270^{\circ}\left(\mathrm{V}_{4}\right)$. For the 4digit encoding with $\mathrm{d} \varphi=90^{\circ}$, the theoretical wavefront direction is towards $70^{\circ}$ according to the general Snell's law that is well reproduced by the simulated electric field distribution. $-70^{\circ}$ direction is also able to be dynamically realized by inversing the encoding sequence.

It is noted that we ignore the amplitude modulation accompanied with the encoded phases which is randomly distributed along the 1D surface. The amplitude can also be encoded using a more sophisticated design, which will further improve the quality of the wavefront. ${ }^{156,157}$ So far, we have demonstrated the dynamic deflection of the reflective wavefront using the MEMS metadevice through 1D control in the column of the resonator array. Next section, the local control of each unit cell in the entire array of the metadevice will be discussed for application in real-time rewritable holographic display. 


\subsection{Real-time rewritable holographic display}

Nowadays, local control in the unit cell level is also extensively discussed that have found many applications such as hologram. ${ }^{24,135,136,158}$ For traditional hologram, the commonly employed method is by etching different depths on a transparent medium for phase-only computer generated hologram $(\mathrm{CGH})$, however, there are several issues limiting the applications such as the twin images and the low diffraction efficiency. ${ }^{22}$ The efficiency can be improved by the recently developed geometric metasurface where each unit cell offers the required local radiation phase value. Each unit cell of the metasurface array functions as a pixel for the multi-level holography whose local radiation of phase and amplitude can be accurately manipulated. This method provides a passive way for a single holographic image where the required pattern is fixed by the lithography. Here we propose that the MEMS metadevice provides the possibility to realize a real-time rewritable multi-level phase-only holographic display using the actively controlled radiation phase value operating in the underdamped region. 
a

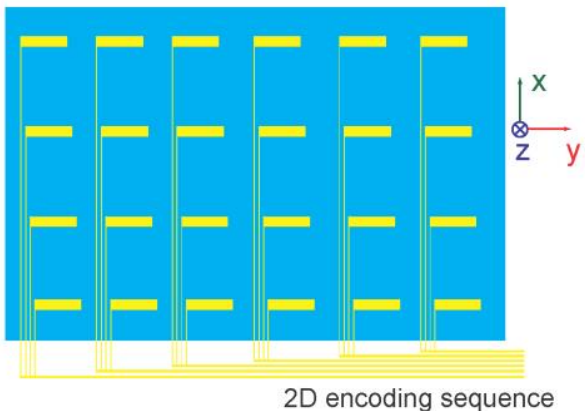

C
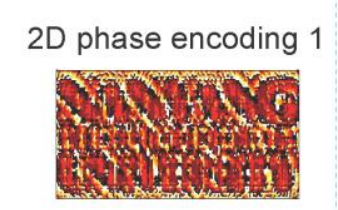

Holographic image 1 b

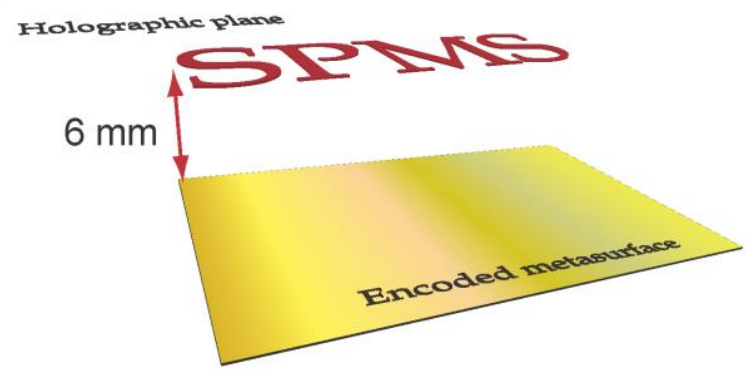

2D phase encoding 2

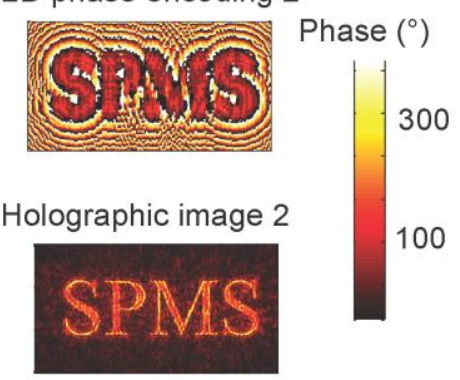

Figure 7-8. Proposal of a real-time holographic display. (a) Schematic illustration for the local control of each unit cell in the MEMS metadevice array. (b) Method to retrieve the phase distribution of the images. (c) Demonstration of the real-time display by actively controlled local radiation phase using MEMS metadevice.

We have proposed one of the possible schemes to realize the real-time holographic display as schematically illustrated in Figure 7-8(a) where each unit cell (pixel) is applied with the corresponding encoding voltages. The encoding sequence is calculated from the images that we want to display at a designated plane using the Rayleigh-Sommerfeld diffraction theory. As shown in Figure 7-8(b), a virtual object is assumed to be at the plane of $d=6$ $\mathrm{mm}$ above the metadevice surface. For simplicity, we consider the words "NANYANG TECHNOLOGICAL UNIVERSITY" and "SPMS" as the holographic images for display. Employing the Rayleigh-Sommerfeld diffraction theory, we can calculate the required phase distributions of each image at the metadevice surface by: 
$U\left(r_{0}\right)=\iint_{\Sigma} U\left(r_{1}\right) \frac{1}{i \lambda} \cos \left\langle n, r_{01}\right\rangle \frac{\exp \left(i k r_{01}\right)}{r_{01}} d s$

Here, $U\left(r_{0}\right)$ and $U\left(r_{1}\right)$ are the electric fields at points $R_{0}$ on the metadevice surface and $R_{l}$ on the image plane, respectively; $\Sigma$ represents the holographic plane area; vacuum wavelength is $\lambda ; \mathbf{n}$ is the normal vector of the holographic plane; $r_{01}$ represents the distance between points $R_{0}$ and $R_{l}$; and $\cos <\mathbf{n}, r_{01}>$ represents the inclination factor. After calculation of the field distributions, linear partition method is required to discretize the object into grids for the metadevice unit cells, and thus superposition of point sources is used instead of integral. For the object, we assume the uniformity of amplitude and phase distributions with $U\left(r_{1}\right)=A$, and so the electric field distributions on the metadevice surface is described as:

$U\left(r_{0}\right)=\frac{A d}{i \lambda} \iint_{\Sigma} \frac{\exp \left(i k r_{01}\right)}{r_{01}^{2}} d s$

We calculated a discrete six-level phase distribution of the electric field on the metadevice surface through the algorithm of normalization and discretization. When the corresponding 2D encoding sequence is applied on the metadevice, the holographic images will be displayed at the designated plane by a plane terahertz wave illumination based on the reversibility of light propagation as illustrated in Figure 7-8(b) that is calculated based on the equation:

$U^{\prime}\left(r_{1}\right)=\iint U\left(r_{0}\right) \frac{d}{i \lambda} \frac{\exp \left(-i k r_{01}\right)}{r_{01}^{2}} d s$

Here the electric field distribution at point $R_{I}$ on the holographic plane is $U^{\prime}\left(r_{1}\right)$ and the discretized field at $R_{0}$ on the metadevice surface is $U\left(r_{0}\right)$. 
We then theoretically verified the real-time holographic display using the proposed scheme of MEMS metadevice. As shown in Figure 7-8(c) where the calculated six-level phase distributions and the corresponding holographic images are presented, the phase-only MEMS real-time holographic display is demonstrated by displaying the words "NANYANG TECHNOLOGICAL UNIVERSITY" and "SPMS" through adjusting the 2D encoding sequences. The images are in good quality despite of the randomly distributed intensity across the surface of the 2D image. The overall efficiency of the holographic images are larger than $25 \%$ that is still possible to be improved higher than $80 \%$ by optimizing the design in reflection mode. ${ }^{22}$ Although the possibility of the real-time holographic display has been theoretically demonstrated here, some issues related to the MEMS techniques need to be addressed before the real applications: 1. the MEMS metadevice is required to be in a large area in order to include enough information for a better resolution of images, however, it is difficult to guarantee the uniformity of all the unit cells in a large area MEMS array; 2. A slight deviation of the cantilever angle strongly affects the radiation phase response, and it is still not possible to guarantee the accurate control of all the unit cells; 3. simultaneous encoding of both amplitude and phase is not applicable using the proposed mechanism by using the MEMS scheme for real-time holographic display, and more sophisticated supercells would be required for more complex encoding requirements. 


\subsection{Conclusion}

In this chapter, we introduced the MEMS technique and integrated with a metadevice in order to discuss the active control of the functionalities by electrostatic force through applying external voltage. The meta-insulator-metal structure is used in all the discussions in this chapter operating in reflection mode. First, we experimentally demonstrate the active switch of the system operation state in the MIM metadevice from the underdamped to overdamped region. When the metadevice operates in the underdamped region, a $360^{\circ}$ phase span together with a large shift of resonance frequency by regulating the suspended cantilevers provide the possibilities of actively controlled optical devices: 1. active polarization control by universal control in the whole array. Using the anisotropic unit cell, the anisotropy of the MEMS metadevice is dynamically modulated at varied voltages, and thus the phase difference of output light is actively controlled that enables different desired polarization states. 2. Dynamic wavefront deflection by local control in each column of the array. The MEMS technique provides the freedom of local control in the column of the array that enables an actively encoded 1D phase gradient by applying the pre-calculated voltage sequence. 3. Real-time holographic display by local control in each single unit cell. The MEMS metadevice also provides the possibility of local control in each unit cell as an imaging pixel. Through $2 \mathrm{D}$ encoding in each unit cell, the possibility to realize the realtime holographic display is theoretically demonstrated. The MEMS metadevice could enable the customized functionalities by the local encoding in the entire array that would push the frontiers of the photonic technologies. Although some limitations exist for the realworld applications of the MEMS metadevice, especially for the more complex 2D control, 
our discussion provides an approach for dynamic applications with more sophisticated and reliable techniques being developed. Here in this chapter, we mainly focus on proposing the ideas for potential applications through active control of local response in column or single unit cell in the MEMS metadevice, and more experimental works are in progress for the realization of dynamic multifunctional photonics devices. 


\section{Chapter 8: Outlooks}

The passive metamaterials have been studied for 15 years, and with the developments in metamaterial community, diverse of subdisciplines emerge by combining metamaterials with different research fields. The studies presented in this thesis include both the fundamentals and applications: 1 . the symmetry is the basis of diverse of disciplines. The study and understanding of the polarizing effects from the symmetry of unit cell convey numerous valuable information for the interpretation of behaviors in natural crystals and provide further insight into the intrinsic properties of metamaterials; 2. near-field coupling also exists in lots of systems that sometimes dominates the system performance. Detailed discussion of near-field coupling in metamaterials provides another pathway to investigate the polarizing effects in a more complex system; 3. broadband operation of a flexible quarter wave plate is proposed for a broadband terahertz system that would be the next generation polarizing device for compact optical systems and industrial applications; 4. dynamic MEMS metadevice provides an approach for active control of functionalities that may enable the multifunctional and programmable metadevices. All of these studies could be extended in diverse of subdirections that would be the next step of investigations:

1. From the aspect of symmetry, the collective optical properties of the uniform metamaterial array are determined by the geometric configuration of the unit cell. For a more complex case where the metamaterial array consists of meta-molecules (consisting of several meta-atoms), spatial configuration of these meta-atoms in the meta-molecule will also affect the optical properties of the metamaterial. The higher 
order symmetry inside the meta-molecule would enable many promising effects such as reducing the radiative loss and inducing the exclusive toroidal moments.

2. Near-field coupling effects have been studied mainly on the in-plane configurations in this thesis, and the out-of-plane coupling effects would also be of great interest for lots of applications. One of the promising fields is the longitudinal coupling between the multilayer metasurfaces, such as in designing the broadband quarter wave plate in this thesis, which would provide more guidance on designing high-performance polarizing or functional devices. Another uncultivated field is the coupling effect in a hybrid system such as MEMS systems and integrated metamaterials with dynamic materials.

3. The theory of dispersion compensation using two layers of metasurfaces would be applied in designing the broadband optical devices with different functionalities, such as a modulator, filter and beam splitter.

Many more investigations related to metamaterials and polarization optics will be studied and discussed in the future including:

1. Due to the strong local field enhancement of metamaterial at resonance while interacting with the electromagnetic waves, the metamaterial would be a promising platform as an ultrasensitive sensor. Slight external disturbance gives rise to an intense modulation of properties of the metamaterial, which could be reflected in the modulation of output polarization states. They are easily to be captured and expressed numerically, therefore, an ultrasensitive sensor based on the modulation of polarization 
ellipticity would be promising for applications in display devices.

2. The resonators of current metamaterials are usually made of metals and the intrinsic losses from the metal decrease the efficiency. All-dielectric metamaterials using Mie scattering avoid the ohmic losses from metals, and provide the high-efficiency metadevices, which are made of dielectric resonators excluding metals. The polarizing devices designed by all-dielectric metamaterials would be promising in providing better performance.

3. Another approach to reduce the intrinsic losses is by using superconductors. Combining metamaterials with superconductors, many interesting phenomena are expected, such as the shift of the critical temperature of the superconductor. Lots of extraordinary resonant properties due to superconductivity of materials are still unexplored, and metamaterials would be an excellent platform for the deeper investigation.

4. The MEMS based metadevice provides the freedom to control the response of each unit cell separately for applications ranging from polarizing devices to flat lenses and rewritable holographic display. However, mechanical modulation of the cantilevers has certain limitations, such as the nonuniform modulation of each unit cell in a large area array and the low modulation speed around $\mathrm{kHz}$. By integrating dynamic media to the metamaterial, ultrafast and uniform modulation of the resonant features are accessible such as by using two-dimentional materials (graphene, $\mathrm{MoS}_{2}$ ) or phase change materials 
$\left(\mathrm{VO}_{2}, \mathrm{GST}\right)$ through bias or optical pump.

5. In addition to the modulation of metamaterial response by dynamic media, we can also investigate the enhancement of material properties due to the presence metamaterials where metamaterials provide an extraordinary local field enhancement, such as the enhancement of material anisotropy, nonlinearity and luminescence efficiency, and so on. 


\section{Appendix: Publications}

\section{$\diamond$ Journal publications during PhD candidature}

1. L. Cong, N. Xu, W. Zhang, and R. Singh, "Polarization Control in Terahertz Metasurfaces with the Lowest Order Rotational Symmetry," Adv. Opt. Mater. 3, 1176 (2015).

2. L. Cong, S. Tan, R. Yahiaoui, F. Yan, W. Zhang, and R. Singh, "Experimental demonstration of ultrasensitive sensing with terahertz metamaterial absorbers: A comparison with the metasurfaces," Appl. Phys. Lett. 106, 031107 (2015). (Selected as the must-read findings on terahertz devices by AIP) Link: http://aip-info.org/1XPS3OQX8-25IQEZQ494/cr.aspx.

3. L. Cong, M. Manjappa, N. Xu, I. Al-Naib, W. Zhang, and R. Singh, "Fano Resonances in Terahertz Metasurfaces: A Figure of Merit Optimization," Adv. Opt. Mater. 3, 1537 (2015). (Highlighted as the top 5 download papers in Sep. by Wiley) Link: http://www.materialsviews.com/advanced-optical-materials-top-5-sept-2015/.

4. L. Cong, N. Xu, J. Han, W. Zhang, and R. Singh, "A Tunable Dispersion-Free Terahertz Metadevice with Pancharatnam-Berry-Phase-Enabled Modulation and Polarization Control," Adv. Mater. 27, 6630 (2015).

5. L. Cong, N. Xu, D. R. Chowdhury, M. Manjappa, C. Rockstuhl, W. Zhang, and R. Singh, "Nonradiative and Radiative Resonances in Coupled Metamolecules," Adv. Opt. Mater. 4, 252-258 (2016). 
6. L. Cong, Y. K. Srivastava, and R. Singh, "Inter and intra-metamolecular interaction enabled broadband high-efficiency polarization control in metasurfaces," Appl. Phys. Lett. 108, 011110 (2016).

7. L. Cong, Y. Srivastava, R. Singh, "Near-field inductive coupling induced polarization control in metasurfaces," Adv. Opt. Mater.4, 848 (2016).

8. L. Cong, P. Pitchappa, Y. Wu, L. Ke, C. Lee, N. Singh, H. Yang, and R. Singh, “Active multifunctional MEMS metadevices: Applications in polarization control, wavefront deflection and holograms," Adv. Opt. Mater. 5, 1600716 (2017).

9. L. Cong, P. Pitchappa, C. Lee and R. Singh, "Active Phase Transition via Loss Engineering in a Terahertz MEMS Metamaterial," Adv. Mater., 1700733 (2017).

10. L. Cong, Y. K. Srivastava, A. Solanki, T. C. Sum and R. Singh, "Perovskite as a Platform for Active Flexible Metaphotonic Devices," ACS Photonics (2017).

\section{$\diamond$ Collaborative publications}

1. M. Lawrence, N. Xu, X. Zhang, L. Cong, J. Han, W. Zhang, and S. Zhang, "Manifestation of P T Symmetry Breaking in Polarization Space with Terahertz Metasurfaces," Phys. Rev. Lett. 113, 093901 (2014).

2. R. Singh, I. Al-Naib, D. R. Chowdhury, L. Cong, C. Rockstuhl, and W. Zhang, "Probing the transition from an uncoupled to a strong near-field coupled regime between bright and dark mode resonators in metasurfaces," Appl. Phys. Lett. 105, 081108 (2014).

3. R. Singh, W. Cao, I. Al-Naib, L. Cong, W. Withayachumnankul, and W. Zhang, "Ultrasensitive terahertz sensing with high-Q Fano resonances in metasurfaces," Appl. 
Phys. Lett. 105, 171101 (2014). (Selected as the must-read findings on terahertz devices by AIP) Link: http://aip-info.org/1XPS-3OQX8-25IQEZQ494/cr.aspx.

4. S. Han, R. Singh, L. Cong, and H. Yang, "Engineering the fano resonance and electromagnetically induced transparency in near-field coupled bright and dark metamaterial," J. of Phys. D: Appl. Phys. 48, 035104 (2015).

5. M. Manjappa, S.-Y. Chiam, L. Cong, A. A. Bettiol, W. Zhang, and R. Singh, "Tailoring the slow light behavior in terahertz metasurfaces," Appl. Phys. Lett. 106, 181101 (2015).

6. R. Yahiaoui, S. Tan, L. Cong, R. Singh, F. Yan, and W. Zhang, "Multispectral terahertz sensing with highly flexible ultrathin metamaterial absorber," J. of Appl. Phys. 118, 083103 (2015).

7. Y. K. Srivastava, M. Manjappa, L. Cong, W. Cao, I. Al-Naib, W. Zhang, and R. Singh, "Ultrahigh-Q Fano Resonances in Terahertz Metasurfaces: Strong Influence of Metallic Conductivity at Extremely Low Asymmetry," Adv. Opt. Mater. 4, 457-463 (2016).

8. P. Pitchappa, C. P. Ho, L. Cong, R. Singh, N. Singh, and C. Lee, "Reconfigurable Digital Metamaterial for Dynamic Switching of Terahertz Anisotropy," Adv. Opt. Mater. 4, 391398 (2016).

9. S. Han, L. Cong, H. Lin, B. Xiao, H. Yang, and R. Singh, "Tunable electromagnetically induced transparency in coupled three-dimensional split-ring-resonator metamaterials," Scientific reports 6, 20801 (2016).

10. S. Han, L. Cong, F. Gao, R. Singh and H. Yang, "Observation of Fano resonance and classical analog of electromagnetically induced transparency in toroidal metamaterials," Annalen der Physik, 528, 352-357 (2016). 
11. M. Gupta, V. Savinov, N. Xu, L. Cong, G. Dayal, S. Wang, W. Zhang, N. I. Zheludev, and R. Singh, "Sharp Toroidal Resonances in Planar Terahertz Metasurfaces," Adv. Mater. 28, 8206-8211 (2016).

12. Q. Li, ${ }^{*}$ L. Cong, ${ }^{*}$ R. Singh, N. Xu, W. Cao, X. Zhang, Z. Tian, L. Du, J. Han and W. Zhang, "Monolayer graphene sensing enabled by the strong Fano-resonant metasurface," Nanoscale 8, 17278-17284 (2016).

13. M. Manjappa, ${ }^{*}$ Y. K. Srivastava, ${ }^{*}$ L. Cong, ${ }^{*}$ I. Al-Naib, and R. Singh, "Active Photoswitching of Sharp Fano Resonances in THz Metadevices," Adv. Mater. 29, 1603355 (2017).

\section{$\diamond$ Journal publications for reference}

1. Y. Yang, R. Huang, L. Cong, Z. Zhu, J. Gu, Z. Tian, R. Singh, S. Zhang, J. Han, and W. Zhang, "Modulating the fundamental inductive-capacitive resonance in asymmetric double-split ring terahertz metamaterials," Appl. Phys. Lett. 98, 121114 (2011).

2. L. Cong, W. Cao, Z. Tian, J. Gu, J. Han, and W. Zhang, "Manipulating polarization states of terahertz radiation using metamaterials," New J. of Phys. 14, 115013 (2012).

3. L. Cong, W. Cao, X. Zhang, Z. Tian, J. Gu, R. Singh, J. Han, and W. Zhang, "A perfect metamaterial polarization rotator," Appl. Phys. Lett. 103, 171107 (2013).

4. W. Song, Z. Wang, S. Wang, M. He, J. Han, and L. Cong, "Active modulation of refractive index by stress in the terahertz frequency range," Appl. Optics 52, 6364 (2013).

5. L. Cong, N. Xu, J. Gu, R. Singh, J. Han, and W. Zhang, "Highly flexible broadband 
terahertz metamaterial quarter - wave plate," Laser \& Photonics Rev. 8, 626 (2014). (Highlighted as inside cover page).

6. W. Song, Z. Wang, S. Wang, M. He, J. Han, L. Cong, and Y. Deng, "Experimental verification of the uniaxial stress-optic law in the terahertz frequency regime," Optics and Lasers in Engineering 52, 174 (2014).

7. L. Cong, J. Gu, Z. Tian, R. Singh, J. Han, and W. Zhang, "Manipulating the polarization of terahertz waves with metamaterial devices," Journal of Molecular and Engineering Materials 2, 1440008 (2014). 


\section{References:}

1. Y.-S. Lee, Principles of terahertz science and technology. (Springer Science \& Business Media, 2009).

2. M. Tonouchi, Nature photonics 1 (2), 97-105 (2007).

3. N. Liu, M. Mesch, T. Weiss, M. Hentschel and H. Giessen, Nano Lett. 10 (7), 2342-2348 (2010).

4. C. M. Watts, X. Liu and W. J. Padilla, Adv. Mater. 24 (23), OP98-120, OP181 (2012).

5. N. Landy, S. Sajuyigbe, J. Mock, D. Smith and W. Padilla, Phys. Rev. Lett. 100 (20) (2008).

6. L. Martin-Moreno, F. Garcia-Vidal, H. Lezec, K. Pellerin, T. Thio, J. Pendry and T. Ebbesen, Phys. Rev. Lett. 86 (6), 1114 (2001).

7. N. Yu, P. Genevet, M. A. Kats, F. Aieta, J. P. Tetienne, F. Capasso and Z. Gaburro, Science 334 (6054), 333-337 (2011).

8. D. R. Smith, J. B. Pendry and M. C. Wiltshire, Science 305 (5685), 788-792 (2004).

9. T. J. Yen, W. J. Padilla, N. Fang, D. C. Vier, D. R. Smith, J. B. Pendry, D. N. Basov and X. Zhang, Science 303 (5663), 1494-1496 (2004).

10. J. Pendry, Science 306 (5700), 1353-1355 (2004).

11. J. K. Gansel, M. Thiel, M. S. Rill, M. Decker, K. Bade, V. Saile, G. von Freymann, S. Linden and M. Wegener, Science 325 (5947), 1513-1515 (2009).

12. C. Wu, A. B. Khanikaev, R. Adato, N. Arju, A. A. Yanik, H. Altug and G. Shvets, Nature materials 11 (1), 69-75 (2012).

13. A. Kabashin, P. Evans, S. Pastkovsky, W. Hendren, G. Wurtz, R. Atkinson, R. Pollard, V. Podolskiy and A. Zayats, Nature materials 8 (11), 867-871 (2009).

14. N. Liu, T. Weiss, M. Mesch, L. Langguth, U. Eigenthaler, M. Hirscher, C. Sönnichsen and H. Giessen, Nano Lett. 10 (4), 1103-1107 (2009).

15. N. Yu, Q. J. Wang, M. A. Kats, J. A. Fan, S. P. Khanna, L. Li, A. G. Davies, E. H. Linfield and F. Capasso, Nat Mater 9 (9), 730-735 (2010).

16. N. Yu and F. Capasso, Nat Mater 13 (2), 139-150 (2014).

17. V. G. Veselago, Soviet physics uspekhi 10 (4), 509 (1968).

18. J. B. Pendry, Contemporary Physics 45 (3), 191-202 (2004).

19. Q. Wang, E. T. F. Rogers, B. Gholipour, C.-M. Wang, G. Yuan, J. Teng and N. I. Zheludev, Nature Photonics 10 (1), 60-65 (2016).

20. M. Khorasaninejad, W. T. Chen, R. C. Devlin, J. Oh, A. Y. Zhu and F. Capasso, Science 352 (6290), 1190-1194 (2016).

21. L. Huang, X. Chen, H. Mühlenbernd, H. Zhang, S. Chen, B. Bai, Q. Tan, G. Jin, K.-W. Cheah and C.-W. Qiu, Nat Commun 4 (2013).

22. G. Zheng, H. Mühlenbernd, M. Kenney, G. Li, T. Zentgraf and S. Zhang, Nat Nano 10 (4), 308-312 (2015).

23. X. Ni, Z. J. Wong, M. Mrejen, Y. Wang and X. Zhang, Science 349 (6254), 1310-1314 (2015).

24. L.-H. Gao, Q. Cheng, J. Yang, S.-J. Ma, J. Zhao, S. Liu, H.-B. Chen, Q. He, W.-X. Jiang, 
H.-F. Ma, Q.-Y. Wen, L.-J. Liang, B.-B. Jin, W.-W. Liu, L. Zhou, J.-Q. Yao, P.-H. Wu and T.-J. Cui, Light Sci Appl 4, e324 (2015).

25. T. J. Cui, M. Q. Qi, X. Wan, J. Zhao and Q. Cheng, Light: Science \& Applications 3 (10), e218 (2014).

26. S. Zhang, Y.-S. Park, J. Li, X. Lu, W. Zhang and X. Zhang, Phys. Rev. Lett. 102 (2) (2009).

27. Y. Ye and S. He, Appl. Phys. Lett. 96 (20), 203501 (2010).

28. E. Plum, X. X. Liu, V. Fedotov, Y. Chen, D. Tsai and N. Zheludev, Phys. Rev. Lett. 102 (11) (2009).

29. J. Y. Chin, T. Steinle, T. Wehlus, D. Dregely, T. Weiss, V. I. Belotelov, B. Stritzker and H. Giessen, Nat Commun 4, 1599 (2013).

30. N. Koumura, R. W. Zijlstra, R. A. van Delden, N. Harada and B. L. Feringa, Nature 401 (6749), 152-155 (1999).

31. J. J. de Jong, L. N. Lucas, R. M. Kellogg, J. H. van Esch and B. L. Feringa, Science 304 (5668), 278-281 (2004).

32. S. Zhang, J. Zhou, Y. S. Park, J. Rho, R. Singh, S. Nam, A. K. Azad, H. T. Chen, X. Yin, A. J. Taylor and X. Zhang, Nat Commun 3, 942 (2012).

33. T. Kan, A. Isozaki, N. Kanda, N. Nemoto, K. Konishi, H. Takahashi, M. KuwataGonokami, K. Matsumoto and I. Shimoyama, Nat Commun 6 (2015).

34. V. Fedotov, P. Mladyonov, S. Prosvirnin, A. Rogacheva, Y. Chen and N. Zheludev, Phys. Rev. Lett. 97 (16) (2006).

35. R. Singh, E. Plum, C. Menzel, C. Rockstuhl, A. Azad, R. Cheville, F. Lederer, W. Zhang and N. Zheludev, Physical Review B 80 (15) (2009).

36. C. Menzel, C. Helgert, C. Rockstuhl, E.-B. Kley, A. Tünnermann, T. Pertsch and F. Lederer, Phys. Rev. Lett. 104 (25), 253902 (2010).

37. J. Kyoung, E. Y. Jang, M. D. Lima, H. R. Park, R. O. Robles, X. Lepro, Y. H. Kim, R. H. Baughman and D. S. Kim, Nano Lett. 11 (10), 4227-4231 (2011).

38. A. Arbabi, Y. Horie, M. Bagheri and A. Faraon, Nat Nano 10 (11), 937-943 (2015).

39. D. Goldstein and D. H. Goldstein, Polarized Light, revised and expanded. (CRC Press, 2011).

40. M. Born and E. Wolf, Principles of optics: electromagnetic theory of propagation, interference and diffraction of light. (CUP Archive, 2000).

41. G. G. Stokes, Transactions of the Cambridge Philosophical Society 9, 399 (1851).

42. R. C. Jones, JOSA 31 (7), 488-493 (1941).

43. H. Poincaré, Théorie mathématique de la lumiere. (Gauthier Villars, 1892).

44. W. A. Shurcliff, Cambridge, Mass.: Harvard University Press,| c1966 1 (1966).

45. E. W. Weisstein, Stereographic Projection. (Wolfram Mathworld, 2002).

46. A. Zee, Fearful symmetry: the search for beauty in modern physics. (Princeton University Press, 2015).

47. K. Mainzer, Symmetry and complexity: The spirit and beauty of nonlinear science. (World Scientific, 2005).

48. N. P. Huck, W. F. Jager, B. de Lange and B. L. Feringa, Science 273 (5282), 1686-1688 (1996).

49. L. Ge and A. D. Stone, Physical Review X 4 (3), 031011 (2014). 
50. H. Hodaei, M.-A. Miri, M. Heinrich, D. N. Christodoulides and M. Khajavikhan, Science 346 (6212), 975-978 (2014).

51. M. Lawrence, N. Xu, X. Zhang, L. Cong, J. Han, W. Zhang and S. Zhang, Phys. Rev. Lett. 113 (9), 093901 (2014).

52. L. Feng, Z. J. Wong, R.-M. Ma, Y. Wang and X. Zhang, Science 346 (6212), 972-975 (2014).

53. D. Smith, J. Pendry and M. Wiltshire, Science 305 (5685), 788-792 (2004).

54. J. B. Pendry, Phys. Rev. Lett. 85 (18), 3966 (2000).

55. M. Khorasaninejad, W. T. Chen, J. Oh and F. Capasso, Nano Lett. (2016).

56. N. Yu and F. Capasso, Nat Mater 13 (2), 139-150 (2014).

57. N. Yu, P. Genevet, M. A. Kats, F. Aieta, J.-P. Tetienne, F. Capasso and Z. Gaburro, Science 334 (6054), 333-337 (2011).

58. S. Zhang, J. Zhou, Y.-S. Park, J. Rho, R. Singh, S. Nam, A. K. Azad, H.-T. Chen, X. Yin and A. J. Taylor, Nat Commun 3, 942 (2012).

59. J. K. Gansel, M. Thiel, M. S. Rill, M. Decker, K. Bade, V. Saile, G. von Freymann, S. Linden and M. Wegener, Science 325 (5947), 1513-1515 (2009).

60. R. Singh, E. Plum, C. Menzel, C. Rockstuhl, A. Azad, R. Cheville, F. Lederer, W. Zhang and N. Zheludev, Physical Review B 80 (15), 153104 (2009).

61. V. Fedotov, P. Mladyonov, S. Prosvirnin, A. Rogacheva, Y. Chen and N. Zheludev, Phys. Rev. Lett. 97 (16), 167401 (2006).

62. K. Konishi, M. Nomura, N. Kumagai, S. Iwamoto, Y. Arakawa and M. KuwataGonokami, Phys. Rev. Lett. 106 (5), 057402 (2011).

63. N. Kanda, K. Konishi and M. Kuwata-Gonokami, Opt. Lett. 34 (19), 3000-3002 (2009).

64. K. Song, X. Zhao, Y. Liu, Q. Fu and C. Luo, Appl. Phys. Lett. 103 (10), 101908 (2013). 65. E. Plum, X.-X. Liu, V. Fedotov, Y. Chen, D. Tsai and N. Zheludev, Phys. Rev. Lett. 102 (11), 113902 (2009).

66. Y. Zhao, M. Belkin and A. Alù, Nat Commun 3, 870 (2012).

67. L. Cong, N. Xu, W. Zhang and R. Singh, Advanced Optical Materials 3 (9), 1176-1183 (2015).

68. X. Ding, F. Monticone, K. Zhang, L. Zhang, D. Gao, S. N. Burokur, A. de Lustrac, Q. Wu, C. W. Qiu and A. Alù, Adv. Mater. (2014).

69. F. Monticone, N. M. Estakhri and A. Alù, Phys. Rev. Lett. 110 (20), 203903 (2013).

70. C. Menzel, C. Rockstuhl and F. Lederer, Phys. Rev. A 82 (5), 053811 (2010).

71. M. S. Rill, C. Plet, M. Thiel, I. Staude, G. Von Freymann, S. Linden and M. Wegener, Nat Mater 7 (7), 543-546 (2008).

72. N. Liu, H. Liu, S. Zhu and H. Giessen, Nature Photonics 3 (3), 157-162 (2009).

73. L. Cong, W. Cao, X. Zhang, Z. Tian, J. Gu, R. Singh, J. Han and W. Zhang, Appl. Phys. Lett. 103 (17), 171107 (2013).

74. L. Cong, N. Xu, J. Gu, R. Singh, J. Han and W. Zhang, Laser \& Photonics Reviews 8 (4), 626-632 (2014).

75. L. Cong, N. Xu, J. Han, W. Zhang and R. Singh, Adv. Mater. 27 (42), 6630-6636 (2015).

76. M. Scheller, C. Jördens and M. Koch, Opt. Express 18 (10), 10137-10142 (2010).

77. B. Päivänranta, N. Passilly, J. Pietarinen, P. Laakkonen, M. Kuittinen and J. Tervo, Opt. Express 16 (21), 16334-16342 (2008). 
78. B. Scherger, M. Scheller, N. Vieweg, S. T. Cundiff and M. Koch, Opt. Express 19 (25), 24884-24889 (2011).

79. P. Weis, O. Paul, C. Imhof, R. Beigang and M. Rahm, Appl. Phys. Lett. 95 (17), 171104 (2009).

80. V. E. Ferry, M. A. Verschuuren, H. B. Li, E. Verhagen, R. J. Walters, R. E. Schropp, H. A. Atwater and A. Polman, Opt. Express 18 (102), A237-A245 (2010).

81. T. Clausnitzer, J. Limpert, K. Zöllner, H. Zellmer, H.-J. Fuchs, E.-B. Kley, A. Tünnermann, M. Jupe and D. Ristau, Appl. Opt. 42 (34), 6934-6938 (2003).

82. J. Feng, C. Zhou, B. Wang, J. Zheng, W. Jia, H. Cao and P. Lv, Appl. Opt. 47 (35), 66386643 (2008).

83. M. Khorasaninejad, W. Zhu and K. Crozier, Optica 2 (4), 376-382 (2015).

84. J. K. Hyun, J. Park, E. Kim, L. J. Lauhon and S. Jeon, Advanced Optical Materials 2 (12), 1213-1220 (2014).

85. R. Singh, I. Al-Naib, D. R. Chowdhury, L. Cong, C. Rockstuhl and W. Zhang, Appl. Phys. Lett. 105 (8), 081108 (2014).

86. S. Zhang, D. A. Genov, Y. Wang, M. Liu and X. Zhang, Phys. Rev. Lett. 101 (4), 047401 (2008).

87. N. Liu, L. Langguth, T. Weiss, J. Kastel, M. Fleischhauer, T. Pfau and H. Giessen, Nat Mater 8 (9), 758-762 (2009).

88. N. Liu and H. Giessen, Angew. Chem. Int. Ed. 49 (51), 9838-9852 (2010).

89. B. Abasahl, S. Dutta-Gupta, C. Santschi and O. J. Martin, Nano Lett. 13 (9), 4575-4579 (2013).

90. I. Sersic, M. Frimmer, E. Verhagen and A. F. Koenderink, Phys. Rev. Lett. 103 (21) (2009).

91. N. Liu, S. Kaiser and H. Giessen, Adv. Mater. 20 (23), 4521-4525 (2008).

92. P. Q. Liu, I. J. Luxmoore, S. A. Mikhailov, N. A. Savostianova, F. Valmorra, J. Faist and G. R. Nash, Nat Commun 6 (2015).

93. Y. Yang, Kravchenko, II, D. P. Briggs and J. Valentine, Nat Commun 5, 5753 (2014).

94. J. Gu, R. Singh, X. Liu, X. Zhang, Y. Ma, S. Zhang, S. A. Maier, Z. Tian, A. K. Azad and H.-T. Chen, Nature communications 3, 1151 (2012).

95. S.-Y. Chiam, R. Singh, C. Rockstuhl, F. Lederer, W. Zhang and A. A. Bettiol, Physical Review B 80 (15), 153103 (2009).

96. R. Singh, C. Rockstuhl, F. Lederer and W. Zhang, Physical Review B 79 (8) (2009).

97. L. Cong, N. Xu, D. R. Chowdhury, M. Manjappa, C. Rockstuhl, W. Zhang and R. Singh, Advanced Optical Materials 4 (2), 252-258 (2016).

98. X. Zhang, N. Xu, K. Qu, Z. Tian, R. Singh, J. Han, G. S. Agarwal and W. Zhang, Scientific reports 5 (2015).

99. B. Gallinet and O. J. Martin, Physical Review B 83 (23), 235427 (2011).

100. Y. S. Joe, A. M. Satanin and C. S. Kim, Phys. Scr. 74 (2), 259 (2006).

101. V. Fedotov, M. Rose, S. Prosvirnin, N. Papasimakis and N. Zheludev, Phys. Rev. Lett. 99 (14), 147401 (2007).

102. L. Cong, M. Manjappa, N. Xu, I. Al-Naib, W. Zhang and R. Singh, Advanced Optical Materials 3 (11), 1537-1543 (2015).

103. B. Luk'yanchuk, N. I. Zheludev, S. A. Maier, N. J. Halas, P. Nordlander, H. Giessen 
and C. T. Chong, Nature materials 9 (9), 707-715 (2010).

104. T. Kaelberer, V. A. Fedotov, N. Papasimakis, D. P. Tsai and N. I. Zheludev, Science 330 (6010), 1510-1512 (2010).

105. S. Linden, C. Enkrich, M. Wegener, J. Zhou, T. Koschny and C. M. Soukoulis, Science 306 (5700), 1351-1353 (2004).

106. L. Cong, Y. K. Srivastava and R. Singh, Appl. Phys. Lett. 108 (1), 011110 (2016).

107. L. Cong, Y. K. Srivastava and R. Singh, Advanced Optical Materials 4 (6), 848-852 (2016).

108. M. Decker, R. Zhao, C. Soukoulis, S. Linden and M. Wegener, Opt. Lett. 35 (10), 1593-1595 (2010).

109. X. Zhang, Q. Li, W. Cao, W. Yue, J. Gu, Z. Tian, J. Han and W. Zhang, Chinese Optics Letters 9 (11), 110012 (2011).

110. J. D. Baena, J. Bonache, F. Martin, R. M. Sillero, F. Falcone, T. Lopetegi, M. A. Laso, J. Garcia-Garcia, I. Gil and M. F. Portillo, IEEE Trans. Microwave Theory Tech. 53 (4), 1451-1461 (2005).

111. R. Singh, C. Rockstuhl and W. Zhang, Appl. Phys. Lett. 97 (24), 241108 (2010).

112. N. Xu, R. Singh and W. Zhang, J. Appl. Phys. 118 (16), 163102 (2015).

113. V. Fedotov, N. Papasimakis, E. Plum, A. Bitzer, M. Walther, P. Kuo, D. Tsai and N. Zheludev, Phys. Rev. Lett. 104 (22), 223901 (2010).

114. N. I. Zheludev, S. Prosvirnin, N. Papasimakis and V. Fedotov, Nature Photonics 2 (6), 351-354 (2008).

115. L. Cong, S. Tan, R. Yahiaoui, F. Yan, W. Zhang and R. Singh, Appl. Phys. Lett. 106 (3), 031107 (2015).

116. R. Yahiaoui, S. Tan, L. Cong, R. Singh, F. Yan and W. Zhang, J. Appl. Phys. 118 (8), 083103 (2015).

117. R. Singh, W. Cao, I. Al-Naib, L. Cong, W. Withayachumnankul and W. Zhang, Appl. Phys. Lett. 105 (17), 171101 (2014).

118. W. Cao, R. Singh, I. A. Al-Naib, M. He, A. J. Taylor and W. Zhang, Opt. Lett. 37 (16), 3366-3368 (2012).

119. S. Han, L. Cong, F. Gao, R. Singh and H. Yang, Annalen der Physik 528 (5), 352-357 (2016).

120. I. Al-Naib, Y. Yang, M. M. Dignam, W. Zhang and R. Singh, Appl. Phys. Lett. 106 (1), 011102 (2015).

121. Y. K. Srivastava, M. Manjappa, L. Cong, W. Cao, I. Al-Naib, W. Zhang and R. Singh, Advanced Optical Materials 4 (3), 457-463 (2016).

122. Y. Yang, W. Wang, P. Moitra, I. I. Kravchenko, D. P. Briggs and J. Valentine, Nano Lett. 14 (3), 1394-1399 (2014).

123. N. Radwell, R. D. Hawley, J. B. Gotte and S. Franke-Arnold, Nat Commun 7, 10564 (2016).

124. Z. Wei, Y. Cao, Y. Fan, X. Yu and H. Li, Appl. Phys. Lett. 99 (22), 221907 (2011).

125. N. Yu, F. Aieta, P. Genevet, M. A. Kats, Z. Gaburro and F. Capasso, Nano Lett. 12 (12), 6328-6333 (2012).

126. C. Argyropoulos, K. Q. Le, N. Mattiucci, G. D’Aguanno and A. Alù, Physical Review B 87 (20) (2013). 
127. P. Zhu and L. Jay Guo, Appl. Phys. Lett. 101 (24), 241116 (2012).

128. X. Zhang, J. Gu, W. Cao, J. Han, A. Lakhtakia and W. Zhang, Opt. Lett. 37 (5), 906908 (2012).

129. M. Liu, X. Yin, E. Ulin-Avila, B. Geng, T. Zentgraf, L. Ju, F. Wang and X. Zhang, Nature 474 (7349), 64-67 (2011).

130. Q. Li, Z. Tian, X. Zhang, R. Singh, L. Du, J. Gu, J. Han and W. Zhang, Nat Commun $6(2015)$.

131. D. Shin, Y. Urzhumov, Y. Jung, G. Kang, S. Baek, M. Choi, H. Park, K. Kim and D. R. Smith, Nat Commun 3, 1213 (2012).

132. X. Ni, N. K. Emani, A. V. Kildishev, A. Boltasseva and V. M. Shalaev, Science 335 (6067), 427 (2012).

133. F. Ding, Y. Cui, X. Ge, Y. Jin and S. He, Appl. Phys. Lett. 100 (10), 103506 (2012).

134. D. Wen, F. Yue, G. Li, G. Zheng, K. Chan, S. Chen, M. Chen, K. F. Li, P. W. H. Wong, K. W. Cheah, E. Yue Bun Pun, S. Zhang and X. Chen, Nat Commun 6 (2015).

135. X. Ni, A. V. Kildishev and V. M. Shalaev, Nat Commun 4 (2013).

136. Y.-W. Huang, W. T. Chen, W.-Y. Tsai, P. C. Wu, C.-M. Wang, G. Sun and D. P. Tsai, Nano Lett. 15 (5), 3122-3127 (2015).

137. J. Gu, R. Singh, X. Liu, X. Zhang, Y. Ma, S. Zhang, S. A. Maier, Z. Tian, A. K. Azad and H.-T. Chen, Nat Commun 3, 1151 (2012).

138. Z. Miao, Q. Wu, X. Li, Q. He, K. Ding, Z. An, Y. Zhang and L. Zhou, Physical Review X 5 (4), 041027 (2015).

139. C. Qu, S. Ma, J. Hao, M. Qiu, X. Li, S. Xiao, Z. Miao, N. Dai, Q. He and S. Sun, Phys. Rev. Lett. 115 (23), 235503 (2015).

140. Y. Wu, C. La - o - vorakiat, X. Qiu, J. Liu, P. Deorani, K. Banerjee, J. Son, Y. Chen, E. E. Chia and H. Yang, Adv. Mater. 27 (11), 1874-1879 (2015).

141. N. I. Zheludev and Y. S. Kivshar, Nature materials 11 (11), 917-924 (2012).

142. H. Tao, A. C. Strikwerda, K. Fan, W. J. Padilla, X. Zhang and R. D. Averitt, Phys. Rev. Lett. 103 (14), 147401 (2009).

143. F. Ma, Y.-S. Lin, X. Zhang and C. Lee, Light: Science \& Applications 3 (5), e171 (2014).

144. W. M. Zhu, A. Q. Liu, T. Bourouina, D. P. Tsai, J. H. Teng, X. H. Zhang, G. Q. Lo, D. L. Kwong and N. I. Zheludev, Nat Commun 3, 1274 (2012).

145. J. Y. Ou, E. Plum, L. Jiang and N. I. Zheludev, Nano Lett. 11 (5), 2142-2144 (2011).

146. M. Unlu, M. R. Hashemi, C. W. Berry, S. Li, S. H. Yang and M. Jarrahi, Scientific Reports 4, 5708 (2014).

147. N. I. Zheludev and E. Plum, Nature nanotechnology 11 (1), 16-22 (2016).

148. J. Y. Ou, E. Plum, J. Zhang and N. I. Zheludev, Adv. Mater. 28 (4), 729-733 (2016).

149. X. Liu and W. J. Padilla, Adv. Mater. 28 (5), 871-875 (2016).

150. P. Pitchappa, C. P. Ho, L. Cong, R. Singh, N. Singh and C. Lee, Advanced Optical Materials 4 (3), 391-398 (2016).

151. P. Pitchappa, H. Chong Pei, L. Dhakar, Q. You, N. Singh and L. Chengkuo, Microelectromechanical Systems, Journal of 24 (3), 525-527 (2015).

152. H. A. Haus, Waves and fields in optoelectronics. (Prentice Hall, Incorporated, 1984).

153. F. Garcia-Vidal, L. Martin-Moreno and J. Pendry, Journal of optics A: Pure and applied 
optics 7 (2), S97 (2005).

154. X. Ding, F. Monticone, K. Zhang, L. Zhang, D. Gao, S. N. Burokur, A. de Lustrac, Q. Wu, C.-W. Qiu and A. Alù, Adv. Mater. 27 (7), 1195-1200 (2015).

155. Q. Li, Z. Tian, X. Zhang, N. Xu, R. Singh, J. Gu, P. Lv, L.-B. Luo, S. Zhang and J. Han, Carbon 90, 146-153 (2015).

156. X. Zhang, Z. Tian, W. Yue, J. Gu, S. Zhang, J. Han and W. Zhang, Adv. Mater. 25 (33), 4567-4572 (2013).

157. L. Liu, X. Zhang, M. Kenney, X. Su, N. Xu, C. Ouyang, Y. Shi, J. Han, W. Zhang and S. Zhang, Adv. Mater. 26 (29), 5031-5036 (2014).

158. L. Huang, X. Chen, H. Mühlenbernd, H. Zhang, S. Chen, B. Bai, Q. Tan, G. Jin, K.W. Cheah, C.-W. Qiu, J. Li, T. Zentgraf and S. Zhang, Nat Commun 4 (2013). 